\title{
Contrasting approaches to a theory of learning
}

\author{
Timothy D. Johnston \\ North Carolina Division of Mental Health, Research Branch, Dorothea Dix \\ Hospital, Raleigh, N.C. 27611
}

\begin{abstract}
The general process view of learning, which guided research into learning for the first half of this century, has come under attack in recent years from several quarters. One form of criticism has come from proponents of the so-called biological boundaries approach to learning. These theorists have presented a variety of data showing that supposedly general laws of learning may in fact be limited in their applicability to different species and learning tasks, and they argue that the limitations are drawn by the nature of each species, adaptation to the particular requirements of its natural environment. The biological boundaries approach has served an important critical function in the move away from general process learning theory, but it is limited in its ability to provide an alternative to the general process approach. In particular, the biological boundaries approach lacks generality, it is in some respects subservient to the general process tradition, and its ecological content is in too many cases limited to ex post facto adaptive explanations of learning skills. A contrasting, ecological approach to learning, which can provide a true alternative to general process theory, is presented. The ecological approach begins by providing an ecological task description for naturally occurring instances of learning; this step answers the question: What does this animal learn to do? The next step is an analysis of the means by which learning occurs in the course of development, answering the question : How does the animal learn to do this? On the basis of such analyses, local principles of adaptation are formulated to account for the learning abilities of individual species. More global principles are sought by generalization among these local principles and may form the basis for a general ecological theory of learning.
\end{abstract}

Keywords: adaptation; behavioral biology; behaviorism; ecology of learning; learning theory; species-typical behavior

During the first half of this century, psychologists invested an enormous effort in the search for a general theory of learning. Underlying that effort was the belief that all forms of learning could be explained in terms of some fairly small set of general principles. A variety of such principles was proposed, and although their respective merits were widely debated, there was no serious disagreement with the view that some set of general principles existed that would eventually explain all of the phenomena of learning. The general learning theorists of that era held not only that there is a set of general principles applicable to all learning, but also that all examples of learning are manifestations of an underlying general process. That is, they believed that whenever animals learn, not only can we always account for what they do in terms of the same set of principles, but we will also find that they are doing the same sorts of things (e.g. forming stimulusresponse associations).

In recent years, a number of authors (e.g. Seligman 1970; Bolles 1970; Rozin \& Kalat 1971; Shettleworth 1972; Hinde 1973) have taken issue with the general process approach to the study of learning, arguing that many instances of learning provide evidence of rather specialized processes - adaptations to the specific demands of the environments inhabited by different animals. This view, which is discussed in more detail below, has been dubbed the "biological boundaries approach" to the study of learning. Its proponents have provided a number of important criticisms of the general process tradition, but it will be argued in a later section that their contribution is limited in the extent to which it can offer an alternative to that tradition. In particular, the biological boundaries approach has, in many of its formulations, denied the possibility of general principles of learning with the same vigor that it has denied the existence of general processes. Yet the search for general principles must surely be one of the most important aspects of any scientific endeavour. Any denial of their existence in a particular field of inquiry should be accepted only after all possible alternatives have been explored.

This paper discusses one approach to the study of learning that may be a viable alternative to both the general process tradition and the newer biological boundaries approach. It is an ecological approach to the study of learning - one that seeks its general principles in the relationships between animals and their natural environments, rather than in the characteristics of animals alone. The ecological approach will be contrasted with both the biological boundaries approach, from which it differs in the importance attached to the search for general principles of learning, and the general process tradition, from which it differs in the importance attached to ecological considerations in the study of learning. A more detailed 
discussion of the theory that underlies the ecological approach has been published elsewhere (Johnston \& Turvey 1980).

\section{Some terms and distinctions}

A few terms should be defined at the outset. The term "environment" will be used to refer to those aspects of the physical, biological, and social world with which an animal interacts (Mason \& Langenheim 1957), rather than the location, or type of location, that it inhabits. Ecologists will recognize this usage as being closer to the concept of "niche" than to the concepts of "habitat" or "biotope" (Hutchinson 1967; Whittaker, Levin $\&$ Root 1973). While humans and rats may share similar habitats, such as cities and farms, they occupy very different environments within those habitats. The natural" or "normal" environment of an animal will refer to that which it occupies by virtue of its phylogeny and species-typical ontogeny; statistically, one might define it as the modal (i.e. typical) environment of members of a species or population (Miller 1977). When the unqualified term "environment" is used in this paper, the qualification "natural" will always be implied.

The emphasis here (and in other ecologically oriented discussions of behavior) on animals' natural environments sometimes leads to misunderstandings. It is often thought, mistakenly, that any study that subjects an animal to conditions that deviate from its natural environment cannot qualify as "ecological." This belief misrepresents the role of the natural environment in ecological study, in which atypical or artificial conditions are often employed to advance our understanding of the relationship between an animal and its natural environment. Although the goal of ecological study is always to explain relationships with the natural environment, the experimental use of unnatural environments may be an important means of attaining that goal. This point will be elaborated later in this paper.

The distinction between general principles and general processes is also important to the arguments of this paper, and so I will briefly explain what is meant by it. Consider the following example, modified from Cabanac (1974). The thermal relationship between a homeothermic animal and its environment may be described by the following equation:

Qprod Qgain - Qloss $=$ O,

where $\mathbf{Q}_{\text {prod }}=$ metabolic heat production, Qgain $=$ radiative, convective, and conductive heat gain from the environment, and $Q_{1 \mathrm{os}} \mathrm{s}$, = radiative, convective, conductive, and evaporative heat loss to the environment.

Equation 1 expresses a general principle about thermoregulation that is true for homeothermic animals over some range of environmental temperatures characteristic of each species. Under different thermal regimes, various physiological and behavioral processes are initiated so that the principle in Equation 1 remains true, but in different animals, and in the same animal at different times, different processes will occur. For example, with an ambient temperature of $40^{\circ} \mathrm{C}$, the thermal balances of a man and a dog will both obey Equation 1, and both do so in part because of processes that increase evaporative heat loss. However, in man these processes include sweating (but not panting). whereas in the dog they include panting (but not sweating). By the same token, the man's thermal balance will also obey Equation 1 when the ambient temperature is $15^{\circ} \mathrm{C}$, but now the processes involved will include shivering, which increases metabolic heat production, rather than sweating.

The distinction between the principle expressed in Equation 1 and the processes by virtue of which the principle holds may be made as follows. Processes are events or series of events that are said to occur, to terminate, or to be observed. Those associated with learning may usually be described in either behavioral or physiological terms; physiological descriptions are conventionally said to underlie or reduce behavioral descriptions. Principles, on the other hand, are formal or informal statements about processes, relations among processes, or relations between processes and sets of external conditions. We speak of principles as being true, as being violated, or as being formulated The aim of theories is, in general, to explain or predict processes by means of principles.

The acceptance of a general principle such as that expressed in Equation 1 need imply nothing about the generality of the processes by virtue of which it holds In the preceding example there are some thermoregulatory processes that are very general (such as vasoconstriction and vasodilation) and others that are restricted to only a single species (such as putting on warm clothing). In a precisely analogous way, the search for general principles of learning does not commit one to the view that all learning reflects a general process (such as the formation of associations), and doubts raised as to the purported generality of certain processes of learning need not lead one to question the possibility of finding general principles.

The ecological approach to learning discussed in this paper shows how a search for general principles of learning may be undertaken quite independently of any assertions as to the existence of general processes. As will become clear in the final sections of the paper, the nature of the empirical research mandated by the ecological approach is radically different from that currently being undertaken by the majority of workers in animal learning.

\section{Contemporary approaches to the study of learning}

To attempt anything like a comprehensive survey of the current state of the psychology of learning would far exceed the scope of this paper, and the following account is intended only to highlight certain trends in the field that are particularly relevant to my arguments in the remainder of the paper. The reader interested in a more thorough treatment of the issues is referred to Rescorla \& Holland (1976) or Jenkins (1979) for a brief account, and to Bitterman, LoLordo, Overmier \& Rashotte (1979) for a more comprehensive survey.

One of the primary differences among modern workers in the field of learning is in the importance 
Johnston: Learning theory

that they attach to ecological and evolutionary considerations for the study of learning. For many years, the study of behavior was strongly influenced by a tacit belief in the complete separability (both conceptual and experimental) of learning and instinct - a belief that had, and continues to have, profound and farreaching effects in both biology and psychology (Kuo 1929; Lehrman 1970; Oppenheim, in press). On this view, instinct provides an animal with the capability of responding to situations that are typical of its natural ecology, whereas learning enables it to cope with atypical or unusual events. It is not surprising, given this conceptual position, that in the study of learning, little heed was paid to the subjects' natural ecology, and that subsequent development of the field left no room for ecological considerations to be taken into account.

I doubt that many modern students of learning would argue in favor of a learning-instinct dichotomy, but it can hardly be denied that the field as a whole continues to reflect the dichotomy, at least insofar as ecological considerations are denied any theoretical or methodological representation in the study of learning. Since about 1970, arguments have been presented against the ecologically arbitrary nature of work on learning, echoing the earlier concerns of such authors as Tinbergen (1951) and Lehrman (1962), and current opinion is clearly divided between those who argue that ecological differences among species are important to our understanding of their learning abilities and those who believe that such differences are of no great theoretical significance. Two current approaches to the study of learning that reflect these two points of view may be identified.

Miniature theories of learning. Most modern students of learning acknowledge that the concern of earlier theorists with large-scale theories, each of global applicability, was too ambitious. Attention has now turned to the construction of smaller-scale theories, each dealing with a restricted range of learning phenomena - a development that was anticipated by Tolman (1949). The work of Rescorla (1972; Rescorla \& Wagner 1972) on classical conditioning, of Sutherland \& Mackintosh (1971) on discrimination learning, of Kamin (1968; 1969) on expectancy theory, and of Bolles (1971; 1972) on avoidance learning, is representative of such miniature theories. With few exceptions, primarily Bolles $(1970 ; 1971)$, the proponents of this approach have paid little attention to ecological considerations in their analysis of learning. The methods that they employ for the study of learning are ecologically arbitrary in just the same way as were those employed by the general theorists of a generation ago, and theoretical analyses continue to offer no recognition of the different ecological circumstances with which each animal species must deal and to which it has become adapted as a result of natural selection. If anything, there is perhaps less interest now than previously in using laboratory investigation to determine how animals learn about the world in which they live (cf. Hull 1937). Indeed, the major difference between those working on the development of miniature theories and the general learning theorists of the past would seem to be procedural rather than conceptual, as Revusky (1977, p. 2) suggests:
"These students of learning typically believe' in the general process approach in principle, but feel that the only practical methodology, at least for the time being, is to construct theories limited to narrow situations and effects."

Although these theorists are certainly aware of species differences in learning ability, it is unclear what role, if any, they expect such differences to play in the completed theoretical formulation. One prominent modern learning theorist, M.E. Bitterman, has devoted much of his research effort to an analysis of differences in learning ability among different animal species (Bitterman 1975; 1976; Bitterman \& Woodard 1976). Of all the work being carried on in the tradition of general learning theory, Bitterman's comparative analysis is perhaps where one might most expect to find an interest in exploring the ecological correlates of learning, since few other investigators are so explicitly concerned with interspecies differences. That even his work reveals no such ecological content strongly suggests that the nonecological traditions of the field will not give way easily.

Biological boundaries of learning. Of more direct relevance to the present discussion is the second modern approach to the study of learning; the so-called "biological boundaries" approach. This approach draws its main support from various recalcitrant data, obtained in studies of conditioning, that appear to contradict several major assumptions of general process learning theory. The best known of these data is the finding of Garcia \& Koelling (1966) that rats will readily learn to avoid sweet-tasting water if its ingestion is paired with toxicosis, but not if ingestion is paired with foot-shock. By contrast, avoidance of water that is associated with an audio-visual stimulus can be learned by pairing its ingestion with shock but not with toxicosis. This result contradicts the principle of equivalence of associability of stimuli (Seligman 1970), according to which it is possible to pair any noxious US (unconditioned stimulus) with any CS (conditional stimulus) to produce aversion. Other findings (Garcia, Ervin \& Koelling 1966) showed that delaying toxicosis for up to two hours following ingestion still resulted in learning to avoid the sweet-tasting water. Again, this contradicts the principle that the US and the CS must be temporally contiguous if learning is to occur (Seligman 1970).

Since the publication of Garcia's original findings, and of papers by Seligman (1970) and Rozin \& Kalat (1971) emphasizing their theoretical significance, there has been a dramatic increase in the number of tasteaversion studies (see Riley \& Baril 1976). These studies, taken together, strongly support the view that the avoidance of noxious foods is mediated by different cues in different species. Many of the results suggest that the nature of the effective cues is determined by the way in which food is typically selected by members of a particular species. Thus quail (Wilcoxon, Dragoin \& Kral 1971) and vervet monkeys (Johnson, Beaton, \& Hall 1975), which may rely on visual cues in food selection, are able to learn to avoid noxious food on the basis of visual as well as gustatory cues. For the rat, a nocturnal scavenger, presumably visual cues are rarely 
available in the context of food selection, and so the ability to associate such cues with subsequent illness has never been selected for in the evolution of this species (Rozin \& Kalat 1971).

Although studies of taste-aversion learning have provided the bulk of the evidence in favor of the biological boundaries approach, substantial support has also come from studies of other forms of learning. Bolles (1970; 1971) has reviewed a wealth of evidence from the instrumental avoidance literature, which shows that rats will learn to make certain avoidance responses (such as running away or jumping out of the shock box) far more readily than others (such as turning a wheel or pressing a bar). Bolles proposes that each species possesses a limited number of species-specific defense reactions (SSDR's) that it uses under natural conditions to protect itself in any aversive situation, independently of any specific learning [see Bolles \& Fanselow: "A Perceptual-Defensive-Recuperative Model of Fear and Pain" BBS 3(2) 1980]. Only if the instrumental response required in an experiment permits the subject to use an SSDR to escape or avoid shock will learning occur. Complementary data, using food reinforcement in an operant conditioning paradigm with hamsters, are provided by Shettleworth (1975). Certain responses, such as digging, rearing, and scrabbling, can be reinforced by food, whereas others, such as face-washing, scent-marking, and grooming, may actually be depressed under food reinforcement. Shettleworth argues that the responses that can be reinforced are those that would normally occur in the context of feeding behavior in this species.

There are many other examples of such biological boundaries to learning ability, for which the reader may consult Breland \& Breland (1961), Seligman (1970), Seligman \& Hager (1972), Shettleworth (1972), and Hinde \& Stevenson-Hinde (1973).

Theoretical implications of the biological boundaries approach. At present there is no clear agreement on the implications of the biological boundaries criticism for the future development of learning theory. Some investigators (e.g. Bitterman 1975; Malone 1975) clearly feel that its impact on the general process view will be minimal. Others are more receptive but offer no very clear vision of the directions in which the psychology of learning should proceed. Thus Kimble (1973, p.12) acknowledges that "biological variables of many sorts have important messages for the psychology of learning," but he does not say what he perceives these messages to be, or how psychology should respond to them.

A few learning psychologists have responded more directly to the criticisms of the biological boundaries approach. Logue (1979) has reviewed the data from studies of taste-aversion learning and concludes that most require no radical reformulation of the accepted laws of learning, although some do require important quantitative modifications to those laws, especially in regard to the speed of acquisition and the long delay over which aversions may be formed. Logue concludes her review with a call for a more balanced approach to the study of learning: "This view recognizes the existence and utility of general laws of learning, but it also recognizes the necessity of acknowledging and investigating the dissimilarities in the learning of different species and the learning of different tasks. Otherwise we are likely to assume generality where none exists" (Logue 1979, pp. 290-91).

The heart of most current learning theory is associationism (Rescorla \& Holland 1976; Jenkins 1979), and it is therefore not surprising to find a serious attempt being made, by LoLordo (1979), to defend the principle of equivalence of associability against the claim that some associations are more easily formed than others. LoLordo presents a careful methodological critique of many of the experiments on which the biological boundaries criticism draws. He concludes that very few of those experiments demonstrate the selective formation of associations, and that the selective effects that they do reveal may be attributed to nonassociative phenomena such as cue salience, attention, and differential response elicitation.

LoLordo's analysis is especially interesting. If his defense of associative equivalence is correct, then it is possible to identify a wide range of selective nonassociative processes, of manifest importance to learning, that it is unconvincing to dismiss as mere speciesspecific "contaminations" (Schwartz 1974). If these processes have a major influence on an animal's ability to learn, as they apparently do, then our theories should reflect this fact and not view them as peripheral to "true" (i.e. associative) learning. It is, of course, quite legitimate for particular theories or investigators to focus their attention on associative rather than nonassociative phenomena, but if this is allowed to become the bias of the entire field of animal learning, then thee theories that result are likely to be of rather limited explanatory scope (Rescorla \& Holland 1976).

There is lack of agreement among the critics as well' as the defenders of traditional learning theory as to what the biological boundaries criticism implies for the study of learning. Seligman (1970), for example, proposed a dimension of preparedness (similar to the notion of belongingness proposed earlier by Garcia \& Koelling, 1966) for ordering associations according to the ease with which they may be formed. Somewhat arbitrarily, he delineates three types of association prepared, unprepared, and contraprepared - and

II

the formation of associations. The concept of prepared-' ness is a purely descriptive one, since there is nothing to tell us which associations are prepared until after we I have demonstrated a certain ease of formation (Schwartz 1,974). Nor, having demonstrated this, and identified certain associations as prepared, is it clear that very much has been gained over the general process account. The most that the concept of, preparedness seems to offer is an additional variable in the equations of learning (see Schwartz (1974) for further criticisms of preparedness). [See also Eysenck: "The Conditioning Model of Neurosis" BBS 2(2) 1979.]

Bolles's $(1970 ; 1971)$ concept of the species-specific defense reaction (SSDR) has already been discussed Although it appears at first sight to refer to a restricted type of prepared association (i.e. that certain avoidance responses are learned more easily than others), the 
SSDR concept is in fact somewhat more predictive than that of preparedness. We could, in principle, determine from naturalistic observation which responses an animal actually uses as SSDR's and then make predictions as to the ease with which various escape and avoidance responses could be acquired under experimental conditions. Although the applicability of the SSDR concept is limited to escape and avoidance conditioning, a similar concept could be developed for application to other forms of learning, such as that studied by Shettleworth (1975). In this case one would identify species-specific feeding behaviors, for example, and predict that these should be more easily reinforced by food than other, nonfeeding behaviors. However, the approach of testing predictions based on concepts like the SSDR has not yet been tried.

While Seligman and Bolles clearly hold out hope that some principles of learning, albeit of restricted generality, will be found to apply across species, other biological boundaries theorists seem much less optimistic. The dominant impression to be gained from the discussions of Hinde (1973) and Shettleworth (1972), for example, is that whatever principles of learning may be formulated, they are most likely to be limited in their applicability to one or a few species and situations. On this view, research inspired by the biological boundaries approach would consist largely in cataloging constraints on the learning abilities of each species of interest:

While it seems to be possible to delineate certain kinds of constraints on learning, it is probably not possible to predict where they will operate by translating directly from one case to another. However, as research in this area progresses, it may well become possible to characterize the constraints on learning and the cases in which they occur much more explicitly (Shettleworth 1972, p. 59).

Rozin \& Kalat (1971) occupy a position that is much closer to that which I shall develop in later sections of this paper. They stress the importance of considering learning as a component of an animal's adaptation to the specific requirements of its environment, but they are also quite optimistic about the emergence of at least some generality from these diverse data:

Given the constraints on adaptation produced by basic properties of the nervous system, the cost of evolving specializations, and the fact that most species face a common set of problems, we doubt that a separate learning mechanism would exist for every situation, or that there would be separate laws for each species (Rozin \& Kalat 1971, p. 481).

The view to be developed later in this paper is similar in spirit to that of Rozin and Kalat but, as will be seen, differs from it in some respects.

An important recent contribution to the biological boundaries debate has been made by Revusky (1977). Revusky is a strong proponent of general process theory who has met the challenge posed by taste-aversion studies head on. His own experimental work has focused on the long delay between stimulus (taste) and reinforcement (illness) over which taste aversions can be formed (Revusky \& Garcia 1970; Revusky 1971), and he has attempted to show that this phenomenon is compatible with a general process account of learning, in contrast to the claims of uniqueness by Seligman (1970) and Rozin \& Kalat (1971). Revusky (1977) is especially concerned, and rightly so, with the lack of any attempt by biological boundaries theorists to provide an alternative framework to general process theory within which we might search for general principles of learning:

The basic evidence used against general process learning theory seems to be lists of phenomena which do not fit into a known general process framework. Critics who use such "evidence," particularly Hinde (1973), do not seem disturbed by the fact that few of these phenomena can be explained by means of any other reasonably rigorous approach (Revusky 1977, p. 11).

Rozin and Kalat and other neoevolutionary [i.e. biological boundaries] learning theorists aggrandize minor science, the study of the particular, at the expense of extremely important science, the study of the general (Ibid., p. 10).

Like Logue (1979) and LoLordo (1979), Revusky is concerned to defend the assertions of general process theory by showing that many of the apparently contrary data may be accounted for in general process terms. An alternative response is to view the work of biological boundaries theorists not only in terms of the criticisms that it raises against general process theory, but also as suggesting an alternative approach to the study of learning that adopts a different point of departure from that of general process theory. Rather than starting from the position that the varieties of learning reflect an underlying general process, but one which may perhaps be modified in accordance with particular adaptive requirements, we might start with an explicitly ecological perspective on the problems of learning. In adopting such a perspective, we would be acknowledging the validity of the biological boundaries criticism, but using ecological considerations as a primary motivation in the study of learning rather than as a set of secondary, and largely peripheral, constraints. An approach of this kind would provide a framework that can motivate a search for general (ecological) principles of learning but that does not require allegiance to the associationist tradition, or to any other general process view. As these general principles become elucidated, we will be able to tackle the separate question of whether they reflect an underlying general process.

The question then arises whether the biological boundaries approach itself might not provide such an ecological alternative to the general process tradition. There are three main reasons for believing that it cannot, and these are discussed in the next section.

\section{Limitations of the biological boundaries alternative to general process learning theory}

Lack of generality. It may be the case, as many critics of general process theory imply, that there will be very few interesting generalizations to be made about learning, because of the wide diversity of phenomena that are revealed when learning is studied from a more 
Johnston: Learning theory

naturalistic standpoint. If that were to prove true, it would be extremely surprising, for there is a wealth of phenomena that have been studied from an ecological, naturalistic point of view and that have nonetheless yielded significant general insights. The literatures on population dynamics, predator-prey relationships, social organization, and evolution all show substantial theoretical development, based in large part on the study of particular, natural instances (see Mayr 1963; J. M. Emlen 1973; Alcock 1979). In some cases, general principles have been found to reflect general processes, and in others they have not. Given the primitive state of our knowledge about learning outside the general process tradition, it seems unduly defeatist at present to deny the existence of general principles. A far more productive strategy would be to admit that general principles may exist, although we cannot at present see what they are, and to consider how we might best go about searching for them.

In fairness to those whose work is being criticised, it should be admitted that part of the problem is to dislodge a weight of tradition that insists on the existence of general processes and to draw attention to adaptive specializations of learning that have been overlooked in the past. It is, however, much easier to dethrone a dominant paradigm, especially a long-lived one, if one has a viable alternative to offer (Kuhn 1962), and the biological boundaries theorists do not appear to have such an alternative.

Subservience to the general process tradition. Perhaps the greatest drawback to the biological boundaries approach is that it is less an alternative to general process theory than an addendum. As Revusky (1977, p. 12) points out, the very use of terms like "boundaries" and "constraints" implies that there is something (a general process?) that is being constrained. This is more than just a semantic observation: The two main theoretical proposals of biological boundaries theorists - Seligman's (1970) concept of preparedness, and Bolles's (1970) concept of the SSDR - look much more like supplements to the appropriate portions of general process theory than alternatives. A reasonable response by the general process theorist would be to continue the development of his general theory and to leave it to others to detail its application to particular species or ecological situations.

If the development of the poison-avoidance literature is a good indication of the kind of experimental work that is to support the biological boundaries approach, then we may also point to a methodological subservience to the general process tradition. Although some scientists may still believe in the conceptual neutrality of methodology (i.e. that "facts" exist to be "collected" by a convenient methodology, like collecting pebbles on a beach), this view has been almost universally abandoned, with good reason, by modern philosophers of science (see Kuhn 1962; Hanson 1969; Medawar 1969; Lakatos 1970). Methodology interprets the world, and it does so in terms of the theoretical predilections of its designers. Most of the experimental designs in the poison-avoidance literature are minor variants on themes developed by Pavlov, Thorndike, and their intellectual descendants. These designs were chosen for the study of learning precisely because they are biologically arbitrary (e.g. Thorndike 1911 , p. 30 and to prevent the subjects from relying on "the helping hand of instinct" (ibid) in solving the problems posed. This is not to say that modern psychologists necessarily subscribe to a separation of learning and instinct; but in searching for an alternative to (rather than merely a criticism of general process theory, it seems counterproductive to employ the methods that have been sanctioned by that theory. There is a very real danger that these methods force an interpretation of the world in the very theoretical terms to which we seek an alternative.

An illustration of the power of the general process tradition is provided by the introduction to a recent poison-avoidance study by Galef $\&$ Osborne (1978). They sought to investigate the hypothesis that aposomatism (bright coloration) in many poisonous insects is an adaptation to the ability of potential predators to learn to avoid such prey, which implies that predators should indeed demonstrate the appropriate learning ability. Clearly that is just the kind of hypothesis whose investigation might promote a biological alternative to general process theory. Having presented their hypothesis, the authors discuss their choice of an experimental species:

The choice of a species in which to test the preceding hypothesis poses something of a problem. It might well be argued on ecological grounds that, given the rationale for the present studies, Rattus norvegicus would be a particularly inappropriate choice. Not only have we failed to find evidence in the literature that rats encounter aposomatic prey in the wild, but in addition it is well established that rats feed most frequently in hours of darkness when visual cues are difficult to utilise. On the other hand, models of poison avoidance learning in the psychological literature most frequently treat the rat as representing the general mammalian case, from which, for example, one extrapolates to poison avoidance of humans or that of coyotes. The taste aversion learning of other species is by contrast viewed as, to some extent, idiosyncratic and specialized.

Thus the species chosen for the present work is necessarily undesirable from either the psychological or the ecological point of view. Because the work described below was more directly addressed to questions concerning the proximal causation of behavior than its function, we made the ecologically inappropriate choice of subject species (Galef \& Osborne 1978, p. 908; emphases added).

Given the rationale for the study, the reference to "proximal causation" in the last sentence can only imply a belief in some general process underlying poison avoidance, which is equally well revealed by studying any form of poison avoidance in any species. Although this may be a somewhat extreme example, it illustrates the power that general process theory may have to constrain experimentation in the absence of any alternative theoretical framework. 
Ex post facto adaptive explanations. It is extremely difficult to test hypotheses about the presumed adaptiveness of a biological trait, especially when it is not amenable to direct manipulation, as in the case of a learning ability. Unfortunately, it is very easy to think up plausible adaptive stories about almost any characteristic of an organism, and such stories tend to prevent any real ecological interpretation of the characteristic in question. This is widely recognized as a persistent deficiency in much evolutionary writing (Ghiselin 1974); in some areas, such as the rapidly developing field of sociobiology, it has reached epidemic proportions (Lewontin 1979). [See also Ghiselin: "Categories, Life, and Thinking" BBS 4(2) 1981 (forthcoming).]

A primary aim of the biological boundaries approach to learning is to offer an explanation of learning in terms of its contribution to an animal's adaptation to its environment - an aim that it shares with the ecological approach that is developed later in this paper. However, if such explanations are to be truly revealing, they must be based on analysis, not solely on conjecture (although conjecture may of course be a source of fruitful hypotheses), and such analysis has been lacking in the biological boundaries approach. Thus, while it may be reasonable to argue that primates can learn to associate visual cues with nausea because they normally select food on the basis of such cues (Johnson et al. 1975), in the absence of an empirical demonstration of how primates do in fact select food, this remains a hypothesis, not an explanation. Similarly, the fact that hamsters can be reinforced with food for digging, scrabbling, and rearing may be due to the occurrence of such actions during feeding (Shettleworth 1975), but this needs to be demonstrated by observations of naturally occurring behavior.

It may be objected that the adaptiveness of such learning phenomena is either self-evident or readily apparent from casual observation, and that there is no need for detailed and time-consuming ecological analysis. Claims of self-evidence in science are of course best treated as hypotheses, since they often turn out to be false. Inferences about adaptiveness based on casual observation should likewise be treated with extreme caution, since it has proved difficult even to define criteria for identifying adaptive behavior (Hinde 1975; Lewontin 1978; Gould \& Lewontin 1979; GluttonBrock \& Harvey 1979). The adaptiveness of Batesian mimicry, in which a noxious model is mimicked by a palatable species, might be considered "readily apparent," and yet, despite detailed ecological study (e.g. Brower, Cook, \& Croze 1967), it is only recently that convincing evidence of that adaptiveness has been forthcoming (Jeffords, Sternburg \& Waldbauer 1979). It is true that an experienced investigator can often make accurate inferences about the adaptive value of an animal,s behavior, but the ease with which incorrect, though plausible, adaptive stories may be devised (Lewontin 1979) suggests strongly that such inferences should be framed as hypotheses rather than as explanations. As more complex forms of learning attract the attention of biologically motivated investigators, the 1 fingers inherent in ex post facto adaptive explanations 1 will increase.

\section{A sketch of an ecological approach to the study of learning}

If it is agreed that science should aim for generality of explanation, then, other things being equal, a single theory of learning is to be preferred over a variety of models of limited applicability. My aim in the remainder of this paper is not to attempt to formulate a general theory of learning, but rather to indicate how such a theory might be sought. My argument is that, to do this, we must follow the lead of those who claim that learning is to be understood in terms of adaptation. At the same time, we must avoid arguing, as some have done, that particularity of process implies particularity of principle, as urged in the introduction to this paper. If we can do this, then we will be able to discover whatever general processes of learning may exist, but we will not overlook important adaptive specializations of learning, which is the major criticism voiced by biological theorists against general process learning theory.

The question of central importance in the ecological approach to learning is: What do animals learn and how do they learn it? The first part of this question (What do animals learn?) reflects a concern with the problems faced by animals in their natural environments; the second part (How do they learn it?), with the ways in which experience contributes to the solutions of those problems. The general process approach has concerned itself almost exclusively with the second part of the question. Anything that an animal can learn, regardless of whether or not it actually does learn such a thing under natural conditions, might be selected for study by a general process theorist; his choice would be constrained by convenience (Skinner 1950) and tradition, not by any consideration of whether the task is a natural one for the animal under study. A similar emphasis is evident in other fields concerned with the development of behavior. Gottlieb has remarked on

. . . the banality of recurrent demonstrations of experiential modification or experimental modifiability, when these demonstrations are attended by a failure to relate the significance of such modifications to the normal (usual) route or outcome of species-typical development. . . In the literature on this topic, there seems to be the tacit assumption that, if a given feature of neural or behavioral development can be shown to be susceptible to the influences of experience, then experience must normally play a part in its ontogeny. (Gottlieb 1976, pp. 48-49)

McCall (1977) expresses a similar point of view in regard to developmental psychology.

Gottlieb's caution applies equally to the study of learning: Simply showing that an animal can learn a particular task does not allow us to say that it actually does learn the task, or even any similar task, under natural circumstances. In order to be able to make this assertion, we must study the animal under natural conditions, or under some close approximation to them in the laboratory (Schneirla 1950; Miller 1977; Petrinovich 1979). 
Some readers will no doubt object that psychologists are primarily interested in studying the mechanisms of learning, rather than the ways in which those mechanisms are employed under particular natural circumstances. It is frequently unclear what is to be understood by the term "mechanism" in this context. Not all persons- who declare such an interest are concerned with the physiological bases of learning (one possible definition of "mechanism"), and the term requires some clarification before the strength of the objection can be properly evaluated. However, whatever interpretation is given, an important problem remains. The objection implicitly assumes that there is some underlying process (or processes) of learning that does not change as a function of what the animal is learning to do (that is, it assumes a fairly strong version of the general process view). Such an assumption is absolutely required of an argument which claims that "the mechanisms" of learning are equally well revealed by any task situation, regardless of how closely it approximates one that is a natural task for the animal. This assumption is by no means logically necessary; in fact, it is a very strong empirical claim that surely remains to be demonstrated. While it may be true, it cannot be assumed to be true. In searching for a general theory of learning, we require an approach that can determine the truth or falsity of that claim, rather than assuming its truth.

If the assumption of a common mechanism underlying learning is mistaken, then a serious problem arises with any approach that assumes it - namely, the possibility of creating behavioral artifacts. The study of behavior is a part of natural science and, as such, is concerned with the explanation of natural phenomena. Distinguishing natural from artifactual phenomena is frequently very difficult in practice, but in principle it is quite straightforward: Natural phenomena are those that exist independently of our investigations of them. When we make investigations, it is therefore important to determine which of our observations reflect the natural organization of the phenomena we are studying, and which are artifacts that reflect only the peculiarities of our investigative methods. When an anatomist studies the structure of a particular cell type, for instance, he may use various staining and fixing procedures to highlight certain aspects of structure and suppress others. It is well known to anatomists that the incautious use of many of these procedures produces artifacts that are highly reproducible and that can easily be mistaken for natural phenomena. The study of behavior, of course, carries with it no guarantee of exemption from this danger. The fact that an animal behaves in a regular and reproducible manner in an artificial laboratory environment does not mean that what we observe in such an environment necessarily demonstrates anything about the natural organization of behavior. The regularities observed may be imposed on the animal's behavior by the characteristics of the situation and may have nothing whatever to do with the regularities of natural organization that we seek to describe and explain.

This danger has been pointed out by Seligman (1970) and Petrinovich (1979), and the ecological approach strives to avoid it by ensuring that the phe- nomena of learning that we study are natural onesthat they do in fact exist independently of our investigations of learning. Such an approach therefore begins by analyzing each example of learning as an adaptation to a natural environment, rather than introducing adaptive considerations after the fact of laboratory studies (see the preceding remarks on ex post facto adaptive explanation).

The conception of adaptation. The concept of adaptation is central to any ecological study; it is a formal statement of the intuition that biologists have had since well before Darwin that animals are in general very well equipped to cope with the demands of their environments. In pre-evolutionary biology, that fact was seen as the result of providential design; since Darwin, it has been interpreted as the outcome of natural selection. Despite its importance in ecological study, and in biology in general, finding a broadly acceptable definition of adaptation has proven very difficult (see Bock \& von Wahlert 1965; Dobzhansky 1968; Brandon, 1978; Lewontin 1978; Bock 1980). The most complete analysis of adaptation is that provided by Sommerhoff (1950; see Johnston \& Turvey 1980).

In Sommerhoff's account, an animal's adaptation to its environment is expressed in terms of its ability to attain a set of goals in that environment. The nature of those goals depends, of course, on the animal under study; they might include orientation to a nest site, capture of a certain kind of prey, courtship of a mate, or defense against certain predators. In general, they comprise whatever states of affairs must be brought about, typically recurrently, in order that the animal may survive and reproduce. The animal's ability to attain those goals depends upon various physiological, anatomical, behavioral, and/or developmental characteristics that together constitute the biological support for adaptation, and the analysis of particular cases of adaptation involves the specification of the pertinent characteristics. The technical and conceptual difficulties involved in such endeavors are considerable and have been discussed by Hinde (1975), Lewontin (1978; 1979), Gould \& Lewontin (1,979), and others.

Two points that have been stressed by recent authors' are particularly relevant to the present discussion. The first of these is that not all characteristics of an organism necessarily contribute to the biological support for adaptation, and those that do may not be optimally designed for the adaptive role that they play. Characteristics may be vestigial, such as the vermiform appendix in man, or they may show adaptively neutral variation, such as eye or hair color. Even in the case d adaptive characteristics, there may be internal constraints on development or on the organization of the genome that preclude selection of an optimal; design in the course of evolution (Lewontin 1978). The ecological study of learning must therefore take very seriously the possibility that not all forms of behavior or 1 behavioral change can be considered to be adaptive. The fact that an animal can learn to perform a task is no guarantee of an adaptive role for either the task or the learning ability.

The second point is that adaptation is an inherently relational concept: When we speak of some character- 
istic of an organism as being adaptive, we necessarily imply an environment to which it is adapted. If we are to reach an understanding of learning as, or in terms of, adaptation,' then we must pay close attention to the nature of the environments to which particular kinds of learning are adaptations (Slobodkin 1968; Slobodkin \& Rapoport 1974).

The nature of the environment: task description. Like all other approaches to the study of learning, the ecological approach seeks an answer, or set of answers, to the question: "How do animals learn?" But from the ecological point of view, we see that the question cannot be answered in that form. Different animals have different adaptive relationships with their environments, and so the question must be broken down into two questions, to be answered separately for each animal species. The first of these takes the form: "What are the behavioral problems that this animal must solve in adapting to its environment?" The second question takes a more familiar form: "How does the animal learn to solve those problems?"' Not only are questions of the first kind equal in importance to those of the second, they also clearly have logical priority. To draw an analogy, if we were to ask how to design a bridge, an engineer's first question, of necessity, would be: "What is it that you wish to bridge?" A question of this kind is a request for a task description, and task descriptions play an important and fundamental role in the ecological approach to the study of learning.

The term "task description" comes from the literature of artificial intelligence (e.g. Simon 1969). In order to write a program to solve a particular problem, one requires a detailed analysis of what the problem is. In many cases (for example: Compute $2+2$ ) the task description is quite straightforward. In other cases (for example: Play a good game of chess) writing the task description may be a major part of the whole endeavour. The problem of writing task descriptions for learning is far more likely to resemble the latter of these two examples than the former (see further below).

The claim that descriptions of "What?" are logically prior to and as important as analyses of "How?" has been made by Gibson (1966) in his ecological theory of perception, and before him by Brunswik (1952). Gibson points out that, outside the psychological laboratory, animals (including humans) do not perceive brief, isolated flashes of light; rather, they perceive objects and events that are revealed by patterns of stimulation in a dynamically changing, richly textured optic array. He argues that it is surely an empirical question whether the means by which we perceive the former stimuli are the same as those by which we perceive the latter, and that in order to answer this question we need to know what is being perceived in the natural case. That is, we need a task description for the perceiving organism. [See also Ullman: "Against Direct Perception" BBS 3(3) 1980.]

My argument in regard to learning is similar to Gibson,s in regard to perception. In order to answer the question "How does this animal learn?", we must first ask "What does it learn to do?" (see also Petrinovich 1979).
Task descriptions in the study of learning. It must not be supposed that the formulation of task descriptions for the study of learning will be an unproblematic enterprise. On the contrary, determining in useful detail what it is that animals learn is likely to require as much effort and ingenuity as that already devoted to the question of how they learn. Several authors have seriously addressed the issue of task descriptions (although not by that name) in their studies of learning, and a brief consideration of some of them will illustrate what may be involved.

Charlesworth $(1976 ; 1978 ; 1979)$ has studied the nature of the problems typically encountered by young (2- to 4-year-old) children in their home environments. As part of a larger research program designed to analyze the "survival or adaptive value of intelligence" (Charlesworth 1979, p. 212), the description of those problems corresponds to the task-description stage of an ecological study of learning. It answers the question: "What are the problems that young children typically solve?" Further research will clearly be needed to determine how children learn to solve those problems, but Charlesworth's research already suggests that serious concern with task descriptions may lead to a picture of the child's intelligence rather different from that provided by the traditional approaches. For example, he notes that "very few instances of complex problems, such as those used in psychological testing or tool-using studies, were observed" (Charlesworth 1979, p. 214; emphases added).

The work of S. T. Emlen on the development of migratory orientation in the indigo bunting (Passerina cyanea) is perhaps a paradigmatic example of the ecological approach to the study of learning (reviewed by Emlen 1972; 1975). The first studies in Emlen's research program (Emlen 1967a; 1967b) involved the task-description stage; they answered the question "What behavioral problems are involved in migratory orientation for the indigo bunting?" The bunting is a nocturnal migrant; by placing mature, ecologically experienced birds under a planetarium dome during the normal migratory season, Emlen was able to arrive at a precise description of the orientation skill. Briefly, configurational information distributed over groups of stars, especially those within about $10^{\circ}$ of the pole star, provides the basis on which the bird selects an initial migratory direction (Emlen 1967a; 1967b). Arriving at the task description required information on the species' habits and life history to determine what orientation cues might be available to it, together with experimental manipulation to determine which of those cues are actually used for orientation. On the basis of this ecological task description, Emlen was able to proceed with an investigation of the second question: "How is this orientation skill learned?" (see below).

It is interesting to contrast the ecological approach in the study of learned orientation in a particular species with that adopted by traditional learning theorists in the study of learned orientation as a general, supraspecific problem. When Small (1901) used mazes in his studies of rat learning, he did so on the plausible, though so far as I know undemonstrated, assumption that a maze is a close approximation to the burrow systems in which rats live - that is, he attempted to set up a task 
Johnston: Learning theory

description for this kind of learning. Subsequent development of Small's work eliminated most of the ecological content of the task description and reduced the problem to one of "behavior at a choice point" (Tolman 1938). The relation of the logical conclusion of this development (the $\mathrm{T}$ maze) to any problem actually faced by the rat is obscure and has certainly received no attention by general process theorists. The methodological trend in this area of learning research has been to emphasize the logical structure of the task to the complete exclusion of its ecological structure. That animals normally learn about logic is, I think, debatable, although if they learn anything at all, they must learn about their ecology. Since different species have different ecologies, they may well learn different things, even within the general domain of orientation. The orientation problems faced by a rat and by a migrating bunting are very different, and it is not clear that anything is gained by attempting to strip them of their ecological content and reduce them to problems of logic. My general point is supported by Lynch's (1961) classic study of human orientation in cities, which demonstrated that the ecological structure of a city may be much more important than its geometric structure in determining how people find their way around. The geometric structure of a city is ecologically neutral in the same way the logical structure of a learning task is. [Cf. Olton: "Hippocampus, Space and Memory" BBS 1(3) 1979, and O'Keefe \& Nadel: The hippocampus as a cognitive map, BBS 2(4) 1979.]

What general guidelines do these examples offer for the construction of task descriptions? It is of central importance that task descriptions start with the study of the animal,s behavior under natural, species-typical conditions, but it is unlikely that naturalistic observation can provide all the required information. In many cases, experimental study will be necessary, especially to determine the sources of information in the environment with respect to which the behavior of interest is controlled. Some readers may feel that the requirement of naturalistic observation limits the ecological study of learning to individuals who have the necessary training and interest in the field study of behavior; but, in fact, many of the data from which task descriptions could be constructed are already available in the ethological literature. In particular, the problems of orientation and feeding have long been the concern of behavioral ecologists, and numerous excellent studies of the ways in which these problems are solved in many different species have been published (see J.M. Emlen 1973; Brown 1975; Alcock 1979). The ecological study of learning need not be forestalled by any lack of data on which to base task descriptions.

Local principles of adaptation by learning. The provision of a task description for behavior is only the first stage, albeit a most important one, in an ecological approach to the study of learning. Having answered the question "What does this animal learn to do?", we can approach the question that is of central importance to any student of learning: "How does it learn to do that?"

Generally speaking, the answer to this question involves a specification of the contributions of experi- ence to the development of behavior (as given by the task description) and an analysis of the means by which such experience has its effects (ultimately, though not necessarily immediately, in physiological terms). More specifically, we might seek information on any of the following points (this is not an exhaustive list):

1. What are the necessary and sufficient experiential conditions for the normal development of this behavior?

2 . Is the experience required, or most effective, at ${ }^{11}$ certain times if learning is to occur?

3. How do different experiences interact in the development of the behavior?

4. How does the development of one behavior interact with that of another?

5. In what ways does early learning constrain learning that is possible in later life? In particular, is the acquisition of certain behavioral skills necessary before others can develop?

6. Are the effects of experience primarily maintaining, facilitative, or inductive?' (Gottlieb 1976).

Because the behavior whose development is being analyzed has been given an ecological task description (e.g. feeding in a certain manner on certain foods), the kind of analysis implied by the above questions is implicitly undertaken in an adaptive framework. The fact that these questions reflect an ecological/adaptive approach to the analysis of learning must not, of course, be taken to mean that their answers demonstrate the adaptiveness of either the behavior or the means whereby it is learned. Determining the adaptiveness of learning is an extremely difficult theoretical and experimental problem (Tinbergen 1963; Hinde 1975; Lewontin 1978) that will not be considered here. A partial treatment of this problem will be published elsewhere (Johnston, in press).

The kind of analysis implied by the six questions listed above, and others like them, deliberately blurs the distinction between development and learning (Johnston, in preparation). The concept of development as a passive unfolding or maturation under genetic control has been almost universally rejected on the basis of repeated demonstrations that nearly all development shows some important dependence on experiential factors (see Kuo 1922; Lehrman 1953; Schneirla 1956; Gottlieb 1976; Oppenheim, in press). In more recent usage, "development" has come to imply the ontogeny of species-typical behavior, whereas "learning" often refers to the acquisition of the highly artificial kinds of behavior studied within the general process tradition. (This is, of course, a question of emphasis rather than of definition.) If the ecological approach to learning is to be successful, it is clear that this distinction can no longer be maintained. Many of the phenomena traditionally analyzed as instances of "development" will undoubtedly turn out to have important implications for an ecological theory of learning and should not be excluded from the scope of such a theory by arbitrary definition (Johnston \& Turvey, in press).

A few words should be said here concerning the methodological approach required for an ecological analysis of learning. There is nothing in the ecological attitude that precludes the use of artificial situations for 
the study of learning. Indeed, without manipulating an animal,s environment, it is clearly impossible to determine the role of various kinds of experience in the development of its behavior. The ecological approach does demand, however, that such artificial situations be used to test hypotheses about learning as it occurs naturally, rather than serving as objects of study in themselves. The insights to be gained from the use of artificial conditions depend on the contrast with development under normal circumstances, and the definition of normal circumstances must obviously be based on ecological description (Gottlieb 1976, pp. 48-9; Miller 1977).

The aim of the analyses I have been discussing is to provide a set of local principles of adaptation for each species, behavior. The ecological task description for each behavior of interest specifies the nature of the adaptation to be accounted for; it describes the way in which the animal solves a particular adaptive problem, such as feeding, orienting, or communicating with conspecifics. The aim of the analysis is to specify how this solution is reached in the course of development. The results of the analysis might be expressed as a model (not necessarily formal), which is an explicit statement of the principles by which the animal achieves its ontogenetic adaptation to some aspect of its environment. These principles are termed "local" because there is no way to determine, without further analyses of other species, whether they apply to more than the single species in which they are described. The problems of arriving at more general principles of learning are discussed in the following section.

A good example of the way in which the search for local principles of adaptation may be carried out is provided by Gottlieb's (1971) studies of the development of auditory species identification in ducklings. Space precludes a comprehensive review of these very extensive experiments; what follows is a selective account. Incubator-hatched, maternally naive Peking or mallard ducklings (Anas platyrhynchos) show a strong tendency to approach the mallard maternal call 24 hours after hatching, preferring that call to the maternal calls of other species (Gottlieb 1971). Selective approach to the conspecific call is clearly a skill of considerable adaptive importance to young ducklings, who rely on their mother for warmth and protection for some time after hatching (Collias \& Collias 1956; Miller \& Gottlieb 1978). Gottlieb's studies concern the role of prenatal auditory experience in the development of this species-typical auditory preference. Normal Peking ducklings start to vocalize in the egg at about 24 days of incubation, three days before hatching. Embryos that are surgically devocalized before that time fail to show the normal postnatal auditory preference for certain features of the maternal call, in particular for the species-typical repetition rate of 4 notes/second (Gottlieb 1978). Since the embryos show a selective response (diminution of bill-clapping; Gottlieb 1979) to the mallard call before the onset of self-produced vocalizations (at about 22 days of incubation), the role of those vocalizations is to maintain a preexisting auditory preference (Gottlieb 1976). If a tape recording of the embryonic call is played to devocalized embryos, the postnatal preference for the maternal call is reinstated (Gottlieb 1980), confirming the role of self-produced stimulation in the development of this adaptive behavioral skill.

Gottlieb,s results do not reveal the kinds of processes that would be called "learning" by someone working within the general process tradition, but they are the kind of finding with which an ecological approach to learning must be prepared to deal, since they demonstrate an important role for experience in the development of an adaptive behavioral skill.

A second example of the search for local principles of adaptation comes from the work of Emlen (1972) on the development of migratory orientation in the indigo bunting (discussed earlier). Emlen (1969) found that when young birds were prevented from viewing the night sky in their first year (prior to the onset of any migratory tendency), no directional preference was exhibited in the following migratory season - an example of an inductive effect of experience (Gottlieb 1976). Further study (Emlen 1970) showed that exposure to a rotating star field is crucial to the development of normal directional preference; exposure to a stationary star field does not support normal development. A point of further interest is that it does not matter whether the star field rotates about the normal pole star (Polaris) or another star (Betelgeuse); birds exposed to a star field rotating about Betelgeuse subsequently take their bearings from that star rather than from Polaris.

These two examples illustrate two important points that were made in the previous discussion. The first is that ecological analyses of learning are based on ecological task descriptions. The role of the task description in Emlen's work has already been described. In Gottlieb's research its importance is shown by the fact that Peking ducklings will only develop a preference for the species-typical call; exposure to altered calls will not induce a nontypical preference (Gottlieb 1980). Without a task description (i.e. an answer to the question: "What preference normally develops?"), it is clear that the development of auditory preferences in this species would hardly have been susceptible to an experimental analysis.

The second point concerns the use of artificially modified environments in ecologically motivated analyses of development: Both series of studies utilized sophisticated experimental techniques, including surgical manipulations, to elucidate the role of experience in these instances of development. I emphasize the latter point because it is often believed, mistakenly, that the ecological study of behavior is necessarily limited to naturalistic observation and precludes experimental analysis in the laboratory. What is precluded by the ecological approach is the use of ecologically arbitrary experimental designs for such analyses.

The results discussed here come from two illuminating lines of research out of many that might have been selected. Other examples that illustrate the ecological approach to the study of learning would include the studies of bird-song learning by Marler and his colleagues (Marler 1970; Nottebohm 1970; Marler \& Mundinger 1971; see Marler 1977, for some general theoretical principles that may be drawn from this work) and the many studies of feeding behavior that have taken ecological data as a starting point (e.g., 
Hogan 1977; Pietrewicz \& Kamil 1977; 1979; Kamil 1978; Gray \& Tardif 1979). [See also Toates: "Homeostasis and Drinking" BBS 2(1) 1979.]

Global principles of adaptation. The discovery of local principles of adaptation is clearly an enterprise of considerable scientific importance in its own right. The search for a general theory of learning, however, demands that we also attempt to discover principles of ontogenetic adaptation that apply to more than a single species or situation. In the past, such global principles have been presumed to be best revealed by isolating the animal from the specific demands of its natural environment. Implicit in this strategy is the assumption that principles of learning apply to the animal alone, hence that ecological considerations are peripheral to an account of learning. By contrast, the ecological approach views learning in terms of adaptation, so that its understanding rests on an analysis of both the animal and its environment. This strategy has been explicit in the preceding discussions of task descriptions and of local principles of adaptation. The search for global principles demands an equally explicit ecological strategy.

The probable importance of an ecological strategy in accounting for learning as a natural phenomenon may be seen by considering the general finding that emerges clearly from many studies of learning that have been conducted by ethologists: In many cases where experience has been shown to play a role in behavioral development, only normally occurring, species-typical experience (that provided by the natural environment) permits the development of adaptive behavior. Indigo buntings must be exposed to orientation cues early in development, but specific natural cues (a rotating starfield) are required for normal development of the orientation skill (Emlen 1970). White-crowned sparrows will not develop song without exposure to a song model, but that model must correspond to the species-typical song; other songs will not be learned (Marler 1970). Red-legged frog tadpoles require early exposure to patterned substrates if they are to acquire a substrate preference, but preferences for a striped (natural) substrate pattern are acquired more readily than preferences for a checkerboard (unnatural) pattern (Wiens 1970).

It is very hard to see how a nonecological theory of learning could ever account for all of the above examples without postulating various ad hoc exclusionary rules to fit the manifest ecological constraints on each species' learning ability. Whether an ecological theory can give a satisfactory account of these examples remains to be seen, but ecological principles of some kind are clearly needed to reflect the special role that the natural environment plays in each case. Many examples of learning, it appears, are closely tailored to the nature of the specific environments in which the learners live. In the terminology of Bekoff $\&$ Fox (1972), development is both environment-dependent and environment-expectant, since it expects the particular environment on which it depends. Understanding learning, therefore, requires description and analysis of the environment that is learned about; it requires an ecological approach to the study of learning as adaptation.

By considering learning as an instance of adaptation, we imply a viewpoint from which learning is seen as a process that ensures a correspondence between an animal's behavioral skills and the requirements of its natural environment. From the point of view of adaptation, the animal and its environment stand to one another in a complementary relationship, and following the notation of Turvey \& Shaw (1979), we may write this relationship as:

\section{Animal o Environment.}

The terms "animal" and "environment" in such a relationship are variables that range over a number of species and ecological niches, respectively. To the extent that animals are in fact adapted to their environments, the complementarity relationship o holds for natural ecosystems (i.e. animals and their natural environments). It may also hold for some artificial ecosystems, since an animal may well be adapted to environments other than its natural one, but here I shall consider only the natural case.

Typically, we would not attempt to specify all of the aspects of organismic and environmental structure in such a relationship. Rather, we identify some aspect of the animal (such as its learning ability) as being of special interest and describe that aspect in relation to appropriate aspects of the environment. In the ecological study of learning, basing analyses of learning on ecological task descriptions helps to ensure that the appropriate aspect of the environment has in fact been described.

The problem that we face is that of replacing the symbol o by a principle of learning that remains invariant over all (or at least many) species; from the preceding discussion it can be seen that this might best be achieved by allowing "animal" and "environment" to covary within the constraints imposed by the structure of natural ecosystems. On this view, general principles emerge by virtue of the complementary diversity among animals and their environments, not in spite of the diversity among animals considered alone.

The increased generality that is provided by an ecological approach to the analysis of animal structure and function is well illustrated by Gordon Walls, s classic volume, The Vertebrate Eye and its Adaptive Radiation (1942). Roughly half of Walls,s book is devoted to an ecological analysis of optic structure, in which the problems of vision in different environments (diurnal, nocturnal, aquatic, terrestrial, etc.) are discussed with reference to the eyes of animals that inhabit them. A number of interesting general principles emerge from this undertaking - principles that clearly could not have emerged if the various species had been considered in isolation from the environments to which they have become adapted. Walls does not provide formal expressions of these principles, but an informal example will suffice to make the point.

The shape of the lens shows considerable variation among different species of vertebrates. In some, such as man, it is greatly flattened, with a flatness index (diameter divided by thickness) of about 2.7. In others, 
such as most fishes, it is more nearly spherical, with an index of about 1.0. Broadly speaking, the lenses of terrestrial animals are much flatter than are those of aquatic ones, and an explanation of this diversity may be given in ecological terms. In air, light is strongly refracted at the cornea and needs only weak refraction at the lens to reach a focus on the retina - hence a flattened, weakly refracting lens in terrestrial vertebrates. However, in water, light is hardly refracted at all at the cornea and, in consequence, must be refracted more strongly at the lens - hence a spherical, strongly refracting lens in aquatic vertebrates. The general principle implied here holds true in the case of related species with contrasting habits: The spiny anteater (Tachyglossus), a terrestrial marsupial, has a flatness index of 2.75, whereas the duck-billed platypus (Ornithorhynchos), which is largely aquatic, has an index of 1.4 (Walls 1942, p. 669). A particularly interesting test case is the South American four-eyed fish (Anableps). This animal has a remarkable ocular anatomy related to its habit of swimming on the water surface with the top of its head protruding into the air. Each eye has two pupils, one looking up into the air and one down into the water. Light passing through each pupil is focused onto a separate retina by a single lens occupying the middle of the eyeball. The lens is oblate, having a high flatness index when measured in regard to the upward-looking (terrestrial) pupil and a low index in regard to the downward-looking (aquatic) pupil. The peculiar anatomy of the Anableps eye is readily understood in terms of its natural habitat, which lies at the interface of two environments with very different optical requirements (see Walls 1942, frontispiece and p. 434).

The general principle that explains variation in lens shape in terms of the refractive properties of different ecological media is unlikely to provide a full accounting of such variation, but it does illustrate the point that a substantial amount of generality can be extracted from structural diversity by an appeal to ecological considerations. In the ecological study of learning, a search for general principles requires a similar strategy. Rather than being a search for invariant properties of animals (as in the general process approach to learning) it is a search for invariant relationships between animals and their environments. Adopting such an approach, we might find, for example, that the number of encounters (trials) that an animal requires in order to learn to avoid noxious food items is a function (possibly complex) of the harmfulness of noxious items, the relative frequency of noxious items, and the energetic costs associated with the capture of a single food item. The independent variables in such a general principle are, of course, specified as part of the task description, not on the basis of arbitrary laboratory manipulations.

Note that any such general principle is quite neutral with respect to the existence of general processes of learning, as was also the case with the thermoregulatory example discussed earlier. It might be that a species whose environment includes very harmful potential food items learns the identity of correct food by "imprinting" food brought to it by its parents (and hence presumably safe), whereas a species for whom even the most noxious food item is relatively harmless may learn more slowly, by means of "association." Here "imprinting" and "association" are labels identifying local principles of adaptation that reflect different underlying processes of learning. The general principle is not made any less general by virtue of this fact, and, of course, some (or even many) ecological general principles may indeed reflect common processes among the local principles on which they are based. In the ecological approach, the existence of such general processes becomes a matter for demonstration rather than assertion.

The relationships among the three levels of ecological inquiry into learning, task description, local principles of adaptation, and global principles of adaptation, are illustrated in Figure 1. This figure emphasizes that, in contrast to the biological boundaries approach, the ecological approach is inherently ecological; ecological considerations are not simply brought in after the fact to explain data that do not accord with the general process view.

An important point concerns the question of the dimensions along which we should search for global principles among a diversity of local principles of adaptation. It would be premature to attempt to offer a definitive answer to this question, but I shall briefly

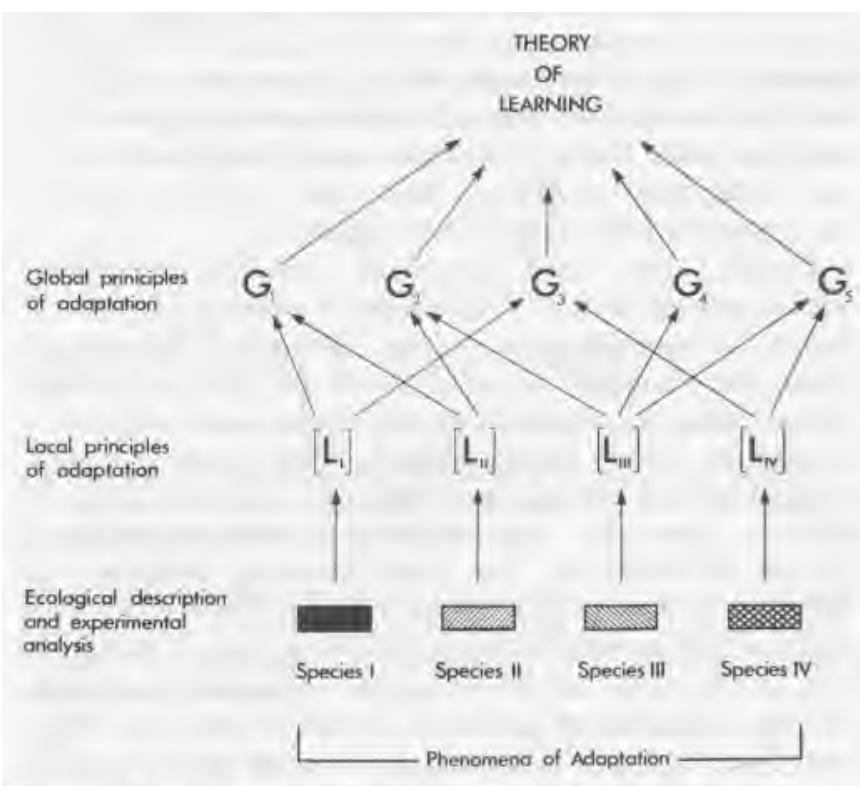

Figure 1. The ecological approach to the study of learning. The four shaded rectangles at the bottom of the figure represent four natural ecosysterns (animals and their natural environments). Description and analysis of the phenomena of ontogenetic adaptation within each ecosystem supplies the basis for writing a set of local principles of adaptation $\left(\mathrm{L}_{1}, \mathrm{~L}_{\mathrm{H}}\right.$. etc.) for each species. Ecologically constrained generalization among the sets of local principles, as discussed in the text, produces a single set of global principles of adaptation $\left(G_{1}, G_{2}\right.$, etc.) that form the basis for a general theory of learning. Note that each set of local principles may contribute to more than one global principle and that every global principle may not apply to all species studied. The numerical relationships between local and global principles (whether one-many, many-one, or both) depends in part on the dimensions along which generalizations are made (see text). In this approach, ecological considerations are intrinsic to the completed theoretical formulation. 
discuss three possible alternatives, which are not meant to be either exhaustive or mutually exclusive.

The first lies closest to the spirit of the ecological approach, as I have outlined it in this paper, and involves searching along common task dimensions, identifying principles of learning relevant to feeding, orientation, habitat selection, social behavior, and so forth. Each such task dimension defines, albeit crudely, an aspect of the adaptive relationship between animal and environment that is common to all those species in which it can be identified. Searching for global principles along task dimensions thus seems a profitable strategy for understanding learning in terms of adaptation, which is a cornerstone of the ecological approach.

The second strategy is more familiar and is associated with comparative psychology as it is traditionally practiced - namely to search within taxonomic groups, to identify mammalian, avian, reptilian, and other principles of learning. The justification for this strategy is well known: Animals that are descended from a common ancestor presumably share characteristics that reflect ancestral constraints on possible evolutionary divergence. However, if a search along taxonomic lines is to contribute to an ecological theory of learning, it is important that the first two elements of an ecological inquiry - namely task descriptions and the elucidation of local principles of adaptation - play a more important role than they do in traditional inquiry (e.g. Bitterman 1975). It is not sufficient merely to compare animals on an arbitrary array of learning tasks. Rather, the tasks used should reflect the real adaptive problems that the various species encounter in their natural environments.

Finally, the third approach involves searching within groups defined by habitat or general life-style that is, by evolutionary analogy rather than homology. Thus, for example, we might look for general principles peculiar to arboreal animals, carnivores, migratory species, or highly social animals. This again is a relatively familiar comparative strategy, the aim being to discover principles that reflect evolutionary analogies in the abilities (in this case learning abilities) of different animals that adopt similar life-styles. This approach is an especially important source of information on the nature of the selection pressures responsible for the evolution of particular kinds of learning abilities. Once again, it is important that the learning skills among which such general principles of analogy are sought be anchored in ecological task descriptions for the particular species of interest.

I feel that all three of these alternatives will have something to contribute to a final general ecological theory of learning, since they provide insights into three different kinds of generality that hold among animals: adaptive, phylogenetic, and ecological.

Ethology and the ecological study of learning. The study of animal behavior in relation to the natural environment is often viewed as characteristic of the field of ethology, although it is sometimes forgotten that a number of psychologists have also adopted an ecological approach in their studies of behavior (see Miller 1977 for examples). Both by training and by inclination, then, ethologists are well-equipped to make important contributions to an ecological approach to learning, as frequent reference to the work of ethologists in this paper demonstrates. The essential stage of task description is one where ethological research has already provided a substantial body of literature that can serve as the basis for the study of whatever learning may be involved in these forms of behavior. Many instances of learning, such as the development of bird song, habitat selection, orientation, and prey capture, have attracted the attention of ethologists, but such studies lack the conceptual unification that might lead to the formulation of general theoretical principles of broad explanatory power.

The psychological study of learning, while lacking in ecological content, has strongly emphasized the desirability of seeking such general explanatory principles. Perhaps because psychology established its hegemony in the field of learning theory at a time when ethologists were largely concerned with the study of "instinctive" behavior, ethologists have generally drawn heavily on psychological theories in their discussions of learning (e.g. Thorpe 1963; Lorenz 1965; 1969). Finding those theories lacking in various respects, ethologists have offered criticism, but little in the way of alternatives (e.g. Lorenz 1965; Hinde 1973; cf. Mader 1977). It is clear that the ecological approach to learning advocated here will best be advanced by a synthesis between the traditions of psychology and ethology in regard to the study of behavior.

\section{Concluding discussion}

The ecological approach to the study of learning that I have outlined in this paper offers a more radical alternative to general process learning theory than does the currently popular biological boundaries approach. The latter has served a most important critical function, but, for the reasons discussed above, it seems to have serious limitations as a true alternative to general process theory. Let me therefore summarize the most important characteristics of the ecological approach, both theoretical and empirical, as contrasted with the general process approach, on the one hand, and with the biological boundaries approach, on the other.

The essence of an ecological approach to the study of learning is the realization that animals learn, under natural circumstances, to perform certain specific kinds of behavior. These instances of learning constitute the natural phenomena that it is the business of learning theory, as a branch of natural science, to explain. Unlike the general process approach, the ecological approach makes no prior assertions as to the existence of general processes underlying these various instances of learning. It is the business of students of learning to discover such processes, if they exist, rather than to assume them as the basis for a program of research. While it differs from the general process approach in reserving judgment on the existence of general processes of learning, the ecological approach differs also from the biological boundaries approach in being specifically concerned with the search for general principles of learning. The ecological approach, while fully endorsing the biological bounda- 
ries criticism, supplements that criticism with an alternative theoretical framework, quite distinct from that of general process theory.

The ecological analysis of learning is carried out at three different levels. The first anchors the analysis to natural phenomena of learning by providing task descriptions of what it is that animals actually learn in the course of their development. Constructing a task description requires that we study animals under natural or seminatural conditions, rather than merely making reasonable guesses as to what animals actually learn; there are many cases in which such descriptions are already available in the literature. In some instances of learning, constructing the task descriptions may be just as difficult, and may require just as much ingenuity, as analyzing the processes whereby learning occurs - it is most important that the significance of this element of the ecological approach be properly appreciated.

Given a task description for a particular instance of learning, the analysis continues with an investigation of the various experiences that contribute to the development of the skill in question, and the ways in which these experiences have their effects. How such investigations are to be carried out depends, of course, on the particular instance of learning being examined. Several exemplary studies are available in the literature; some of these are described above. Developmental analysis of this kind provides the basis for formulating local principles of adaptation that explain how individuals of a particular species learn to cope with the requirements of their particular environment.

The insistence that we formulate local (speciesspecific) principles of adaptation as a prelude to the search for more general principles is an important difference between the ecological and general process approaches to learning. An equally important difference between the ecological and biological boundaries approaches, both of which see a need for ecological study of particular species, is that the ecological approach advocates a third level of analysis: the search for global principles of adaptation that apply to more than a single species. This difference arises because whereas many biological boundaries theorists despair of finding unity within the wide diversity of animals, the ecological approach seeks such unity in the complementary diversity of animals and their natural environments.

\section{ACKNOWLEDGMENTS}

I am very grateful to M.-F. Cheng, G. Gottlieb, W. G. Hall, D. B. Miller, R. W. Oppenheim, and A. T. Pietrewicz for valuable discussions of these ideas and for comments on earlier drafts of the manuscript. The suggestions of the journal,s referees were also most helpful. This paper was completed while I was a Visiting Scientist at the Institute of Animal Behavior, Rutgers University; I thank J. S. Rosenblatt and C. G. Beer for providing that opportunity, and the participants in the Seminar on Animal Learning for helping me to clarify my thinking on a number of points. Preparation of the paper was supported in part by Research Grant No. HD-00878 from the National Institute of Child Health and Human Development.
NOTES

1. The points to be discussed do not represent original insights by modern authors. They were well appreciated by Darwin (1859) and by most subsequent evolutionary theorists. However, they are commonly not appreciated in less rigorous discussions of adaptation, and the authors cited must be credited for explicitly stressing their importance.

2. I should point out that the view I am expressing - that the components of an ecological theory of learning will be principles of adaptation - is not to be confused with recent proposals that the mechanisms of learning are analogous to the mechanisms of natural selection, whereby adaptations arise in the course of evolution (Staddon \& Simmelhag 1971; Campbell 1974; Staddon 1975; Glassman 1977). Whatever the merits of that view, it imparts no ecological content to the study of learning, and it has no bearing on the arguments at hand.

3. Not all solutions to behavioral problems necessarily involve learning, and a better formulation of the question might be: "How, if at all, does the animal learn to solve these problems?" It behooves the student of learning to select problems for study that are likely, on the basis of collateral evidence, to involve learning of some kind. However, it is also well to recall that many apparently unlearned behavior patterns, such as pecking by gull chicks (Hailman 1967) and the following reaction in young ducklings (Gottlieb 1971), turned out on close inspection to involve significant experiential components.

4. These three kinds of effects are distinguished by Gottlieb (1976). Maintaining experiences are required to support continued development of a pre-existing skill. Facilitative experiences enhance the development of a skill. Inductive experiences are those in the absence of which the skill does not develop at all.

\section{Open Peer Commentary}

Commentaries submitted by the qualified professional readership of this journal will be considered for publication in a later issue as Continuing Commentary on this article.

The next issue [BBS 4(2) 1981] contains two treatments of related topics: Plotkin \& Odling-Smee: "A multiple-level model of evolution and its implications for sociobiology" and Ghiselin: "Categories, life, and thinking.

\section{Principles of learning and the ecological style of inquiry}

\section{Thomas R. Alley and Robert E. Shaw}

Department of Psychology, University of Connecticut, Storrs, Conn. 06268

We applaud Johnston's advocacy of an ecological approach to the study of learning and a search for general principles. Moreover, his three-part categorization of approaches to learning theory seems, on the whole, to be both fair and useful. Despite our fundamental agreement with Johnston's position, however, there are a few aspects of his paper that we believe are in need of amendment.

We will focus on two of these issues in this commentary. The first issue concerns the relative importance, from an ecological perspective, of specifically understanding how and why animals learn. Second, we would like to emphasize and clarify an indispensible but often neglected characteristic of 
an ecological approach: the symmetry of descriptions entailed by the fundamental postulate of organism-environment mutuality (Shaw \& Turvey in press; Turvey \& Shaw 1979).

In light of the fact that Johnston advocates a search for general principles of learning from an ecological viewpoint, it is surprising that he declares "the question of central importance in the ecological approach" to be "What do animals learn and how do they learn it?" (emphasis added). If (as an ecological approach demands) learning is to be understood in terms of adaptation - in terms of compatibility of an animal and its natural surroundings - then the central question should be "Why do animals learn what they learn?" While both questions require a descriptive analysis of what animals learn, the question of how they learn it focuses on the processes of learning, whereas the question of why they learn what they do focuses on the adaptive significance.

As Johnston and the "biological boundaries" people have argued, there is reason to doubt the existence of general processes of learning. On the other hand, that every (speciestypical) animal is adapted to its (natural) environment is a fundamental tenet of modern evolutionary biology (Leigh 1971; Lewontin 1978). Given that a certain degree of fitness holds between every animal and its natural environment, the relationship of learning to fitness should be a prime target of the search for general principles of ecologically-relevant learning. Indeed, some very general principles of why animals learn are already known. Learning, for example, is more likely to contribute to behavioral patterns characterized by a high degree of variability in the relationship between stimulus conditions and the appropriate response (as in much foraging behavior) than when there is a reliable relationship between a stimulus pattern and a single adaptive response (as in most intraspecific recognition). Generally speaking, learning is associated with long life spans, prolonged and intense parental care, small litter size, large body and brain sizes, and slow development (Gould 1977; Mayr 1974). In sum, learning is (and from the viewpoint of evolutionary theory should be) associated with the life-history strategy known as K-selection, which means that learning will tend to play a significant role in contributing to an animal's fitness to the extent to which the species is subject to density-dependent selection in its environment of evolutionary adaptedness (Gould 1977; MacArthur \& Wilson 1967).

An example of the second aspect of Johnston's paper that we would like to amend occurs when he speaks of learning as contributing to "an animal's adaptation to its environment" (emphasis added). That learning is, on the whole, a form of adaptation, is one of the fundamental principles of an ecological or evolutionary approach to learning. But an animal cannot, strictly speaking, be said to adapt to its environment when "environment" is understood to be "those aspects of the ... world with which an animal interacts. - An animal may be said to adapt to its surroundings, but this process entails change in those aspects of the world with which it interacts. The "environment" of an animal, in the sense of its effective environment, will itself be altered by learning (and by other forms of adaptation). Indeed, a good way to characterize the process of adaptation may be in terms of changes in the way in which an organism interacts with and relates to its surroundings.

A related weakness occurs when Johnston mentions the "compatibility" between organisms and environments without emphasizing that this is a mutual compatibility: organisms are fit for their natural surroundings, and the surroundings are fit for certain types of organisms. This mutual "fit" or compatibility of organism and environment is the source of a fundamental postulate of an ecological style of inquiry - a postulate referred to by Shaw $\&$ Turvey (in press) as the "Postulate of Reciprocal Contexts," for the mutual fit of organism and environment is a reciprocal relationship ing that each be a context for constraining variations in the other. Although Johnston has endorsed this symmetry postulate (cf. Johnston \& Turvey 1980), his discussion of it in this paper (see "Global principles of adaptation") is so brief as to I be possibly misleading. The issue at stake concerns the fact that the role symmetry postulates play with respect to general principles is analogous to the role played by general principles with respect to processes. Just as general principles (of learning) are invariant over diversely embodied processes, so are symmetry postulates invariant over general principles or laws.

That symmetry postulates provide a higher-order context of constraint than general principles and, hence, logically condition their form, has been accepted in science since Einstein made symmetry postulates, rather than laws (general principles), the cornerstone of relativity theory and the arbiter of what form laws should take (Clark 1971; Wigner 1967 Whereas Newton saw symmetry conditions (conservation principles) as guaranteed by natural laws, Einstein saw it the other way around; the validity of laws were guaranteed only when conservation principles held, and conservation principles were guaranteed only when certain symmetry conditions held. For Newton, mass was defined in terms of force $(\mathrm{F}=\mathrm{MA})$. From this formulation Newton expected that the conservation of momentum would fall out (since momentum is defined in terms of mass times acceleration). Unfortunate. $\underline{\underline{l}}$, although momentum under this guise is conserved in many conditions, it is not conserved for high velocity collisions. To preserve the conservation of momentum, mass needs to be reformulated in accordance with a higher-order 1 symmetry postulate. As is well known, Einstein furnished such a reformulation at the level of space-time, wherein momentum is defined as an invariant vector quantity over all 1 frames of reference (Taylor \& Wheeler 1966). Einstein $r$ turned the world of Newtonian physics on its head; instead of formulating momentum in terms of laws, he formulated the laws in terms of momentum considerations (conservation) and $t_{t}$ momentum considerations in terms of symmetry over frames $t$ of reference. By doing so, Einstein rid physics of those 1 mysterious entities called "forces" with which even Newton was unhappy.

Returning to learning theory, our point is this: There is a strict analogy between learning processes and events (in Einstein's sense), for both are classes governed by laws of interaction. Similarly, there is a strict analogy between general principles of learning and the laws of physics, for both are governed by conservation conditions: fitness and adaptation in the case of learning principles, and conservation principles in the case of physical laws. The deepest constraints, however, are symmetry postulates. Here the analogy is between the postulate of organism-environment mutuality (the aforementioned "postulate of reciprocal contexts") of ecological science and the space-time invariance postulate of physical science.

Johnston, in stating that learning seen from an ecological perspective is "a process that ensures correspondence between an animal's behavioral skills and the requirements of its natural environment, - seems to have put the cart before the horse. Because symmetry postulates are the guarantors of general principles, learning principles must, from the ecological perspective, be seen as being guaranteed by adaptation (a conservation condition), and adaptation must be seen as being guaranteed by a deeper symmetry relation that necessarily holds over ecosystems: that postulated by organism-environmentmutuality (Animal 0 Environment). Under this reformulation of the problem, learning, like perception and action, is construed as an activity of an ecosystem rather than as an activity by an animal in an ecosystem (Turvey \& Shaw 
1979).

In sum, we believe an ecological approach to learning should, in its descriptions, concepts, and principles, strive to maintain the inherent symmetry that exists between organisms and their environments. Taking this approach, such misleading asymmetrical conceptions as that of an organism adapting to its effective environment or econiche may be avoided.

\section{A theory of learning - not even déjà vu}

\section{George W. Barlow and Stephen E. Glickman}

Departments of Zoology and Psychology, University of California, Berkeley, Calif. 94720

There is an admirable and indisputable set of principles presented in the Johnston paper: (1) The study of learning should proceed from an analysis of the natural contexts in which learning occurs; (2) Our views of the significant plasticity of behavior should not be circumscribed by allegiance to a too-narrow definition of learning boundaries as set by a few laboratory paradigms; and (3) A view of learning that consists, on the one hand, of traditional general-process theory and, on the other hand, of a set of exceptions to the rules, is not viable. From this point on, however, Johnston's article poses some difficulties.

The fundamental problems result from Johnston's attempt to establish the unique aspects of his own position by not giving sufficient credit to the thoughts of his predecessors. It is true that psychologists who are interested in learning have been slow to move toward naturalistic field observation. It is also true that some evolutionary biologists have by-passed the intricacies of ontogenetic processes in behavior and moved too quickly to outcomes. But it is no accident that many of the exemplary cases of behavioral analysis presented here come from biology. The three-level approach that is advocated boils down to the what, how, and why that pervades evolutionary biology. And it is important to stress that the founders of ethology were first broadly trained biologists who then turned their attention to animal behavior, applying the very approach advocated by Johnston, though ethologists are barely mentioned in Johnston,s article. Lorenz and Tinbergen stressed repeatedly the need to make empirical observations in nature and to formulate questions about processes in an adaptive framework.

Now if it is correct that Johnston adopts the approach of evolutionary biologists (read "ethologists") as the way to discover a unifying set of principles of learning, then it is difficult to understand how ethological "studies lack the conceptual unification that might lead to the formulation of general theoretical principles of broad explanatory power" (we do not, incidentally, agree with the quoted, overly broad, conclusion).

Actually, Johnston does not go far enough. He should present a more thoroughly biological stance. He brings along intellectual excess baggage. This is most evident in his treatment of learning as a trait that exists in an environment almost by itself. It would be far better to regard learning as a facet of an integrated behavioral system, as ethologists have done (see also the comments on Baupläne by Gould \& Lewontin 1979, cited by Johnston). Johnston's approach allows one to write about simply going out and observing learning as it occurs in nature, when in fact a central question is the extent to which experience and genome are involved in the elaboration of any behavior. There is, nonetheless, a wealth of biologically relevant phenomena that are adaptive in the immediate, learned sense, such as decision making while foraging, the acquisition of cognitive maps of territo- ries, and the identification of individuals. A completely neglected area of research is the genetics of learning.

Johnston mentions the comparative approach almost in passing. It deserves more attention because it contains the recipe Johnston seems to be seeking, and because "comparative" has meant such different things to psychologists and evolutionary biologists. Biologists analyze phyletic lineages, seeking to establish which traits are primitive, and which are derived; there is a recognized methodology that has proved effective despite the difficulties involved. The more primitive, the more general. It is precisely this sort of programmatic attack that can answer questions about the generality of processes and principles.

The complementary approach is the truly ecological one. It involves a careful search for correlations between ecology and behavior, followed by hypothesis testing. The ecological variables include demographic considerations together with distribution of, and access to resources (most of the time, Johnston uses ecology when biology is closer to his meaning). Such a two-pronged undertaking would clarify which aspects of learning are due to shared ancestry (primitive, homologous) and which are due to convergent or divergent evolution (derived, analogous). Questions about adaptiveness would then seem less formidable.

The concept of adaptiveness troubles Johnston. The difficulty, as we see it, is that a trait is designated as adaptive or not adaptive (though it might also be neutral), which is pretty much an either/or way of thinking. The concept of relative fitness, estimated in terms of the number of offspring left in the succeeding generation, offers a better means for understanding the situation, though just how one actually measures fitness is not as simple as had been hoped. Nonetheless, fitness has the advantage of varying over a range of unfit to highly fit. The essential point, however, is not whether the behavior is adaptive, or whether it affects fitness, but simply whether it is a normal part of the animal's life, with an identifiable function (task description).

The laboratory learning psychologists have also made a set of contributions which still have significance for the problems. It is precisely because psychologists have identified critical variables that literatures have developed in the fields of bait-shyness, bird song learning, and imprinting, and that they have had such an impact. Moreover, one can enrich the study of learning or plasticity without discarding examination of the nature of reinforcement, the timing of conditional stimulus-unconditional stimulus (CS-US) intervals, the role played by overt responding in the learning process, and the like.

As regards the ad hoc nature of many recent attempts to assign adaptive significance to laboratory observations, Johnston again has a point. But he moves too quickly. The Shettleworth (1975) study is a notable example. Shettleworth discovered that food reinforcers modify the rate of emission of some . operants" but not of others. It is significant that she did not stop at ad hoc speculations relating her results to the presumed natural situation. Rather, she deprived hamsters of food and observed the ensuing changes in the rate of emission of these particular operants. Her results were consistent with the idea that food-related operants were better controlled by food reinforcement. This was not observing behavior in nature, but it was not simple ad hoc reconstruction either.

The biological approach to the study of learning has the potential of making an important contribution to evolutionary biology. Evolutionary theorizing has tended, of late, to ignore behavioral processes and to concentrate instead on the outcomes of behavior. The efforts of animal behaviorists may be required to direct attention back to the evolution of the mechanisms themselves. Learning phenomena provide excellent material for a thoroughly evolutionary approach to the 
analysis of behavioral processes and could bring fresh insights to the interplay of short-term (ontogenetic) and long-term (phyletic) adaptation. In this sense, Johnston's article is an important contribution.

Progress in well-established fields is sometimes saltatory, but more often it is an almost imperceptible rearticulation of existing findings and points of view. A given essay may seem to differ little from its predecessors. Yet, after a decade of such articles one can look back and perceive an important change. Johnston's paper is presented as a major departure from existing work in the field; it is instead a rearticulation. Although the present paper is not as novel as one might wish, it is in line with a significant move toward placing the study of learning in an appropriately biological perspective. There is room, however, for a still more radically biological approach.

\section{Linking the biological functions and the mechanisms of learning: Uses and abuses}

\section{Patrick Bateson}

Sub-Department of Animal Behaviour, University of Cambridge, Madingley, Cambridge CB3 $8 A A$, England

As an ethologist, I am fond of speculating about the functional significance of behaviour. Looking at animals in the conditions to which I think they are adapted helps to refine the ideas. Some possibilities leap out of the bushes, so to speak, while others that seemed so plausible in the armchair at home are hastily rejected. To go further requires experiments of the type that were so elegantly pioneered by Tinbergen (Tinbergen, Broekhuysen, Feekes, Houghton, Kruuk, \& Szulc 1962). All this is very much in tune with the first part of the programme that Johnston has sketched out. However, the second part, in which functional principles are to be translated somehow into an understanding of mechanism seems much more obscure to me. On the face of it, the translation is impossible. Would an ecological theory that told us how an engine could be adapted for use in motor bicycles, cranes, pumps and chain saws, give us any insight into how the internal combustion engine works? Does the ecological theory of mating systems in birds and mammals (Orians 1969; Emlen \& Oring 1977) allow us to predict (if we did not know already) whether or not the ducks have an organ of intromission? I thought that Johnston would avoid the logical fallacy when he made the useful distinction between principle and process. But then he went on to blur the issue by arguing that a functional principle can be used to explain the character of the mechanisms. For all that, Johnston may be right in saying that one approach helps the other, even though he has given the wrong reasons for why it might do so. I believe that an understanding of the adaptedness of a behaviour pattern can help us indirectly to understand how that form of behaviour is controlled. Functional explanations are commonly expressed in teleological terms. Biologists protest, of course, that the teleology is merely a shorthand, but they implicitly make the point that the language is actually helpful. Since we are all enthusiastic planners of our own lives, thinking of the outcome of an animal's behaviour as a goal can help us get our minds round the complex processes that lead to that outcome. So the teleology can provide us with a means of thinking about the whole system.

A different point is that in examining what Johnston calls the task description, we are alerted to the conditions in which the character of behaviour changes. Knowledge of those conditions is crucial when we start to design experiments in which inevitably only a small number of independent variables are actually manipulated, while the others are held constant or randomised. The experiment is a waste of time if the parameter values that are not subject to explicit manipulation are badly chosen. This is the relevance of the frequently stated point that we need to know our animals before we can do anything really interesting with them.

It was on the issue of developing an adequate knowledge of learning that Johnston did not really do justice to the so-called biological boundaries theorists. Admittedly, the terms "boundaries" and "constraints on learning" are unfortunate. Indeed, at the end of a conference that was later turned into a well-known book (Hinde $\&$ Hinde 1973), there was considerable discussion about what the book should be called. I, for one, argued against the use of the word "constraint" in the title because I felt that the whole notion lay too comfortably in the framework of existing learning theory. It does not adequately stress the active behavioural processes that predispose learning to occur in particular contexts (Bateson 1973, p. 102). As a compromise, the members of the conference agreed to the use of "predisposition" in the subtitle. That was not enough, as it turned out, and the general process theorists, like Revusky (1977), could easily dismiss the constraintson-learning approach as nit-picking and obscurantist. Mind you, if Revusky really believes that asking grand questions necessarily guarantees the importance of the science, he should think again. Pretentiousness is not the same as quality. In any event, the rear-guard rhetoric was not directed at the most damaging criticism of classical learning theory. It was not simply that the theory was wrong, but that the approach that generated it was misconceived. The grand theorists were attempting to run before they could walk. The case for a more open-ended approach to the study of learning is not that it immediately produces a counter-theory, but that it provides a much more secure basis on which to formulate general principles. This was a major message of Hinde's (1973) critique. Inasmuch as Johnston echoes that message, he is very much a part of the company of thinkers whom he claims to be superseding.

\section{An ecological approach toward a unified theory of learning}

\section{William R. Charlesworth}

Institute of Child Development, University of Minnesota, Minneapolis, Minn 55455

Johnston's paper is important; it is refreshing and distinctive It starts off, however, with an unfortunate weakness. Its title should have begun with "An ecological approach ....." rather than with "Contrasting approaches for the paper's significant contribution to research in learning is the ecological perspective, and it would be very unfortunate if potential readers passed over this because they have already read too many comparisons of learning theory.

Those who are better informed about learning theories will. of course, find points to pick apart, but this paper is less a comparative analysis than it is a pioneering thrust. Pioneers do not have to elaborate fine points; they do, however, have to go in the right direction. Johnston does. He offers an exciting expansion of learning research as part of a wider ecological revolution that is gradually building momentum in various behavioral sciences. Examples of this revolution are the late James Gibson's (1979) imaginative views on visual perception [see also Ullman: "Against Direct Perception" BBS 3(3) 1980]; McGurk (1977) and Bronfenbrenner's (1979) efforts in developmental psychology; Sackett,s (1978) thrust to expand observational research in mental retardation; and Shaw and Brans. ford,s (1977) interesting collection of papers covering a wide range of psychological topics. 
The approach presented here actually has more roots in comparative psychology and classical ethology than those mentioned by Johnston. Wesley Mills (1898), a comparative psychologist and naturalist, was an early proponent of field research. His approach contrasted with what he termed "mere closet psychology," which was widely practiced at the time in armchairs and laboratories.

In classical ethology, a phylogenetic version of the ecological approach was foreshadowed in Konrad Lorenz's (1941) paper, which viewed Kantian thought categories in the light of evolutionary biology. Lorenz's thesis was that the cognitive apparatus of animals represented an evolutionary adaptation to external environmental conditions present during phylogenesis. This thesis emphasized the phylogenetically acquired dimensions of cognitive function, as well as the evolution of learning abilities that make possible individual adaptation to idiosyncratic conditions during early ontogenesis; the latter emphasizes the importance of environmental factors in directly shaping behavioral ontogeny. Indeed, the whole imprinting literature deals with the attempts of young animals to take advantage of local social and physical resources for survival purposes, and with their being significantly changed as a result of those attempts. Immelmann's (1975) paper on the relationship between ecological factors, imprinting, and early learning expands on this thesis both by citing research literature on "ecological imprinting," which leads to the establishment of food preferences, home area selection, habitat preference, and host selection in parasites, and by speculating on the relevance of environmentallyinduced modifications for speciation. [See also Rajecki et al.: "Toward a General Theory of Infantile Attachment" BBS 1(3)19781 So it is not completely true that ethologists have, as Johnston claims, offered little in the way of an alternative to psychological theories of learning. The clearest and most conceptually elegant formulation of an ethological/evolutionary view of learning can be found in Ernst Mayr's (1974) paper on genetically "open and closed programs.

Johnston,s claim that the biological boundaries approach is methodologically subservient to the general-process tradition seems correct, but he undermines his claim by enlisting "modern philosophers of science" who argue that there is nothing like a conceptually neutral methodology. If we define methodology as a manner or mode of procedure (as is done in standard dictionaries), then it is difficult to see how methods per se can be anything else but neutral. What is not neutral is the decision to choose one method over another. Theoretical predilections, personal biases, etc. influence choice of method, but, once chosen, the method neutrally runs its course. There are at least two important reasons why field research is chosen less often than lab research: The first is convenience (ever try following a goose all day to watch it learn?); the second is publishing time (ever try to turn out a sufficient number of good observational studies to meet the annual demands of an institution's reward system?).

Johnston,s point that much research on learning emphasizes the logical rather than the ecological structure of tasks is well taken. The same point can be made of most psychometric research on human cognition and intelligence. Piagetian research, as Brainerd (1978) points out, is a good example. Aimed at studying the cognitive products of individual/ environmental interaction (a general learning process), such research focuses almost exclusively on the logic of cognitive structures. With the exception of Piaget's own classical observational research with his three infant children, there has been little serious empirical concern in Piagetian circles for the ecological representativeness of the test items used to tap cognitive structures or the possible adaptive value of the cognitive structures measured. [See also Sternberg: "Sketch of a Componential Subtheory of Human Intelligence" BBS 3(4) 1980.]
Johnston has pushed us a significant step closer to a unified theory of learning. This unified theory will be an updated, expanded version of what Darwin actually had in mind for behavior in general. As Reed (1979) so aptly argues, Darwin's insight was synoptic enough to include within the same theoretical framework proximate causes as well as selection pressures and their consequences. Learning has form, cause, and function; it takes place inside an organism that is interacting for very important reasons with an environment; and both the organism and the environment have short and long histories of great relevance for our understanding of adaptation. Now that we have got that settled, let those of us in different disciplines get our separate acts together. It is the only way that makes sense.

\section{The nature of learning explanations}

\section{John Garcia}

Departments of Psychology and Psychiatry, University of California, Los Angeles, Calif. 90024

Johnston divides all learning theory into three parts: A, the ecological approach; B, the biological boundaries approach; and $\mathrm{C}$, the general process approach. To set up B, he cites Garcia \& Koelling (1966) and Garcia, Ervin \& Koelling (1966), and then lists three criticisms indicating why B is inferior to $\mathrm{A}$ in the historic movement away from $\mathrm{C}$. These criticisms are (1) "the biological boundaries approach lacks generality, ... (2) it is in some respect subservient to the general process tradition, and (3) its ecological content is in too many cases limited to ex post facto adaptive explanations of learning skills." Furthermore, we once wrote a paper entitled "Biological constraints of conditioning" (Garcia, McGowan \& Green 1969), which was reprinted by Seligman \& Hager (1972) in Biological Boundaries of Learning and cited by Johnston, who wrote, " . . the very use of terms like 'boundaries' and 'constraints' implies that something (a general process?) is being constrained. - Admittedly, we should have watched our language, but I respectfully submit that if the content of our papers is examined, it will be obvious that we were on approach A.

To protest (1), lack of generality, I point to two papers. First, Garcia et al. (1966) indicated that taste is by far the most effective conditional stimulus (CS) for a poisonous unconditional stimulus (US), particularly when the US is delayed for a half hour or more. We referred to the natural niche of the wild rat and pointed out that temporal and spatial parameters of conditioning depend on what the rat is learning to do. Soon after, Garcia and Ervin (1968) argued that the poisonous effect possessed great generality, noting that the taste CS and the noxious visceral US converged via neural routes to the same relay station in the medulla oblongata of the salamander, an arrangement that is apparent in a wide phylogenetic array of species, including man. Rapid taste-poison learning has been demonstrated in many related species; it has also been demonstrated in unrelated species, such as the mollusks, which may have acquired similar mechanisms through convergent evolution, because toxic plants are prevalent throughout the earth (Garcia \& Hankins 1975).

To protest (2), subservience to general process, I quote from our "constraints" paper (Garcia, McGowan \& Green 1969): "It is our task to devise hypotheses from field (naturalistic) observations and to design (laboratory) experiments ... where the relevant ecological variables could be experimentally manipulated." We belabored approach $\mathrm{C}$ because we were told that it did not matter whether we studied pigeon, rat, or monkey (Skinner 1959), and that there was no need for 
neurophysiological specification (Spence 1947). Spence substituted the "black box" for the behaving animal, and Skinner searched for his general laws among the environmental interactions surrounding the black box. It is ironic that Johnston begins by giving us an illustration of a "general principle" in the Skinnerian mode. His equation $\left(\mathbf{Q}_{\text {prod }}+\right.$ $Q_{,,,}-Q_{\text {loss }}=0$ ) cannot distinguish between a panting dog and a sweating human, much as Skinner's scallops, shaped by a fixed-interval (FI) schedule, could not distinguish a pecking pigeon from a manipulating monkey.

We went farther down approach $A$ than Johnston himself when we said "Evolutionary explanations invoking natural selection are aversive to most psychologists because ... the evolutionary determinants appear to be lost in history and cannot be directly verified. But the end product of natural selection exists in the anatomical structure of the organism and there, theoretical learning propositions can be independently tested" (Garcia, Palmerino, Rusiniak \& Kiefer 1980, in press). Johnston stops short, offering us half of approach A. He begins by discussing the "natural environment," then, belatedly and parenthetically, he mentions that behavior must be specified, ". . (ultimately, though not necessarily immediately, in physiological terms)" (emphasis added). Johnston is pushing a new line of "ecologically designed" black box models with a rebate, a promissory note, to be paid off in physiological currency at some vague future time.

To protest (3), ex post facto adaptive explanations, I say, "better late than never, but they must be neurophysiologic and phylogenetic ones. - This is the only way we can escape the circularity of interpreting behavior with more behavior, as Johnston does when he explains, ... . avoidance of noxious foods is mediated by different cues in different species, ... . determined by the way in which food is typically selected by each species. - In fact, when poisonous effects are delayed, learning appears to be mediated by taste in both mammals and birds, as if the taste-visceral, neuropil contains an "andgate" which allows other cues to become food cues when they are contiguous with taste. Even a taste that by itself appears to be an ineffective signal for a given poison dose can potentiate a weak cue into an effective one. (For a review see Garcia \& Rusiniak 1980; Garcia, Palmerino, Rusiniak \& Kiefer 1980).

A superabundance of approach $\mathrm{C}$ experiments have shown that, with appropriate training procedures, animals can be taught to avoid a place where they have been ill, or to drink more fluid in a safe place than in a place where they became ill. These data are difficult to reconcile with the taste-visceral hypothesis because the sensory inputs are rarely isolated, and the neural routes are not even suggested. The potentiation phenomenon makes some of these experiments even more difficult to interpret, but that is the concern of approach $\mathrm{C}$.

I confess that I am one of those who still believe " . that 'facts' exist to be 'collected' by a convenient methodology, like collecting pebbles on the beach. - Bulldozing the entire behavioral beach and sifting it into an orderly array of grains, pebbles, rocks and boulders of adaptation is too much like work. I'm glad to learn that "this view has been almost universally abandoned, with good reason, by modern philosophers of science. - We need them about as much as poets need literary critics.

\section{The relevance of phylogenetics to the study of behavioral diversity}

Michael T. Ghiselin

Department of Biology, University of Utah, Salt Lake City, Utah 84112

I would like to reformulate the problem of biological boundaries versus general-process approaches in the light of taxo- nomic theory. Everybody seems to agree that evolution is important here, but they do not agree on what to do about it. Much of the trouble may stem from efforts to treat psychology as if it were a kind of physics rather than a branch of biology. Behavior is viewed as something static, and the history of an organism's lineage is viewed as a mere annoyance. The attempt to model psychology upon physics is particularly apparent in the work of Hull (1945), who hoped to write equations for the general processes using "empirical constants" to get rid of the embarrassing load of particulars.

Such approaches to behavioral diversity evidently involve searching for generalizations that are called, perhaps misleadingly, "laws." The data that complicate the application of these laws are taken to be the result of special conditions, to be dealt with from a naively teleological point of view. It is assumed that the organisms are adapted to their environment without the qualifications that follow from what is known about evolution. The addition of more "laws" about what is "good" in each environment would allow one to screen out the diversity as so much irrelevant "specialization." But, as Johnston points out, there are certain ancestral constraints that pose serious difficulties.

It is most unfortunate that the generalizations of taxonomy are so often confounded with laws of nature. The diagnoses of taxa are statements about known historical fact. They are a different kind of generalization. What is universally true of the learning of mammals might be true because some law of nature necessitates that all learning will have certain properties. It may also be true because all mammals live under those conditions where the law applies. Or it may be true because it was true of the common ancestor of mammals and the descendants did not change.

In principle, at least, we can construct a phylogenetic history of the various features of all organisms. Some of those features have recently evolved and tend to be less widespread than others. Perhaps in the history of animals the basic mechanisms of learning go very far back, are monophyletic, and have not changed. Other mechanisms of learning, such as color vision, seem to be later, polyphyletic acquisitions with secondary losses. Thus the rats studied by Galef and Osborne (1978) not only have a long history of not responding to the appearance of food because they were fed in the dark, but they, as well as numerous other diurnal feeders, are also color-blind. It would seem that color vision is not readily acquired, even in niches where it would have great survival value. Organisms are not adapted to their environments simpliciter. They do, as their ancestors did, whatever happened to maximize their ancestors' reproduction, in what happened to be their ancestors' environments, with whatever happened to be their ancestors' legacy to them.

The existence of diversity in a range of organisms helps us to sort out the interplay among history, environment, and in by using phylogenetics. A straightforward example of such a study in psychology is Darwin's work on climbing plants (Darwin 1876 and earlier versions). The faculty of climbing I has evolved several times. Plants climb by using severs' 1 different organs: stem, shoots, petioles, and even roots. Which organ gets used is a characteristic of species and higher taxa as well. The diversity shows that what gets used in climbing is not very general and results, in part, from historical accident Yet Darwin,s physiological research did reveal some more widespread general principles, such as a circling movement. Some features of behavior ought to be common to both plants and animals; they might even evolve in extraterrestrial beings. I cannot agree with Smart (1963) that there are no biological or psychological laws. I do suggest, however, that these laws would have to be something other than generalizations about a single species, genus, or other taxon. The way to find laws is through the study of such convergences as have appeared in the evolution of vertebrates and cephalopods. 
Johnston: Learning theory

But one cannot know that convergence has happened unless one knows the actual phylogeny of the organism.

The historical aspect of adaptation is all too often overlooked. As I have pointed out (Ghiselin 1966), there is adaptation as a state, adaptation as a process, and adaptation as a state resulting from that process [see also Ghiselin: Categories, Life, and Thinking" BBS 4(2) 19811 The mere fact that a benefit seems to accrue from a feature does not suffice to demonstrate that it is an adaptation in the sense that it was produced by selection. To do that, one needs to study the historical record. If one can show that a series of modifications began to be produced when the ecological circumstances and selective regimen changed, one has compelling evidence that adaptation has occurred.

\section{On the what and how of learning}

\section{R. C. Gonzalez and Matthew Yarczower \\ Department of Psychology, Bryn Mawr College, Bryn Mawr, Penn. 19010}

Johnston,s outline of what he terms an "ecological approach to the study of learning" is actually an outline of an ethological approach to the study of animal behavior, which leaves room for the study of learning, but gives no hint of how the processes of learning are to be elucidated.

Johnston argues, in effect, that behavior theory should be developed in relation to the natural environment of organisms, with special reference to what animals actually do under natural circumstances. "Local" principles of adaptation are to be identified to account for the behavior of individual species, and commonalities among these species are to serve to establish general principles of behavior.

Johnston's criticism of the "biological boundaries approach" (with its reliance on ex post facto adaptive explanations and its inherent pessimism about common processes) is well taken. His treatment of the learning issue in general, however, leaves much to be desired. He fails, it seems, to grasp the distinction between a theory of learning and a theory of behavior and to see the relation between the two. Theories of learning are directed to the understanding of what organisms learn, that is, the products of learning (e.g., associations and their nature, relations among environmental events and their representations, etc.); of how they learn, that is, the necessary and sufficient conditions of learning (e.g., contiguity, contingency, effect, etc.); and of how learning is transformed to performance, that is, the processes that translate the products of learning into behavior. Theories of behavior are much broader in scope. They include learning postulates among a wide array of other determinants of behavior (e.g., perceptual, motor, motivational, maturational, instinctual, etc.) and must deal with the interrelations among the various determinants.

When Johnston sets out to answer the question "What does this animal learn to do?" he has already set aside a major issue in learning theory by asserting that what animals learn is, in fact, to do. (Thorndike came to essentially this conclusion, but on the basis of his research. Other theorists, of course, disagree.) His six-point program for answering the question "How does the animal learn to do that?" is directed not to the issue of the conditions of learning, but rather to the various determinants of the behavior in question (including learning) in the broadest terms. It is difficult, therefore, for a student of learning to accept the proposition that Johnston is dealing here with "an approach to learning theory." And he is mistaken in his evaluation of some efforts in the comparative psychology of learning.

It is correct to note that, in experimental psychology, behavior theory has been dominated by learning theorists who, with a few notable exceptions, have tended to assume, in the manner of Thorndike, that the same processes of learning operate everywhere in the animal kingdom - that animals differ not in method of learning, but only in proficiency. It should be noted that Thorndike came to his conclusion on the basis of the results of his own experiments, which were designed to test this very proposition (Thorndike, 1911). Thorndike's failure to find objective evidence of qualitative differences in the modes of solution of problems posed to chicks, cats, dogs, and monkeys - despite marked differences among the animals in their rate of acquisition - led him to conclude that observed differences in behavior among animals are to be accounted for in terms of quantitative differences in the operation of the same processes of learning. In retrospect, it is easy to see that Thorndike simply lacked the technological facilities (readily available today) to carry out experiments that would adequately detect the operation of distinct processes. (Few of his results have stood the test of replication.) He was not limited, however, in his conceptual approach to the problem.

It is quite true that, in general, students of learning since Thorndike have adopted the Thorndikian conclusion as a matter of conviction rather than treating it as a hypothesis for experimental testing (and the result has been that their choice of subjects for research tends to be made rather cavalierly primarily in terms of convenience). However, it is not true, contrary to what Johnston implies, that a comparative psychology of learning is beyond the scope of the learning theorist's laboratory, or that there is some inherent limitation to analytical work done outside the natural setting. In his criticism of one such comparative enterprise, Johnston asserts that "It is not sufficient merely to compare animals on an (ecologically) arbitrary array of learning tasks. Rather, the tasks used should reflect the real adaptive problems that the various species encounter in their natural environments." But "learning tasks" (experimental contingencies) that seem to be arbitrary ecologically may be far from arbitrary in relation to the processes of learning that they engender, a fact that may require considerable sophistication in both the theory and the technology of learning to appreciate fully. Some of these "arbitrary tasks," in fact, have been the ones that have finally begun to yield objective evidence of the operation of qualitatively different processes of learning in mammals and nonmammals (e.g., Bitterman 1975; Gonzalez, Ferry \& Powers 1974; Gonzalez, Potts, Pitcoff \& Bitterman 1972; Pert \& Gonzalez 1974). To the extent that such results bear on anagenesis, they have clear evolutionary significance (Yarczower \& Hazlett 1977), despite the fact that the "arbitrary tasks" involved were not dictated by the ecological imperative.

It should be noted, too, in relation to the claim that laboratory work in the tradition of the learning theorist is sterile with respect to the understanding of behavior in the ecological niche, that behavioral principles (as distinct from theories of learning) derived from laboratory work on learning have proved useful in understanding phenomena in natural settings. Hailman (1969), for example, has made use of principles of classical conditioning to account for the development of the precise pecking by laughing gulls at the mother's red bill. And Hoffman and Ratner (1973) have brought behavioral principles derived from both classical and operant conditioning to bear on the analysis of the phenomenon of imprinting.

What, then, is there to be said about Johnston's approach? In our opinion, its contribution is its provision of an outline of a unified, coherent scheme of ethology whose merits (which may be considerable) are ultimately to be judged by its usefulness to investigators of phenomena in natural settings. It has little, if any, relevance to the development of a theory of learning. 


\section{Discussing learning: The quandary of substance}

\section{Jack P. Heilman}

Department of Zoology, University of Wisconsin, Madison, Wisc. 53706

Whenever I read an apparently sensible article like Johnston's, in which I can find nothing of substance to challenge, two possibilities occur to me: either the author's biases are totally coincidental with mine, or else the article has no substance. If the former be true, it is unlikely that I can fulfill the role of critic - or I would have done so long ago and consequently changed my views. The only course of action is therefore to see if a case can be made for the target article's lacking substance. I will do my best.

As this is a theoretical article, there are no data, apart from a brief summary of a few results from the literature, and there are no alternative interpretations of existing data. Johnston does not deny that general processes of learning exist; he merely separates processes from principles by definition. From this separation one might expect principles to be articulated, even if the processes cannot be, but no such articulation occurs. Johnston simply asserts that we need to know what an animal learns and how it learns it. By analogy with the example on thermoregulation, this is the equivalent of saying that one must measure the heat produced, gained, and lost; the general principle lies in stating that the sum of the first two must equal the third, but no such principle is forthcoming concerning learning phenomena. The author did not promise the reader any of these things, so that (apart from some very general orienting attitudes) the substance of the argument must lie in the details of the "what-and-how approach" to learning.

The relevant details appear to consist primarily of two points about adaptation, six questions relating to local principles, and three "dimensions along which we should search for global principles." Let us consider these in turn. First, the two points about adaptation are (1) that adaptation is a relation between animal and environment, and (2) that not all characteristics of an animal are necessarily adaptive. The former point is logically a restatement of the definition of adaptation, and the second is virtually self-evident — although I would admit that some biologists need convincing (Hailman 1977a). As to the exemplary six questions about local principles, the list differs in no substantial way from similar lists that have been in the literature for a long time (e.g., Hailman 1967, pp. 129-31). The remaining three "dimensions" are familiar strategies of research. The first, which is designed to show that a particular characteristic of an animal works admirably in given circumstances, is what C. Richard Tracy (Burtt 1979, p. 422) dubbed the "engineering" approach when he characterized my analysis of visual signals (Hailman 1979). Basically, the reasoning of the engineering approach is that if something does the job well then it probably evolved to do that job; this is so common a mode of approach that it is rarely explicitly emphasized. The other two points - correlating animal characteristics with taxonomy on the one hand and with environment on the other - are simply an incomplete statement of the comparative method familiar to all evolutionary biologists (see, for example, Hailman 1976 or $1977 \mathrm{~b}$, pp. 13-15 for the complete logic of the comparative method).

I can thus find nothing new in Johnston's viewpoint, except possibly this: if the article is aimed at psychologists who do not already know all these things, then everything is new, and indeed, quite substantial, in an important sense. If Johnston can persuade psychologists to study learning in a relevant biological context, then just possibly he or someone else might eventually pursuade biologists to insert learning phenomena into ethology and deliver us from the instinct-theorists in their latest guise of "sociobiology."

\section{Biological approaches to the study of learning: Does Johnston provide a new alternative?}

Robert A. Hinde

MRC Unit on the Development and Integration of Behaviour, Madingley, Cambridge CB3 8AA, England.

Any step toward continuing or extending the dialogue between psychologists and biologists interested in the development of behaviour is to be welcomed, and one hopes that Dr. Johnston's paper will stir up further debate. It is, however, not immediately apparent that it involves as big a step forward as he suggests.

Johnston is inaccurate in supposing that the learning theorists of the last fifty years believed that "all examples of learning are manifestations of an underlying general process." And he is wrong to suppose that a biological approach to learning (which he inappropriately calls the "biological boundaries approach") denies the existence of general principles of learning. Furthermore, most ethologist interested in these problems have had no intention of replacing or providing an alternative to the work of the learning theorists; they have attempted, rather, to marry the two approaches, specifying limitations to the earlier generalizations. Over these latter issues Johnston reveals a misunderstanding of the nature of biological principles - perhaps this is because he, like many philosophers of science, sees the biological psychologist as striving after something akin to the physicist's $\mathrm{e}=\mathrm{mc}^{2}$. Apart from the theory of evolution by natural selection, we are not as yet in sight of a master key to the phenomena of behavioural development, and we must seek for relatively short-range principles, each coupled with a statement about the range of its validity. The more precisely each principle is stated, the smaller the range of phenomena to which it will apply, but either to deny or to assert the existence of principles would be to miss the point.

Johnston's distinction between "task description" and how the task is achieved is similar to the distinction between the problems an animal has to solve and how it solves them. This is, of course, a commonplace in biology (e.g., Pantin 1951, 1952; Tinbergen 1951); indeed, the analogy of the engineer building a bridge, used by Johnston, was also Pantin,s favourite. But as Johnston points out, "task description" sometimes embraces quite a bit of analysis, and he uses it both for the problem the organism has to solve ("Bridge that river"; or "Steer in a given direction") and for the description of a solution ("Use a cantilever structure" or "Use the Pole star"). I find that the task description is difficult to distinguish from the functional description long emphasized as an essential first step by ethologists.

Johnston,s next phase is a phase of causal analysis, aimed at a description "ultimately, though not necessarily, in physiological terms," but in the short term involving precisely those questions that any ethologist would ask about development, that is, "What are the necessary and sufficient conditions for the behavior to develop?" "Is there a sensitive period for particular experiential factors?" etc. Again, I find it difficult to distinguish this analysis from what all ethologists interested in development have been doing for a long time.

Johnston's final stage involves searching for general principles that will embrace the answers to the "how" questions about particular examples of learning in particular species, in the light of the complementary diversity of animals and their environments. This is a laudable goal, and one toward which little progress has been made by either ethologists or comparative psychologists (however, see Schneirla 1949). But I find it difficult to see how it can possibly be an alternative to the work of learning theorists. The generalizations that emerge will be in functional terms and not in terms of "mechanism As Pantin pointed out, there are many different ways to build 
Johnston: Learning theory

a bridge: the generalizations that emerge from "global principles of adaptation" will be functional generalizations compatible with, but in no way replacing, the generalizations that emerge from learning theories.

\section{A funny thing happened on the way to comparative psychology}

James W. Kalat

Department of Psychology, North Carolina State University, Raleigh, N. C. 27650

Early comparative psychologists, especially prior to the 1920s, focused on comparisons of many species in many learning situations. Every species, from pigs to pigeons to porcupines, was tested for delayed response, alternation, maze learning, detour, and other complex responses (Maier \& Schneirla 1935). Most of these tasks would qualify as "ecologically arbitrary," to use Johnston's apt phrase, as the goal was to map out the phylogeny of intelligence, which was assumed to be a single entity. It was naively assumed that the learning abilities of animals could be rank ordered along a single dimension from protozoans to humans. There was little consideration of the animals' natural ways of life, except when it was necessary to modify the dimensions of the apparatus. J. 0. Cook (1953) remarked that this kind of research generally led to a "plethora of data and a paucity of theory.

When such great system-builders as Hull and Tolman superseded the early comparative psychologists, they investigated a single species - the rat - in a few situations. But while they abandoned early comparative psychology's method of interspecies comparisons and its interest in the phylogenetic distribution of intelligence, they remained faithful to the assumption that animals' learning abilities differed only quantitatively. In fact, this assumption was quite consistent with their exclusive attention to the rat. If learning is a single process, differing only in quantitative parameters across species and situations, then someone interested in its processes and mechanisms has no reason other than convenience to study one species rather than another.

T. D. Johnston's position represents an interesting reversal of the revolution that occurred in the 1920s. Instead of abandoning the method of interspecies comparisons but maintaining the assumption that intelligence is a single entity, Johnston argues that we do the opposite: we return to early comparative psychology's method of interspecies comparisons, but we abandon its assumption that all learning involves the same processes. However, we would restrict our comparison of species to studies of how they cope with real-life problems. In fact, Johnston is calling for the kind of comparative psychology that we should have established long ago - a field that relates the behavioral capacities of animals to both their evolutionary history and their adaptation to their natural environment, in a manner truly analogous to that of comparative anatomy and comparative physiology.

One example that Johnston gives is quite illustrative of the kind of generalization toward which this field could strive: "... we might find, for example, that the number of encounters (trials) that an animal requires in order to learn to avoid noxious food items is a function (possibly complex) of the harmfulness of noxious items, the relative frequency of noxious items, and the energetic costs associated with the capture of a single food item." If we ever reach the point of establishing statements of that form, we may come to regard the psychology of animal learning, as it has existed until the present, as a monumental detour on the way to comparative psychology.

I find that I am highly sympathetic to Johnston's position. As he remarks, it is in many regards similar to the position of
Rozin \& Kalat (1971). Where I differ from Johnston is in his insistence that his position is an alternative to both the general-process and the so-called "biological boundaries" approaches. The disagreement between those two approaches relates to processes and mechanisms and to whether they vary across species and situations. Johnston's interests and goals run almost orthogonal to this whole controversy. In his words, "Note that any such general principle [of adaptations of learning] is quite neutral with respect to the existence of general processes of learning.

If one is interested in understanding the processes and mechanisms of learning, regardless of whether one believes that learning involves a single set of mechanisms or many sets of mechanisms, one is apt to experiment on the roles of reinforcement, attention, interference, delays between stimuli, and so forth. That is, it is no accident that the "biological boundaries" investigators borrowed many experimental designs from the "general process" people - many of those designs are amply suited to elucidate processes and mechanisms. If and when those interested in an ecological approach to learning investigate the processes of a particular kind of learning, it would not be surprising if their experiments also resembled the traditional paradigms. What Johnston seems to be proposing is not new answers to the standard questions about the processes of learning, or new experimental approaches to those questions, but a new set of questions. The new questions will focus on what each animal learns and on how this learning is integrated into overall behavior. Questions of this type would not necessarily replace the old questions; however, there would still be a place for investigations of mechanisms and processes, and for the controversies that have occurred concerning general processes versus biological boundaries of learning.

Answers to Johnston's questions will not come easily; the "study of the general" can emerge only from a great many studies of the particular, especially when we are talking about ecological principles. Progress will require a persistent, systematic approach, but the payoff could be a genuine understanding of the evolution of behavior.

\section{Ecology and learning}

\section{Alan C. Kamil}

Departments of Psychology and Zoology, University of Massachusetts, Amherst. Mass. 01003

I believe that Johnston is correct in arguing that we must take a more ecological, adaptive approach to the study of learning. In this commentary I will supplement Johnston's paper by discussing recent theoretical and empirical developments in the ecological investigation of foraging behavior and their relation to the psychological study of learning. There are three points I want to make.

The first point is that there is no necessary conflict between psychological and ecological hypotheses about behavior. Consider a flycatcher sitting on a perch watching insects fly by. Suppose there are several types of insects that vary in dietary value and ease of capture. Which insects will the bird attempt to capture and which will it ignore? This question could be answered in terms of psychological mechanisms such as instrumental conditioning, discrimination learning, or attention and memory. It could also be answered in an evolutionary framework, for example by attempting to discover which available strategy is most adaptive. Assume that each theoretical approach was developed to the point where each yielded accurate predictions. The predictions of the two approaches would have to be similar, since each attempts to predict the same behavior. Thus the two approaches should be complementary, and we should try to take advantage of this complementarity. The research strat- 
egy Johnston suggests - find out what animals learn under natural conditions and then analyze how they learn it - will emphasize this congruence.

The second point I would like to make is that the development of task descriptions is well under way in the area of foraging behavior, and these task descriptions suggest many problems of considerable psychological interest for anyone concerned with learning. Let us return to the flycatcher and his diet selection problem. Ecologists have attacked these kinds of questions by developing mathematical or graphical models of foraging problems that are then solved for their optimal solution, usually the solution that maximizes rate of intake while foraging (see Krebs 1978; Pyke, Pulliam \& Charnov 1977, for reviews). MacArthur and Pianka (1966) developed one of the original models for diet selection. Time spent foraging in the case of the flycatcher can be divided into two components: search time (time spent sitting on the perch) and pursuit time (time spent chasing, capturing and consuming prey). Let us assume that the prey types can be ranked in terms of harvest value gained per unit of pursuit time. Then the optimal solution to this problem can be seen as follows: start with the highest ranking prey type as the sole item in the diet and keep adding additional prey types one at a time. The addition of each prey type will reduce search time per unit of food, because, as more prey are included in the diet, less time will be spent waiting for an acceptable prey to come by. But each addition will also increase pursuit time per unit of food, because the prey are ranked in terms of pursuit time. If the flycatcher adds prey until the decrement in search time equals the increment in pursuit time, the bird will maximize its rate of intake while foraging.

This analysis of the diet selection problem suggests that the animal needs a considerable amount of information to behave efficiently. Does the animal have such information, and, if so, how is it obtained? The model assumes that the animal does have the information, or at least that the animal acts as if it does; this generates some interesting predictions. For example, the MacArthur and Pianka (1966) model predicts that whether a relatively low-ranking prey item is taken or not should depend on the relative availability of higher-ranking items; it should be independent of the availability of the low-ranking prey. This prediction has been confirmed both in the field (Goss-Custard 1980) and in laboratory simulations (Lea 1979; Krebs, Erichsen, Webber \& Charnov 1977). In the case of diet selection, task description is well under way, and the results are raising significant questions whose answers will involve learning.

The optimal foraging approach is also being used to study other foraging problems (Krebs 1978; Pyke, Pulliam \& Charnov 1977). In addition to the optimal foraging literature, there have also been a large number of field studies on foraging behavior in the last 10 to 15 years. Many of these studies report on the foraging behavior of individual animals over time, which is exactly the data needed to construct task descriptions for naturally occurring instances of learning (Curio 1976; Kamil \& Sargent 1980). This literature makes it clear that, despite occasional claims to the contrary (Lockard 1971), learning can be important under natural conditions.

This brings me to my third and final point: given that psychologists have long been interested in animal learning, and given that what animals learn in the field is important, it should prove beneficial for animal learning psychologists to become more cognizant of, and involved in, the study of learning in the field. Three benefits seem most likely. First, as Johnston points out, many of the phenomena of the traditional animal learning literature may be artifactual, with little or no significance outside of the laboratory. Taking an ecological approach will allow empirical determination of the extent of this problem. Second, there are probably many learning phenomena that are most apparent only under natural condi. tions (e.g., Kamil, Peters \& Lindstrom in press). Taking a more naturalistic approach will help resolve this problem, which will increase the range of learning phenomena under study. Third, the behavioral mechanisms suggested by many field studies of foraging behavior are complex. If animal learning psychologists would bring their expertise to bear on these problems they could contribute significantly to improving our understanding of the natural world around us and the manner in which animals have adapted to it.

\section{ACKNOWLEDGMENTS}

The development of the ideas expressed in this paper was facilitated by discussions with many people, including Fred Lindstrom, Jerri. lynn Peters, Sonja Yoerg, and Herb Roitblat, and by research grants from NSF.

\section{The ecological approach to learning}

\section{John Kruse and Edward Reed}

Center for Research in Human Learning, University of Minnesota, Minneapolis - I , Minn. 55455

Johnston's paper is a very useful and provocative contribution to the discussion of the role of learning theory and research on learning within contemporary psychology. The optimism of proponents of general process learning theory has proved unfounded. Although phenomena such as autoshaping and learned taste aversion have expanded the domain of inquiry in learning theory, there are nevertheless a number of important evolutionary and experiential factors that do not seem amenable to traditional associative analyses. The realm of learning theory has shrunk. Whereas previously it encompassed virtually all of psychology, now it covers, at most, cautious generalizations beyond the laboratory by means of highly specific models of normal or pathological situations. Modern ethologists and "biological boundaries" theorists have, quite properly, emphasized the importance of speciesspecific aspects of behavior; but in so doing, they have never been brave enough even to attempt significant generalizations across species of ecological niches. Johnston is to be lauded for his attempt to transcend the limitations of previous approaches.

The ecological approach to learning advocated by Johnston differs from all previous approaches (general process or biological constraint alike) in two fundamentally important ways. Because Johnston is not as explicit about these differences as we would have liked, we shall attempt to heighten these contrasts in this commentary.

First, the ecological approach to learning differs from traditional approaches in how it motivates experimental research. Traditional learning theorists derived their empirical questions and methods logically from the several theories of stimulus-response (S-R), stimulus-stimulus (S-S) and response-stimulus (R-S) associations. (Ultimately, modern learning theory derives from British empiricism.) Under the influence of Pavlov and Thorndike, learning research became a systematic inquiry into how elements such as Ss and Rs become connected by virtue of neural plasticity as a result of experience. The assumption was widespread, if tacit, that these connectionist experiments could reveal the general processes of learning. The biological boundaries theorists deny this last assumption, but they still conduct their research within the connectionist framework. In contrast, Johnston urges learning theorists to derive their theories ecologically, not logically. While he is willing to assume that general principles may evolve out of ecologically focused studies, he is not willing to assume that general transspecies processes exist.

$$
\text { 111-11.7;11411, generalize from }
$$


studies done in disregard of relevant species ecology. Johnston,s position makes sense, for we are more likely to prove that general learning processes exist by testing hypotheses derived ecologically from a variety of species than by assuming that some such processes must exist just because laws of physical causality are species-transcendent.

The ecological approach to learning differs from other approaches in a second, perhaps more profound, way. Traditional psychological theories have been theories about animals and their capacities. In direct opposition to all of these earlier theories, ecological psychology attempts to formulate theories about animal-environment interrelations. Ecological psychology is as much a science of what there is to be behaved toward as it is a science of behavior. The fundamental concept of ecological psychology is that of the affordances of the environment for the behavior of animals (Gibson 1977; 1979; Reed \& Jones 1979; Shaw \& Turvey in press). Affordances are the things that exist in the environment toward which behavior can be directed: they are what the environment offers each animal for its behavior. The affordances of an animal's environment are the psychological characteristics of its ecological niche (its fundamental niche, not its realized niche, cf. Hutchinson 1978).

Johnston does not do justice to the radical nature of ecological psychology and affordance theory. If affordance theory becomes accepted, the entire edifice of associationism and connectionism will crumble. Affordances are not stimuli, nor do they cause stimuli or responses in organisms. Organisms are not sensitive to affordances, nor can there be thresholds for perceiving or acting on affordances. Affordances do not reinforce behaviors. Affordances cannot be associated, for they cannot get into an organism (as they are relations between an organism and its environment). To perceive an affordance is to perceive a meaningful aspect of the environment, but these meanings are neither innately given nor acquired via experience. With experience, an animal can become more acute at perceiving affordances and more adept at using them, but this improvement is not based on mechanisms of association, it is the result of the differentiation of organized skills of attention and action.

Affordances can be perceived, if there is information for them, by means of an active perceptual system attuned to pick up that information (Gibson 1966; 1979). Once perceived, an affordance can be acted upon (but it need not be) and, if the attempt is successful, the affordance may be realized. A long stick affords raking an object outside a cage into the cage, as Köhler's (1925) ape Sultan discovered. But not all manipulations of sticks are successful rakings. Af fordance theory emphasizes that behaviors are evolved achievements of an individual in an environment. From an ecological point of view, behavior is not touched off by stimuli, potentiated by drives, or reinforced after certain responses; behavior is, rather, an attempt to realize an affordance, an attempt that may succeed or fail. By and large, learning theorists no longer believe that there are laws that explain how Ss are connected to Rs; whether laws will be developed that explain how affordances are realized is very much an open question. The significant contribution that Johnston's paper makes is to raise this question.

\section{General process theory, ecology, and animal-human continuity: A cognitive perspective}

Janet L. Lachman and Roy Lachman

Department of Psychology, University of Houston, Houston, Texas 77004

Few explanatory systems in natural science have the power and generality of Darwinian natural selection; indeed, in the science of living systems, natural selection has no equal. One might, therefore, expect evolution and ecology to be among the most central themes guiding contemporary behavioral research. But they are not. Despite ritual espousals, no major psychological system has incorporated Darwinian theory into an effective, programmatic behavioral research enterprise.

Johnston would place the realities of adaptive specialization at the conceptual center of a behavioral theory and research program. We believe that eventually his position, or a similarly motivated one, will prevail. This is not to say that we agree with every detail of his criticism of existing approaches to the study of animal behavior, for we do not; but more on that later.

Our field is cognitive psychology, where the relevance of animal learning to human higher mental processes has been largely denied for two decades (Lachman, Lachman \& Butterfield 1979). It has been claimed that human higher mental processes and animal behavior are discontinuous that entirely different analogies and models are needed to explain the learning of animals and the cognition of humans. In the case of humans, one astute analyst has argued in this journal (Pylyshyn 1978) that there are no differences between systems of artificial intelligence and cognitive psychology. In our view, Pylyshyn's claim absolutely ignores two profoundly significant facts about human cognition: first, that it exists in an organism with a history of adaptive evolution, and second, that cognition developed in the service of the organism, s biological needs, not its intellectual ones. Human minds may play chess and do calculus, but it was their contribution to eating, mating, and escaping that gave such minds their staying power.

As advocates traditionally do, Johnston has set forth alternative positions in sharply competitive terms. On the one hand there is the ecologically motivated position he espouses, and on the other there is the traditional approach: "general process theory" and its derivative, the "biological boundaries" approach. Johnston's evaluation of the prospects of general-process theory is hopelessly pessimistic; this pessimism is based largely on the grounds that the commitment of general process theory to the existence of general learning processes has fostered ecologically arbitrary research methods that yield no insight into what animals actually learn in their natural environments. Admittedly, general process theory, along with other relatively mature paradigms in psychology and various disciplines, has generated its share of mindless parametric variations on research designs justified largely by expedience. However, general process theory is not philosophically and intellectually irreconcilable with an evolutionary orientation. "Ecologically arbitrary" research can play a valuable role in defining a set of capacities that operate in a given animal's natural environment or in any other environment in which it happens to find itself.

The "general capacities approach" can be viewed as an effort to discover those capacities of an animal that operate in all possible worlds. The question, "What can a rat (or dog, or human) learn in whatever environment it happens to find itself?" is a legitimate question that is not independent of natural history, since significant evolutionary development stemmed from abrupt, sometimes catastrophic, environmental alterations. Johnston,s question, "What does a rat (or dog, or human) actually learn in the single context of its expected environment?" is also legitimate; and, as Johnston argues, it has been badly neglected. But that neglect imbues the question with exclusive legitimacy.

Capacities, in fact, are not handled well in Johnston's approach, although they have preoccupied cognitive psychologists for two decades and must certainly play a significant role in any theory of learning. An animal learns to respond adaptively to its environment only within the constraints set 
by its capacity to perceive, conceptualize, and remember. Thus, capacities are analytically separable from either of Johnston's two queries: "What does the animal learn to do?" and "How does it learn to do it?" It appears that Johnston has merged the question of capacities with the question of what the animal learns, and thereby obscured a valuable analytical distinction. To illustrate, Johnston gives examples of research on "what behavioral problems an animal must solve" by citing Emlen,s $(1972 ; 1975)$ work on the indigo bunting, which orients itself by referring to configurational information distributed over groups of stars. Discovery of these birds' orienting responses required the prior discovery that indigo buntings are capable of perceiving, organizing, and forming representations of patterns that consist of points of light on a dark field, and that of all the things they might respond to when trying to solve orientation problems, these patterns somehow recommend themselves. These are capacities, but it is not clear whether they are part of Johnston,s "what" question or part of his "how" question. It would be analytically sharper, in our view, to devise such ecologically derived learning questions as "How does the animal learn to use the capacities it has to solve the problems it faces?" or, put another way, "How does the animal learn to do what it can to get what it needs?" There are three subquestions: (1) What behavioral problems must the animal solve? (2) What capacities does it bring to the solution of these problems? and (3) How does it match its capacities to the problem at hand? The general process approach may be well-suited to answering the questions of the second type.

Despite these criticisms, we are fully sympathetic with Johnston's call for an ecologically motivated approach to learning. In our own area of human cognitive psychology, we have recommended "evolutionary postulates" as a major heuristic in constraining models of cognition (Lachman \& Lachman 1979). However, there are unique difficulties in applying an ecological approach in the area of human higher mental processes. First, the adaptation par excellence of human intellect is its profound generality of purpose - its ability to solve a range of behavioral problems far greater than that of any other biological species. This adaptation makes it uncommonly difficult to answer the question "What behavioral problems must the (human) animal solve?" Second, unlike other species, humans have deliberately (and "artificially") altered their own environment. This alteration makes it inordinately difficult to distinguish "ecologically valid" from "ecologically arbitrary" research settings in the case of human beings. To these difficulties must be added the fact that the target behaviors of major social significance often involve behavioral problem-solving in the most artificial contexts human ingenuity has been able to invent (e.g., schools, submarines, spacecraft, courtrooms, inner cities, and the United Nations, to name only a few).

These difficulties do not mean that taking the ecological approach is impossible in cognitive psychology, for without it we will never understand human learning. The fact is that humans solve their vast array of ecologically arbitrary behavioral problems with mental machinery adapted to a prehuman environmental niche. No explanatory system can succeed if it does not deal with this stubborn, undeniable reality. On the other hand, ecological theorists who work primarily with nonhuman species should not underestimate the difficulties faced by an ecological approach to human learning. It may be impossible to bring ecological considerations to bear on the study of human cognition as directly as possible in the study of animal learning. Progress may depend on creative interplay between those cognitive theorists who incorporate evolutionary ideas and those who do not. Such interplay might begin with evolutionary interpretations of robust phenomena in human cognition. For example, one such phenomenon in human memory studies is the finding that sentences are more memorable if they are easily imaged. This effect was discovered in human memory research, which is similar to the animal research generated by general process theory in that neither has ecological or evolutionary motivation. Better recall is of ten attributed to better initial comprehension (i.e., imageable sentences are easy to recall because they are easy to understand). But why should imageability facilitate comprehension? In artificial intelligence or general process theory terms, there is no apparent reason. But an answer can be proposed in terms of human phylogeny. Human vision is essentially primate vision - it is threedimensional and acute. Such a specialized visual system probably developed as an adaptation to arboreal life, but it also facilitated the perception and memorial representation of objects. Because vision was acute enough to support it, fine distal discrimination among objects could emerge, and an equally refined conceptual ability could develop. In this view, the objects labeled by later primates (probably late Australopithecines\} pithecines) were visually-derived mental representations. If language developed in this way (i.e., parasitically on the visual system) it would not be surprising to find that the most facile applications of language are those that are most visualizable (i.e., imageable). In our laboratory, we have failed to eliminate visual processing effects in our studies of picture naming - or even to isolate them from semantic and lexical effects (Lachman \& Lachman 1980). Despite heroic efforts, we have not been successful, which suggests that linguistic and conceptual representations may be tied more closely to the visual system than to other modalities. [See also Parker $\&$ Gibson: "A Developmental Model of the Evolution of Language and Intelligence in Early Hominids" BBS 2(3) 1979.]

Explanatory efforts such as the one just described suggest new directions for human cognitive research. But perhaps equally significant is the potential for a new relationship between theories of human and animal learning. The relationship would require that one neither naively assume nor reflexively deny continuity of process in human and nonhuman learning, but rather that one use the natural history of the human species, coupled with an ecological approach, to suggest specific and investigable continuities. Such an approach would capitalize on current efforts in the study of both human and animal behavior, and at the same time support the needed development of an ecological focus in both areas.

\section{Species differences and principles of learning: Informed generality}

\author{
A. W. Logue \\ Department of Psychology, State University of New York at Stony Brook \\ Stony Brook, N. Y. 11794
}

Johnston,s article is quite correct in criticizing the anima learning researchers for lacking specific strategies for dealing with species differences in learning. Yet this lack is not it general due to these researchers' ignorance of species differ ences (contrary to what Johnston and many others - e.g. Seligman \& Hager 1972 - have claimed). Instead, it is base on a choice made by most past and present animal learning researchers to study only phenomena that they believe to be general and not species- or task-specific.

For example, in the early twentieth century, when the experimental study of animal learning was just beginning $k$ take shape, E. L. Thorndike (1965) - known primarily for hi description of The Law of Effect, which formed the frame. 
work for the study of reinforcement in animal learning - also emphasized the effect of species differences on learning: - All [species] are systems of connections subject to change by the law of exercise and effect. The differences are: first, in the concrete particular connections, in what stimulates the animal to respond, what responses it makes, which stimulus connects with which response, and second, in the degree of ability to learn - in the amount of influence of a given degree of satisfyingness or annoyingness upon the connection that produced it" (pp. 280-281). J. B. Watson, the founder of behaviorism, was so convinced of extensive species differences that he stated that it is impossible to generalize from animals to humans (Logue 1978). Skinner's (1959) work also has evidenced an acute awareness of species differences. He stated that "Difficult problems of idiosyncrasy or individuality will always arise as products of biological and cultural processes, but it is the very business of the experimental analysis of behavior to devise techniques which reduce their effects except when they are explicitly under investigation" (p. 375). In more recent times there have been numerous attempts to incorporate species differences into models of classical and operant conditioning and motivation (e.g. de Villiers 1977; Rescorla 1980).

Despite the clear recognition of species differences, all of the animal learning researchers are repeatedly accused of constructing general principles of learnig where no generality exists. This is because animal learning researchers who have chosen to focus on the study of general principles of learning have tended to give the impression that they are aware only of general principles (Logue in press a; in press b). In truth, animal learning has at times been hindered by an adherence to general principles where there is little generality. Yet the major challenges to such incorrect allegiances have come from within the animal learning community itself, such as Brown and Jenkins' (1968) study of autoshaping and Bolles' (1970) study of avoidance learning. In fact, some of the researchers who Johnston states have gone too far in abandoning general principles were themselves originally members of the field of animal learning (e.g., Shettleworth).

The history of the study of illness-induced food (taste) aversion learning provides an excellent illustration of how the animal learning community has and has not adequately handled species differences. Garcia encountered resistance when he first tried to publish his findings that rats more easily avoid tastes when the tastes have been paired with illness than when the tastes have been paired with shock (Revusky 1977; Seligman \& Hager 1972, p. 8). Later findings showing that such predispositions differed between species (Wilcoxon, Dragoin \& Kral 1971) also met with disbelief and attempts to dismiss the research as a result of inadequate controls (e.g., Bitterman 1976). Many members of the animal learning community had, in fact, focused on general principles of learning for so long that they could not believe that such differences could exist. These researchers had forgotten - or they had never been aware of - the reason for animal learning's original commitment to the study of general principles: that general principles are one aspect of learning whose study can be quite useful, since information thus obtained will apply to different species and situations (Logue 1979).

At present the existence of species differences in taste aversion learning is almost universally accepted in animal learning. Yet taste aversion learning is still pointed to as an example of the inadequacies of the animal learning approach. Johnston would avoid such embarrassments to the study of learning in the future by first observing animals in environments as much like their natural environments as possible in order to see what behaviors are adaptive for those particular environments. Unfortunately, the incidence of taste aversion learning in the wild is small. Taste aversion learning is a rare, but essential part of an animal's existence, so it is doubtful whether it would ever have been discovered through natural observation alone. This is one area in which the animal learning psychologists, working in an "artificial" laboratory, have had a great deal to contribute.

The ethologists recognize this contribution and do not criticize animal learning's description of the principles by which taste aversion learning takes place, although these findings were virtually all obtained in what the ethologists consider to be an unnatural environment. Apparently this is because these principles include species- and task-specific differences. Yet it is illogical to accept laboratory findings when they show such differences, and then to dismiss other laboratory findings of regularities, attributing the latter to the regularities of the laboratory setting. Recent taste aversion learning findings have shown, contrary to what was once believed, that although birds can easily form illness-induced aversions to visual stimuli (Logue 1980; and Wilcoxon, Dragoin \& Kral 1971), tastes may be the easiest stimuli to associate with illness for all species (Lett 1980; Logue in press c). Will the ethologists now reverse their position and denounce the principles of taste aversion learning, discovered by animal learning researchers, as the result of contrived laboratory situations?

In evaluating data from learning experiments, it is essential that a researcher have a firm grip on the ways in which species- and task-specific principles may arise and on the ways in which general principles may arise. Johnston lists three important ways in which general principles of learning may come to exist, but there is a fourth way. There are problems that all species face under many different situations. Because these problems have optimal strategies for solution, it is reasonable to expect that the same strategies would occur across different species and tasks. Johnston is concerned with how particular strategies are acquired during development; animal learning theorists are concerned with studying the strategies once they have been acquired. Examples of strategies include how an adult animal reacts to delayed reinforcement or to a choice between two intermittently available food sources. Different species can, and do, demonstrate similar behaviors in such situations, and such similarities are entirely consistent with evolution.

Yes, animal learning has at times neglected species differences, and yes, animal learning at present contains no consistent theory about how to incorporate species differences into learning principles. Such criticisms have inundated the literature for the past 10 years. But general principles of learning do exist and, as objects of study, they are as valid as speciesand task-specific principles of learning. Why not recognize the contributions of both the animal learning and the ethological approaches? Both are necessary for the study of the whole organism.

\section{A fourth approach to the study of learning: Are "processes" really necessary?}

John C. Malone, Jr.

Psychology Department, University of Tennessee, Knoxville, Tenn. 37916

Johnston describes three approaches to the study of animal learning, each of which is unsatisfactory, and all of which, taken together, do not exhaust the available approaches. The first view, general process learning theory (GPLT), posits a set of laws that are unconditionally general and thus apply at all times and under all conditions to all organisms. I have argued that such "laws" are no more than caricatures of the classical and instrumental learning paradigms and derivative princi- 
pies, and are always cast in the terms of a simple associationism (Malone 1973; 1975; 1978). The general paradigms have been successful in commercial animal training and human behavior modification but have not been spectacularly useful in advancing our understanding of learning in general.

The origin of the GPLT view is usually attributed to Pavlov, Thorndike, and Skinner by critics such as Seligman (1970). Actually, GPLT is a caricature of the message of these so-called exemplars; it is probable that few proponents or critics of GPLT have carefully read Pavlov $(1927$; 1932) or Skinner $(1931 ; 1966)$. Otherwise, the origins of GPLT would be more fairly placed with the distillations of textbook writers and derivative theorists.

The biological boundaries group is indeed cut of the same cloth and, in the stressing of "exceptions" to the "laws" of GPLT, almost gives the laws themselves an added legitimacy. Johnston points out beautifully the subservient relation of this approach to GPLT, and no further comment is required. I might only point out that the recent popularity of the biological boundaries position has led to an extremely frustrating state of affairs for those of us who are interested in learning but who have never given credence to GPLT, because our criticism of the biological boundaries view is taken by our colleagues in psychology and in zoology to be an implied acceptance of GPLT!

There must be alternatives to these approaches, but Johnston's "ecological view" is not the best, because it incorporates some of the same preconceptions that are imposed on the two approaches above. What is needed is the discovery of more empirical generalizations (principles) that describe orderly relationships between aspects of the environment and behavior. Pavlovian conditioning and the law of effect are two such empirical generalizations, and both have already been shown to be immensely useful in dealing with a wide range of important phenomena in animal and human learning. Their value lies in the range of situations to which they apply, and there is nothing to be gained in attempting to discover the real processes that underlie them. They are not in need of explanation because they are explanations.

However, if these and other principles are expressed in skeletal form, and if they are viewed as unconditionally general "laws," then we surely find that there are "exceptions." It is sad but true, as Johnston seems to realize to some extent, that we must take into account our species, its past experiences, its current environmental conditions, and our specific ways of defining environmental and behavioral events.

A concern with underlying processes, which Johnston's approach exemplifies, is very harmful at this stage of our knowledge. In sketching his ecological view, Johnston espouses a fairly specific theory of behavior that includes some very questionable assumptions. Despite his early argument against the supremely vacuous term "adaptation," he assumes that it is indispensable; that is, he believes that all animal behavior must be interpreted with reference to a set of "goals" in its environment. There is "natural" behavior and there are "natural" environments, and (natural) organisms are problem solvers that solve "adaptive problems." Our observations of natural organisms therefore take the form of the information processer's "task description." From these admittedly grossly biased ethograms we must glean the "what" of behavior, which henceforth is guaranteed to be limited to instances of problem solving. Subsequent work, whether in the laboratory or elsewhere, will seek the "how," the underlying processes/mechanisms.

Johnston's final summary of views proposes a general framework for guiding the long-term research of a large group of like-minded people who are concerned with developing a comprehensive theory of principles and processes for organisms in general. The ecological group studies behavior that is classified according to observer-defined notions of "tasks"; traditional comparative people study learning within taxonomic groups; and the third group studies populations that are oddly defined in terms of habitats or salient aspects of lifestyle.

These groups do not appear to be anything new; Johnston,s ecological group in particular appears simply to involve the compiling of task descriptions (the consciously biased ethograms) and the study of principles of adaptation that would require years to describe the simplest behavior. An alternative overlooked by Johnston suggests that we ignore processes, if processes are to be construed as the "secrets behind the phenomena." Instead, we should study any organism available under any conditions, including the most arbitrary or "unnatural," with no guiding theory other than the belief that there is order in behavior and that careful observation will reveal it. One gross regularities are found in one situation and in one species, we will have clues to guide us in our attempt to find order in the behavior of other species or in the same species under different conditions and in different situations. For us, "natural environment" simply means that setting in which we wish to be able to make predictions, whether it be a laboratory, a school, or a peat bog. We assume that orgainisms interact with their environments, and "adaptation" is acceptable in that sense. But adaptation, like "reinforcement," has acquired so many meanings and implicit theories (such as Johnston's) that both terms are best abandoned. As Kantor (1971) has pointed out, we need to know more about what organisms do, and we need more empirical generalizations that describe whatever order may be found in their behavior. If we insist that animals are "associating," or "solving problems," or doing the latter by means of the former, we will not advance our understanding far.

\section{Learning theory: Behavioral artifacts or general principles?}

\section{John A. Nevin}

Department of Psychology, University of New Hampshire, Durham, N.H. 03824

One of Johnston's major arguments is that the study of behavior in arbitrary, artificial laboratory settings may tell us nothing at all about regularities of behavioral organization and modification in the natural environment. I want to pose a complementary argument: that research in highly constrained, arbitrary environments may lead to principles that can guide analysis and interpretation of behavior in the wild.

From an ecological point of view, nothing could be more arbitrary than the study of simple operant behavior, main tained by schedules of food reinforcement or shock avoidance, and repeated during brief daily sessions until stable performance is achieved. Nevertheless, some principles of considerable generality have emerged from this line of research. For example, behavior is sensitive to the long-term statistical correlation between its rate of occurrence and the rate of food reinforcement (Baum 1973a), or to the proportion of food reinforcers that are dependent on response (Rachlin and Baum 1972; Lattal 1974). In a related fashion, avoidance behavior is sensitive to the relative reduction in shock rate that it achieves (Herrnstein \& Hineline 1966) or, stated otherwise, avoidance behavior is sensitive to the statistical correlation between response rate and shock rate (Gibbon, Berryman \& Thompson 1974). When food or shock termina• tion is arranged to occur at different rates for two different responses, the ratio of the two response rates or of the times 
allocated to the two responses roughly matches the ratio of food or shock termination rates (Herrnstein 1970; Baum 1973b; for a review, see deVilliers 1977). Finally, responses that are reinforced are frequently more resistant to changes induced by a variety of operations than are responses that are less often reinforced (Nevin 1974; 1979).

Because these findings have been replicated systematically in several species, using diverse apparatus arrangements, stimuli, and response classes, it is unlikely that the findings are artifacts of a particular experimental method. However, I agree with Johnston's distinction between principles and processes. The matching relation, for example, is quite general across species and situations, but it may result from somewhat different processes in different cases. Diversity of process does not weaken the value of the principle, and indeed may strengthen its generality. General principles of the sort summarized above suggest several observations:

1.The relations between behavior and environment may be studied effectively at a molar level, which involves extended time periods and many repeated episodes of behavior and stimulation. Rates, rate ratios, and correlations are necessarily estimated over large samples, and individual episodes become significant only when they contribute to the samples. The behavior-environment relation may then be conceptualized in terms of feedback at a molar level where behavior over extended time periods alters the environment and is in turn affected by it (Baum 1973a).

2. It is very unlikely that molar principles of the sort summarized above would be suggested by naturalistic observation designed to define task descriptions. The reason is that sensitivity to event rates, rate ratios, and correlations can be demonstrated only when the events recur reasonably often under stable conditions. When these conditions change from time to time, associated changes in behavior can be observed. Behavioral observation in natural environments, and the subsequent experimental analysis that such observation may suggest, is more likely to concentrate on rapid changes in behavior in discrete episodes (see Bolles 1970 on acquisition of avoidance), than are effects that require only one or a few trials for their expression (see Revusky \& Garcia 1970 on taste aversion), or sequences of events that occur only once in an individual,s lifetime (Marler, 1970, on acquisition of bird song). Thus, research in artificial settings is necessary.

3. Because there is reasonable evidence of the generality of principles developed in laboratory settings, the molar relations between behavior and environment cannot be ignored. These relations reflect properties of the behavior of real organisms and must be presumed to be adaptive in the natural world as well as in arbitrary environments. Properties of behavior that is isolated in the laboratory are no less relevant to behavior in the wild than are the properties of vision, worked out under the artificial constraints of the psychophysics laboratory, to the perception of the natural world. It is at least as valuable to consider naturally occurring behavior in the light of principles that are worked out in artificial situations as it is to take naturalistic descriptions as primary and then engage in an experimental analysis.

In conclusion, the study of behavior is a two-way street. Observation of behavior that is of obvious significance in the natural environment affects the interpretation of findings in arbitrary environments, and conversely, generalizations from the laboratory affect what we see as significant in the natural environment. Ideas about evolutionary fitness, ecological factors in behavior such as foraging and mating, and principles of behavior such as those summarized above are joined in a recent book that brings together workers with biological, psychological, and economic orientations (Staddon 1980). Cross-disciplinary exchange of this sort is far more likely to achieve generality in the understanding of behavior than is a viewpoint that discounts the findings of research in artificial environments as mere artifacts.

\section{A functional view of learning}

\section{Lewis Petrinovich}

Department of Psychology, University of California, Riverside, Calif. 92521

Johnston has reviewed the arguments that have led investigators to question general process views of learning. These views, since the time of Thorndike, have dominated the classical position of learning psychologists (see Petrinovich 1973 for a brief review of this history). The use of a term such as "constraints" involves a tacit acceptance of a uniformity view that holds that there is a general associative mechanism that typifies learning processes, and that this general law is abridged, or amended, by special factors. This view has little more appeal than any empirical generalization such as Thorndike's weak version of the law of effect, and it has little heuristic value to deal with ecologically relevant situations. (The term "weak" is not used in a value-laden sense. It is used to indicate that there is no commitment regarding the nature of the underlying motivational processes.) Such low-level inductive approaches result in a catalogue of behavior in a variety of situations and experimental conditions and has not led to strong general explanatory principles. The problem with such weak empirical generalizations is that they offer only a descriptive system in the guise of explanatory mechanisms.

An examination of the changes in general process theories over the years supports the conclusion that most of the classic general processes have been abandoned: the concept of primary reinforcement as a necessary condition for learning has been abandoned; the idea of plasticity regarding the associability of conditioned and unconditioned stimuli has been abandoned; and even the principle of contiguity as the basic law of association adopted by learning theorists from John Locke and all of the British empiricists, through Pavlov, Thorndike, and the learning theorists of the 1940s and 1950s has been seriously questioned.

We have, then, a situation in which our general laws have very limited applicability. Yet the commitment to traditional epistemological beliefs continues to govern interpretations of the data field. The general process laws seem to apply to neutral sets of events (neutral in the sense that they have little meaning to the behavior of animals in their natural environment). Thus our general processes may be operative in neutral situations where a major task for the organism is to discover what the concealed game is (Argyris 1975); or in the classical conditioning of a neutral conditioned stimulus to an arbitrarily selected unconditioned stimulus (one that is arbitrary in the sense that it has been chosen because it has an unprepared response (Seligman 1970). Thus we have a set of general laws of learning that might hold for certain classes of adult human behavior (perhaps that is why we cherish them) and that can be applied to the understanding of the acquisition of artificially created symbol systems: learning vocabulary, memorizing scientific postulates, remembering telephone numbers, converting currency, and other cherished human occupations.

In short, we have an artificial and anthropocentric system of laws. When we consider the behavior of organisms in their natural environment we find that the special "constraints" accountfor more of the variance than do the general laws. We should seek the molar functional principles that involve the teleonomic directions toward which behavior moves as a result of evolutionary principles (Petrinovich 1976). These principles consider behavior to be goal-directed through the operation of a program that is the result of natural selection. 


\section{Johnston: Learning theory}

"Each particular program is the result of natural selection adjusted by the selective value of the achieved end point" (Mayr 1974).

This view leads to a research program similar to that advocated by the ethologists. Such questions as what must an animal do to survive and reproduce, to what stimuli must it be responsive, what is the range and nature of the ecological conditions to which it must adapt, what are the other organisms with which it must interact, what is the nature of social systems, and what are the relevant social signals must be asked. This approach produces an understanding of what an animal can learn at certain times and in certain situations, and of how and where it responds to certain kinds of stimuli. Such an understanding will be difficult to obtain if the organism is viewed as an amended or constrained associative medium.

There is an important contrast between - as Johnston has phrased it - general process theory and general principle theory. This is not a trivial difference since the two theories lead to quite different methodological strategies which, in turn, produce radically different sciences.

General process theories tend to be organism-centered, to seek molecular-reductionistic explanations, and to rely as much as possible on systematic laboratory experimentation. General principle theory, on the other hand, tends to place equal weight on organismic and situational variables, to develop molar-functional explanatory principles, and to rely on nonmanipulative and representative research strategies as much as possible. The essential differences forced by a preference for systematic as opposed to representative designs have been discussed at length elsewhere (Petrinovich 1979; in press) and will not be repeated here.

Abandoning the organismically centered approach with its attendant molecular-reductionistic bias will result in a more satisfactory functional science of learning. This science will be characterized by the recognition that both the environment and the organism's input and output systems are probabilistic in nature (Brunswik 1952). The focus should be, as Tinbergen (1951) has argued, not on such questions as what can an animal learn, but rather on what an animal actually does learn under natural conditions. This emphasis moves the focus from hypothetical models of the mind to an analysis of the environment that the animal's behavior has been shaped to meet. Perception, learning, and memory, like evolution, are surely a matter of discovering what the environment is really like and adapting to it.

Functionally oriented research programs will allow us to understand the behavior of organisms in relation to environmental demands, to identify the potential avenues of stimulus-response mediation, and to move toward an understanding of the internal processes that mediate in the adjustment of the organism. Theories of behavior must be developed within the context of the environment because it is the environment that provides both the stimulation and the response supports for behavior.

The major problem in the development of behavioral theory has not been with the controlled experimental testing of alternative theories: psychologists excel at, and delight in, the design and analysis of complex experiments. The problem has been to discover the variables that should be included in our attempts to understand behavior. The very power of the experiment to magnify the effectiveness of a variable through control of other variables renders it almost useless for establishing functionally accurate probability estimates regarding the effect-size of variables.

These comments are not only germane for those who are interested in theories of learning. If one accepts the fact that the behavioral systems of organisms have been developed, through the agency of natural selection, to cope with the demands of the environment, it is equally likely that the nervous system is subject to the same organizational principles. If one is to understand the function of the neural systems involved in learning, then one would be well advised to consider such systems within the contexts to which they have been adapted.

\section{Is an ecological approach radical enough?}

\section{H. C. Plotkin' and F. J. Odling-Smee}

'Department of Psychology, University College, London WC FE 6BT, and Departments of Applied Biology and Psychology, Brunel University. Uxbridge UB8 $3 \mathrm{PH}$, Middlesex, England

We have great sympathy with Johnston and agree with almost everything that he says regarding the limitations and errors of general process and biological boundary theory. We also have little quarrel with the general program of observation and experimentation that he proposes. Our principal reservation is that the theoretical framework that he offers is not sufficiently different from general process theory in one very important respect. Johnston appears (see below) to view learning mostly in isolation from other forms of adaptation. As a consequence, workers who follow an ecological view may do things that are rather different from what they do now, but in their isolationist thinking they will not be conceptually far from the currently prevailing general process theorists. What is required is an even more radical departure. We have argued (Plotkin \& Odling-Smee 1979) that learning can be fully understood only if it is seen within the context of all the other ways that animals have of gaining and storing information about their world. This would include, at a minimum, changes in gene frequencies in the gene pool of the population to which they belong, variable development, and indeed, sociocultural information gain and transmission, if it occurs. We have also suggested a scheme as to the relationship of these different forms of information gain and storage and why learning ever evolves at all. Such a larger theoretical framework encourages a radically different way of thinking about learning, and it is this larger framewor that we miss in Johnston's target article.

We use the phrase "mostly views learning in isolation" because sometimes Johnston does not. He seems to posit some kind of association between learning and development, but in doing so he blurs the distinction between the two. We recognize that this criticism is premature, since Johnston is currently working on this very point, but nonetheless, in the present paper, the result is a conceptual tangle that is well illustrated by his phrase "ontogenetic adaptation. - Where does development end and learning begin? In this regard we would like to raise three specific points:

1. Not all development is variable or plastic. Mayr (1974) captured the distinction between invariable and variable development using the notion of open and closed gene programs.

2. There are many instances of variable development (open gene programs) that cannot possibly be considered to be learning. Plants, for example, show a very high degree of developmental plasticity. Even the plastic development of the mammalian nervous system as seen for instance, in the variable development of columns of cells in the visual cortex of kittens as a function of early visual experience, is frequently not attributable to learning if we use the term "learning" in its accepted sense.

3. The converse, that all learning is development, runs into certain difficulties. In the case of learning, the site of storage is always the nervous system. Variable development, on the other hand, is not necessarily organ-specific (this distinction is discussed at some length by Plotkin [1981]). Furthermore, development is characterized by a high degree of irreversibil- 
Johnston: Learning theory

ity, whereas learning is characterized by a high degree of reversibility. It is possible, of course, for prior development to be compensated for by subsequent development, but compensation is not the same thing as reversibility. Similarly, although no learner can ever return fully to some original, prelearning state, thereby indicating that the reversibility of learning is limited, we would still argue that the reversibility of learning makes it qualitatively different from development.

The other major criticism that we have of Johnston's article concerns the position adopted regarding a possible distinction between the logical and ecological structures of a task. First, in asserting that animals need only learn about ecology, Johnston is guilty of the error that he accuses others of committing; that is, he is assuming a truth rather than stating an hypothesis. Second, if he were to push his own ecological approach far enough, then we think that his distinction between the logical and the ecological would in any case fall away. Surely an alternative and testable position is that some properties of the environment are so basic (the arrow of time, for instance) that they are common to all known environments of all known learners. If this is true, then we should expect the processes of learning in all learners to reflect these common properties somewhere in their actual learning, as a function of convergent evolution. There should, therefore, be some universals of learning. In any given instance, of course, these are likely to lie well hidden behind a plethora of relatively superficial animal-specific, task-specific, and local environment-specific variables. However, they might be expected to show up in the abstract logical structure of the task itself. If this is true, then Johnston's downgrading of the logic of learning must be premature. If Johnston broadens his ecological horizons sufficiently, we feel he should agree with us. One other disadvantage to his position on this issue is that it leads to the closing down of some potentially valuable lines of research. Prominent among these are investigations into the ways in which early learning facilitates later learning, since it is possible that learners can learn logic from their learning of ecology, and hence may learn future ecological tasks more easily.

Our other reservations are minor. It is probably a matter of emphasis (and there is clearly room in Johnston's position for it), but we missed Piaget's (1971) point that learning is an active enterprise. Learners not only change their worlds, but they are also themselves changed by the act of learning. In Johnston's terms, learning is a dynamic interaction by which animals not only learn about their ecology but also change that ecology and in turn are changed by it.

Finally, we are not told how the question "What does this animal learn to do" can be translated into an empirical program. Let us pose a question: Is it possible to observe an animal under normal, free-living conditions, and, without any preconceptions as to what learning does and how it is different from other forms of adaptation, to say what the animal learns? For us, the answer is no. It is impossible. To use Johnston's example, Emlen (e.g., 1975) had to have a definition of learning that set it off from other adaptive responses, even if unarticulated, before he could take birds into a planetarium and even begin to know what to look for. Merely talking about "behavioral problems" involved in migration obscures the distinction between solving a problem using learning and solving it using other adaptive processes. In other words, being able to recognize learning by observing an animal in its normal environment requires at least an implicit statement as to the rules or conditions concerning an animal,s interaction with its environment that describe and define the learning requirement and demarcate it from other forms of adaptation. The answer to this takes us back to our original point: the relationship between learning and all other forms of adaptation. [See also Plotkin \& Odlin-Smee: "A Multiple-
Level Model of Evolution and its Implications for Sociobiology" BBS 4(2) 1981.]

\section{Learning theory in its niche}

\section{Howard Rachlin}

Department of Psychology, State University of New York at Stony Brook, Stony Brook, N. Y. 11794

I wish Johnston the best of luck if and when he begins the task of trying to create a general theory of learning from the mass of casual observation, half-hearted experimentation, and theoretical guesswork that goes by the name of ethological research. It might be a good idea if he would take the trouble to learn the aims and techniques of general process theorists in their natural habitat, so to speak, before setting foot on this path. Otherwise, ignorant of the pitfalls of general process theory, he might well be compelled to fall into them all over again. If it is not easy to discover the aims and goals of animals in simple, "unnatural" environments, it must be still more difficult to discover their aims and goals in "nature," where aims and goals will certainly be disguised. A snake slithering across the jungle floor may be in its niche, and is, by hypothesis, surviving. But what are its aims? What are its goals? If drive reduction theory stumbled because it was forced to postulate exploratory, manipulatory, and curiosity drives, I wonder how far a general ecological theory will progress as it tries to decide what that snake is trying to do. Is it avoiding the elephant,s foot, looking for food, taking exercise, doing all three at once (a more likely but more complex scenario). Or perhaps it has some particular snakelike desires for which there is no word in the English language. This, I am afraid, is a jungle from which Johnston and those who follow him will never emerge.

The ambitious set of questions that the theory is supposed to move on to as soon as it disposes of the preliminary question, "What does the animal learn to do?" will never be asked, because the preliminary question will never be answered. The question will be especially difficult to answer if the intrepid ecologists, along with Johnston, make no distinction between learning and development. Because then it seems that what the animal learns to do is exactly what it does - and that gets us nowhere. It does not help to restrict the search for the snake,s goals to those that " . comprise whatever state of affairs must be brought about, typically recurrently, in order that the animal may survive and reproduce"; many species of animals survive and reproduce quite well in the laboratory. Are all the experiments of the general process theorists thereby relevant? If the snake on the jungle floor turns out to be just out for a slither, must we assume that it can learn nothing during that recreational time? And if it is claimed that recreation is also necessary for survival and reproduction, what can a snake do that is not then necessary? We are back again to the circularity (encountered previously in the several "liberalizations" of Hullian drive reduction theory) that has been dubbed the Harvard law of animal behavior: "Under the most precisely controlled conditions animals do as they damn please." It seems that animals, under "ecologically meaningful" conditions, (i.e., imprecisely controlled conditions) must be at least as willful.

It is particularly interesting that Johnston sees Small's Hampton-Court maze as ecologically meaningful (at least potentially), whereas the T-maze is held to be irrelevant. This, on the curious grounds that in the T-maze the rat can learn only "the logical structure of the task to the complete exclusion of its ecological structure." But, according to Johnston, animals cannot learn logic. Johnston does not seem to realize that the purpose of the T-maze is to exhibit the logical structure of the task to the experimenter, not to the rat. 
Although Johnston has doubts about rats learning logic, he is confident that " . they must learn about their ecology." Does he really think that what the rats learned in Small's maze is better described as "their ecology" than as a series of left and right turns? Even Small (1901), who described the rat's behavior in mentalistic terms ("disgust" was one such) must have been less optimistic than this about their chances (as students as opposed to subjects) in college biology courses. If Johnston thinks that the T-maze is the logical conclusion of the development of laboratory apparatus to study the behavior of the white rat, I wonder what he must think of the Skinner box - probably beneath contempt; certainly beneath notice, since neither Skinner nor his box is referred to in the article. This is understandable, because Skinner's general process theory obviously does not fit Johnston,s characterization.

Johnston says that general process theory "searches for the invariant properties of animals," whereas the ecological study of learning is "a search for invariant relationships between animals and their environments." Perhaps Hull's theory might fit this characterization (I do not think it does), but Skinner's certainly does not. All agree that interaction between an animal and its environment is crucial. The question comes down to this. Will invariant relationships between animals and their environments be found in relatively simple environments or in relatively complex environments? It is conceivable that study of more complex environments will be more fruitful, although given those invariant relationships that have so far been found (there are several; interested parties may write to me for some examples), my money is on the simple environments. But the issue is between simple and complex environments, not between natural and unnatural environments. Johnston says "Natural phenomena are those that exist independently of our own investigations of them." But this is either true of all phenomena or (if you are a Berkeleyan idealist) none. In this context, "natural environment," like "natural breakfast cereal" and "natural underarm deodorant," is a term appropriate to advertising, not science. [See also Rachlin: "Maximization Theory in Behavioral Psychology" BBS 4(3) 1981 (forthcoming).]

\section{Known general principles of learning cannot be ignored}

\section{Sam Revusky}

Department of Psychology, Memorial University of Newfoundland, St John's, Newfoundland A IB 5S7, Canada

Johnston proposes to study learning under natural conditions by setting up task descriptions and determining how animals acquire the necessary behaviors. These task descriptions would be more specific than the abstract paradigms used in traditional studies of learning. The use of such methods would probably help to counteract the tendency of traditional students of learning to ignore ecological factors and types of learning that do not fit into traditional paradigms. But they will not produce a satisfactory overall picture of learning because they ignore clear evidence that learning is a general biological process.

Johnston accepts the classical behavioristic assumption that learning is an acquisition of behaviors. He infers from it that the existence of a general learning process, or even general principles of learning, is problematical because "of the wide diversity of phenomena which are revealed when learning is studied from a more naturalistic standpoint." In fact, the biological underpinning for most of learning is at the level of the nervous system rather than at the level of specific behaviors. This is why a wide diversity of learning phenomena, at least in vertebrates, follows the same basic underlying rules Learning is largely prediction of the future based on the past The rules for accomplishing this, like the rules of human logic, are trans-situational, and the vertebrate nervous system is adapted to them. I made this argument in more detail in a paper (Revusky 1977) cited by Johnston. Underlying similarities in the learning of such different tasks as poison avoidance, shock avoidance, and T-maze discriminations show that they all depend on the same basic principles. These principles are not species-specific or situation-specific. Johnston's methodology would obscure them because they are not obvious under natural conditions. Had Pavlov followed Johnston's advice, he would not have supposed that principles observed mainly in the study of salivation in dogs apply to a great variety of animals in a great variety of tasks. There is also a related general process of how instinctive reactions become attached to neutral stimuli that Moore (1973) delineated in pigeons, Pavlov in dogs, and Sevenster (1973) in stickleback fish. I do not see how these known general principles of learning can possible become irrelevant under natural conditions.

Dependence of a biological function on a common biological substrate in a variety of animals has classically been allowed to define a common biological process. Learning is such a process both because it depends on the vertebrate nervous system, and because there are similarities underlying learning under a wide variety of conditions. It cannot be supposed that the nervous system completely restructures itself for each condition. The parade example of independent evolution is flying in bats and birds, but these depend on different organs and are different in detail. Independent evolution occurs when a function is necessary in some species but is useless and expensive to maintain in related species. Learning is different because it is important for all vertebrates, despite various unwarranted claims that certain vertebrates do not bother to learn under natural conditions.

In the study of biological processes, ecological considerations usually have a secondary role. Johnston himself has demonstrated this in his reference to Wall,s (1942) work on the vertebrate eye. The curvature of the lens must vary under different ecological conditions so that light will be focused on the retina so as to allow the general process of vertebrate vision to operate. Regardless of how many pages Wall devoted to specific adaptations, the general considerations are central to his book and make his analyses of lens curvature physiologically meaningful. Similarly, although specific adaptations of learning exist, and although their past neglect has been harmful, they cannot be properly understood without emphasizing the general process that they modify.

Johnston claims that the cues used in food aversion learning depend on the habits of the specific vertebrate species. But a closer analysis based on very recent work of Clarke, Westbrook, and Irwin (1979), Galef and Osborne (1978), Garcia and Rusiniak (1980), and Lett (1980) shows that there is a common primary process of selective association of tastes with sickness in nearly all vertebrates. There is also a secondary process, called potentiation, that allows ancillary cues like the odor and color of food to become associated with delayed sickness in the presence of a taste. There are, of course, such species-specific adaptations as the capacity of a few bird species to associate the appearance of food directly with delayed sickness. These reflect the habits and environments of different species but, like the different curvatures of different lenses, they are modifications of a basic biological process and can be properly understood only within that context.

I think Johnston can ignore all this because he adheres to the antiphysiological bias of those ethologists who seem like embittered veterans of the long civil war in biology between naturalists and physiologists. Only this can account for his 
claim that most "of the experimental designs in the poison avoidance literature are minor variants" on "biologically arbitrary" designs. The study of how food is avoided when it produces poisoning necessarily involves manipulation of properties of the food and properties of the poisoning. Calling the food or its stimulus properties a conditional stimulus (CS) and the poisoning an unconditional stimulus (US) sets a general task description and defines the real scientific problem of how the animal learns that the CS and US are associated. It does not exclude analysis of specific developmental and ecological factors, which have been emphasized by many who find this vocabulary useful. Johnston has offered no useful alternative but has simply referred to "allegiance to the associationistic tradition." Nor has he identified any important learning effects analogous to the stain and fixation artifacts that occur in studies of anatomy. The capacity of any animal to learn anything whatsoever, no matter how natural or unnatural the experimental situation is, is relevant to the study of learning.

A few important instances of learning, perhaps birdsong and navigation, may turn out to be independent of the general process. In such cases, of course, ecological studies need not take the general learning process into account, and Johnston,s approach may be the best. It may also be useful for dealing with invertebrate learning. Furthermore, it may well be desirable for some investigators to use Johnston's approach in situations where I believe it is inappropriate, since they are likely to uncover phenomena that people holding to the approach I have outlined would not notice. But still, Johnston criticizes too much and ignores too much.

\section{Explaining diversity and searching for general processes: Isn't there a middle ground?}

\section{Paul Rozin}

Department of Psychology, University of Pennsylvania, Philadelphia, Penn. 19104

I wish we weren't always pushed into choosing between partially correct alternatives. I'm afraid the middle ground, which is often correct, is just not as appealing or attentiongetting as more extreme views. Johnston's paper is an intelligent and literate statement of an ecology-diversity position, but it stakes out a position that is too extreme and promises more than it can deliver. However, this may be the only way to drive a basic biological point home to those learning psychologists who try to ignore adaptive ecologicalevolutionary perspectives or sharply limit their relevance to the study of learning (Rozin, 1977).

Johnston describes the "biological boundaries" positions with considerable sensitivity to the substantial differences among them, but he then proceeds to treat these positions as one. Yet the view put forth by Kalat and myself (Rozin \& Kalat 1971; 1972; further amplified in Rozin 1976a; 1977) is in most respects more similar to Johnston's view than it is to those of most of the other biological "boundarists." In particular, I don,t think that our position is subject to any of the criticisms except perhaps the third one that Johnston makes of the biological boundaries position(s).

I have tried elsewhere (Rozin 1977) to explain why the least biological explanations of "anomalies" in the learning literature (Seligman 1970) have been most influential in psychology. I agree with Johnston that "constraints" or "biological boundaries," the widely accepted nomenclature for this set of positions, is inappropriate. Kalat and I used the term adaptive specialization (a term that is not congenial to psychologists) in order to emphasize shaping by environmental characteristics. The constraints terminology focuses on the limitations of adaptations rather than on their unique qualities. It implies that specializations are carved out of a general process organism. A modest extension of the boundaries approach would have us describing birds as constrained to fly, which is a clumsy and unproductive formulation.

I agree (as should be clear from my writings over the last 10 years) that task descriptions (functional analyses) should ordinarily precede mechanistic analysis. Imagine how much easier a process explanation of football would be to understand if one had an idea of the purpose of the game. I have tried to develop such an approach to explaining the mechanisms that omnivores or food generalists bring to bear on the problem of identifying useful sources of nutrition and avoiding toxins (Rozin 1976b).

We (Rozin \& Kalat 1971) selected the same major example as Johnston - Gordon Walls's (1942) classic work on the adaptive radiations of the vertebrate eye - to make our point concerning the value of studying diversity and organismenvironment fit. But the vertebrate eye story illustrates what is wrong with Johnston's position if it is taken as an exclusive prescription for the direction of research in learning, for the most impressive thing about vertebrate eyes is their similarity: goldfish, sparrow, and human eyes are remarkably alike. In fact, a more or less "general process" approach to vision has been very successful in psychology.

The problem and virtue of the ecological approach is that it focuses on the differences among species and environments. This is an extremely important perspective that is presented well by Johnston, and it is a view that psychology continues to ignore. But we cannot forget that all animals live in a world governed by a common set of physical laws and that there is a great deal that all environments have in common: events move forward in time, preceding events may help to predict subsequent events, temporally contiguous events are more probable predictors and causes, spatial contiguity is normally a condition for causation, and many events occur in cycles. These and many other commonalities could reasonably lead to some common principles across motivational systems within the species, and across species. One might argue that universal aspects of the world would normally be built into organisms, leaving the less predictable matters to be dealt with by plastic mechanisms. However, in some cases the universals are relations (such as the temporal sequence of prediction), and experience must fill in the particulars (e.g., what predicts what, and what objects in a general spatial scheme hold what locations).

In terms of evolution, I am much more sympathetic to the strong position outlined by Johnston. It is hard to imagine how general process types of solutions could evolve de novo; it is much more likely that they arose as adaptive specializations (Rozin 1976a). However, given the frequency of common problems in the environment, the high incidence of many useful adaptations (e.g. homeostatic systems, inhibition), and the fact that types of solutions are constrained by the properties of the nervous system and the sequence of development, it is quite reasonable to assume that mechanisms that evolved to deal with local adaptations become, in some cases, more and more widely available to solve new problems in the course of evolution. This increased accessibility view of the assembly of more general systems from specific adaptations is the core of a theory of the evolution of intelligence that I have developed (Rozin 1976a).

Animals face the problem of developing useful representations of the world and effective plans of action to deal adaptively with the problems they face. I agree that functional or ecological analyses are an important step toward understanding and analyzing the role of experience in solving 
these problems. Johnston and I also agree that the traditional learning paradigms probably account for only a small percentage of the plastic mechanisms and adaptations evolved by animals, and that there is absolutely no reason to assume that processes of learning have not undergone an adaptive radiation. Johnston has laid out a sensible program to explore the diversity and organism-environment fit of these adaptations. But we should not limit ourselves to explaining the general principles of diversity. Recent psychological research has exposed an ability to learn about contingencies the learning of associations only insofar as the prior event predicts the occurrence of a biologically meaningful event (Rescorla 1967). This finding makes biological sense, and links with a universal aspect of the environment. (Note that thinking in terms of the shaping of learning mechanisms to fit the environment might have moved us to this position earlier, but nonetheless, this important advance did come out of nonbiologically oriented psychology). There is some evidence of surprising "general problem solving" abilities in some animals (Premack 1976), an adaptive ability that is again compatible with a general process view.

Therefore, while I feel that Johnston is suggesting valuable directions for research in learning, I think we should also be thinking of how universal environmental features are represented in animals, and how this might relate to general processes. I think it is reasonable to concentrate on either function or mechanism, on diversity or commonality. But it is not reasonable to forget that we are dealing with animals with an evolutionary history, who have to get around in real worlds and solve real problems. Johnston's position has value as a corrective for a strong and detrimental bias in the psychology of learning, but the universals in ecology hold out real hope for some general processes.

\section{Adaptive modification of behavior: Processing information from the environment.}

\section{Wolfgang M. Schleidt}

Department of Zoology, University of Maryland, College Park, Md. 20742

T. D. Johnston's view evokes in me the feeling of déjà vu. I agree with his view, find little to argue with, and nothing to attack. I do find, however, that several points in his paper blur under closer scrutiny, and $\mathbf{I}$ have chosen to discuss briefly three points that I consider important for the ongoing discussion.

First of all, Johnston never states what he means by learning. It remains unclear whether proponents of any of the three views (general process, biological boundaries, and ecological) can agree on one single definition of learning. Hovland (1951), for example, defines learning as "the change in performance associated with practice and not explicable on the basis of fatigue, or artifacts of measurement, or of receptor and effector changes." This view is useful for the connoisseur of human cognitive skills, whose orientation is toward the higher functions of the central nervous system (CNS) in man, but whose focus is much too narrow for both the ethologist and ecologist. The latter must deal with the adaptive actions and responses of a variety of organisms, independent of the supporting structure (the "hardware" or "software" that stores and processes information concerning the organism's environment).

Habituation to specific, recurring features of the environment is a very common form of adaptive process which, in "lower" animals without a clearly defined CNS, is likely to occur at the receptor level. In an ecological approach to learning, habituation, fatigue, and receptor and effector changes must be included in the definition of learning as potentially adaptive responses to certain environmental information. The key phenomenon to be dealt with is the individual organism, which "feeds" on information from its particular environment (as well as on energy) to maintain its stakes in the game of "inclusive fitness." We are not satisfied to know that information has entered the organism and changed its behavior. We must have proof that this information enhances inclusive fitness - that the change is "adaptive." "Adaptive modification" (Lorenz 1965) is a concept that seems to fit this requirement quite well. It encompasses those processes of behavioral change due to environmental information that were previously barred from the definition set forth by the general process proponents (habituation, sensory adaptation, etc.), and includes the bulk of the original "learning" (leaving open, for the moment, the question of whether or not most learned people have the highest inclusive fitness).

A second point, which is not spelled out clearly enough for my taste, is the question: What makes Johnston,s approach ecological"? Traditionally, ecology is especially concerned with interrelations between groups of organisms and their environment (Odum 1959). Those groups are usually populations of conspecifics and, as populations, they are characterized by statistical measures; the variables interacting with other statistical variables in a "typical" environment, are conceived of as an n-dimensional niche (Hutchinson 1957). When Johnston presents his "ecological approach to learning," however, he describes problems that concern the interaction between organism and environment on a one-to-one basis. The branch of biology that deals with this problem is ethology. I realize that, as a label for a new approach to a difficult problem, the word ecology sounds better to the ears of some scientists than does ethology or sociobiology. However, since we are dealing with an especially difficult problem, it is important to scrutinize its epistemological basis and to clarify the topography of the elements, especially the relationship between an organism and its environment (Schleidt \& Crawley 1980) and the levels of organization on which these interactions occur (Novikov 1945; Odum 1959; Schleidt 1981).

The nature of the environment is the third point I shall address. Johnston has outlined the problem very well and has emphasized the necessity for a careful description of the environment. I feel, however, that some problems must be solved very early in order to prevent conceptual blunders. "Environment" has a wide range of meaning, from the simple presence of a specific stimulus (light on the retina) to an all-encompassing animistic power. More specifically, the general process proponent will view the environment as a complex (sum?) of all stimuli associated with a response; the classical ethologist is likely to imagine the individual organism surrounded by its personal Uexkullian Umwelt bubble (Uexkull 1957); the ecologist, however, will see the environment as an abstract hypervolume to which the species adapts (Hutchinson 1957). For the ecological approach, as visualized by Johnston, the Stimulus-Response concept is too simplistic, and the "collective environment" of a group is not applicable in the individual case. The most promising course, in my view, is to refine the Umwelt bubble concept: The subject under consideration is surrounded by objects, each carrying potentially vital information, and each having the potential to influence the subject's energy balance. These objects can be food, conspecific competitors or potential mates, predators, tools, obstacles, or neutral items, bathed by the rays and gases of the atmosphere, and held to the ground by gravity. The subject interacts in this general setting with one or more objects, managing its time-budget according to a strategy that maximizes the chances for achieving high inclusive fitness. It 
Johnston: Learning theory

will take great epistemological skill and sophistication to devise a useful format with which to describe the environment where learning, as an adaptive process, takes place.

\section{The ecology of learning: The right answer to the wrong question}

Barry Schwartz

Department of Psychology, Swarthmore College, Swarthmore, Penn. 19081

Today, the idea that "everything important in psychology ... can be investigated in essence through the continued experimental and theoretical analysis of the determiners of rat behavior at a choice-point in a maze" (Tolman 1938, p. 34), must seem a grandiose vision built on massive ignorance. Indeed, the growing influence of ethological ideas on learning theory, together with the development of cognitive psychology as a viable experimental science, makes it appear as though studying rats in T-mazes is about the surest possible way to learn nothing at all about anything - except perhaps rats in T-mazes. And Johnston makes clear in his target article precisely why "general process" learning theory has turned out to be misguided. He argues convincingly that if one wants to understand how learning influences an animal,s adaptation to its environment, one must begin by understanding the challenges that a particular species' environment poses. The problem is that although behavioral ecology may be the way to answer questions about how animals solve problems of survival, those are not the questions that motivated learning theory. Thus Johnston's alternative may be the right answer to the wrong question.

To appreciate what learning theorists were after, one must connect learning theory to its history. Most major learning theorists, including Watson, Thorndike, and Skinner were seeking laws of human nature. They studied animals as a methodological convenience, a convenience justified by a commitment to empiricist epistemology, which largely determined what they thought an account of human nature would look like. The legacy of British associationism suggested that human nature was shaped by experience; that experience built simple elements into complex combinations via mechanisms of association; and that there were no significant pre-established categories of knowing that required that the learning theorist, in order to arrive at an understanding of human nature, study people directly, as they learn the things they normally learn. This constellation of ideas made quite plausible the belief that one could construct a picture of complex human activity by studying simple creatures in simple situations. The natural sciences had already taught us that this was the way to discover the key elements - the general principles.

As learning theory has developed, and as research issues and methods have grown increasingly complex, the relation between minor theoretical controversies and the experiments designed to resolve them on the one hand, and the big, motivating questions about human nature on the other, has grown increasingly obscure. Indeed, many modern learning researchers are interested in problems of animal learning for their own sake and participate in the field for that reason. For the current generation, Johnston's criticisms and suggested alternatives are apt and appropriate. But the sins of the children should not obscure the aims of their parents. Watson, Thorndike, and Skinner never lost sight of their goals. This is clear from their persistent efforts to apply principles of learning derived from the animal laboratory to complex, human settings. Indeed, the appeal of using successful human applications for validating laboratory principles continues to characterize the research of Skinner and many of his disciples.

If one appreciates that the aim of learning theory was to discover universal principles of human nature, one can understand some of the shortcomings of the demonstrations of . constraints on learning" identified by Johnston. In the last decade, a number of findings have indicated two things: first, that much of what any given species learns, it learns in specialized ways; and second, that phenomena peculiar to particular species creep into experimental environments that were thought to be purged of such phenomena. These two separate types of findings have led people to conclude that typical learning procedures will tell us nothing important about people - and very little about the animals we study. Johnston criticizes this literature as being one that takes pot shots at learning theory without offering an alternative. Much of the literature contains ad hoc argument, and all of it is parasitic upon the very ideas it criticizes. I think Johnston is right. Indeed, I have made similar arguments myself (Schwartz 1974). However, if one appreciates that the goal of learning theory was to render an account of human nature, the pot shots are enough. They make it clear that learning theory won't tell us much about human nature. There is no need for a programmatic alternative like Johnston's unless one is interested in animal behavior. Learning theory was not.

If the reformed learning theorist retains the goals of his past, but seeks new means to attain them, are Johnston's means the right ones? At present, there is certainly no reason to believe so. Johnston himself points out that the enthusiastic application of sociobiological ideas to human behavior is hardly more than an optimistic handwave at present, and perhaps even less than that (Lewontin 1979). And the alternative program outlined by Johnston seems to have limited applicability to the task of explaining human nature. First, consider the idea of a "task description." This implies that there are set tasks to be described. This may be true of some species, and it may be true of many aspects of human childhood (e.g., learning language, learning number concepts, learning to walk, etc.), but it hardly seems plausible once early childhood is passed.

What are the "tasks" faced by human adults? Which . natural" environments should we observe to derive our task descriptions? As a rule, people do not live in natural environments. They live in environments of their own construction. I think the general learning theorist knew this and intentionally constructed artificial environments precisely because flexibility was such an insistent characteristic of human behavior that it was important to avoid studying "natural," though perhaps parochial, aspects of human nature.

And what does it mean to say that a behavior is "natural"? Johnston suggests that "natural phenomena are those that exist independently of our investigations of them. " Even in the domain of nonhuman behavior, this idea is fraught with difficulty. As Johnston points out, "methodology interprets the world and it does so in terms of the theoretical predilections of its designers. "What he perhaps fails to appreciate is that this is just as true of simple observation as it is of anything else. What we see as natural phenomena will be at least partly determined by the theoretical apparatus that gets us to look. Phenomena do not come in interesting classes. What we look for will be influenced by what we think is important to find. And what we think is important to find will influence what we see in nature. In this sense, there are no phenomena of interest to science that exist independent of our investigations. For Skinner, it is a "natural" phenomenon that human behavior is governed by its consequences in lawful ways, just as it was for Adam Smith. But one can look at the same 
phenomena that Skinner looks at and conclude that the full-blown instrumentality of human behavior is the product of modern, industrial capitalism and not something "natural" at all (Polanyi 1944). Learning theory fails as a general theory of human nature - but not because it is unnatural. It follows, therefore, that attempts to replace learning theory with something that is closer to natural events will not necessarily solve learning theory's problems.

To summarize, I am confident that Johnston's alternative to learning theory will provide valuable answers to questions about the determinants of animal behavior. However, because learning theorists have different questions, a solution to their problems probably lies elsewhere.

\section{ACKNOWLEDGMENT}

Work for this paper was supported by NSF grant BNS 78-15461

\section{An ecological theory of learning: Good goal, poor strategy}

Sara J. Shettleworth

Department of Psychology, University of Toronto, Toronto, Ontario, Canada M5S 1 A1

"Biological boundaries" to learning have been threatening to become mere adjustments to parameter values, while theorists ignore their implicit message that learning is best understood as part of an animal's adaptation to its natural environment. Johnston is to be commended for his accurate analysis of the shortcomings of existing "biological boundaries theories" and for his attempt to develop a broader biological framework for the study of learning. But, as someone who is basically in agreement with his goals, I must disagree with his strategy.

However broadly it is defined, learning can be analyzed in terms of three aspects or levels. The most basic aspects are the learning paradigm, a description of the experience that is supposed to cause behavioral change in the situation of interest, and the learning phenomenon, or how behavior changes. Learning principles are then paradigmphenomenon relationships, that is, statements about how given types of experience affect behavior. This definition of the word principle agrees, therefore, with Johnston's. Learning theory traditionally goes beyond principles, however, to a concern with learning processes - the mechanisms (not necessarily physiological) that are responsible for observed paradigm-phenomenon relationships. For example, in the case of habituation, the paradigm is repeated exposure to a single stimulus; the phenomenon is the waning of some initial response to the stimulus. Thus the habituation process has been hypothesized to be either specific to the particular stimulus-response relationship or to constitute sensory or motor fatigue. Observation of the effect of a novel stimulus on the habituated response (a dishabituation experiment) differentiates between these two classes of processes.

It can readily be seen from this analysis that, as Johnston points out, natural selection can act only at the level of learning principles. It acts only indirectly on learning processes. Moreover, as the history of learning theory amply illustrates, a given paradigm-phenomenon relationship can often be produced, in principle, by any of several mechanisms. Therefore, a concern with how learning is adaptive that is, the function of particular behavioral outcomes from particular sorts of experience - is orthogonal to the traditional concern with mechanism. This does not mean the two sorts of concerns are independent (Shettleworth in press), but without further development a framework based on the adaptive function of learning has little place for the traditional concerns of learning psychologists.

Johnston calls for a descriptive catalogue of the ways in which experience affects behavior in the normal course of development on the assumption that something general will eventually fall out of the catalogue. The principles, he suggests, may be in terms of taxonomy or functional categories (feeding, sex, etc.). At the very least, the effects of experience will be classifiable as being maintaining, facilitative, or inductive. This is not a theory that deals with learning mechanisms, however, and I think the possibility is quite remote that anything general will emerge from it concerning the mechanisms by which experience affects behavior. The problem is, as D. S. Lehrman (1970, p. 28) put it, that "Nature selects for outcomes, not processes of development." In the case of bird song learning, for example, the adaptive outcome (or task description, in Johnston's terms) is uttering a reason • able approximation to the species-typical song in the right situations. The pathways by which this ability develops are varied indeed (Marler \& Mundinger 1971). Some species must hear their species-typical song at a certain period of development; others are stimulated to produce species-typical vocalizations through contact with juvenile conspecifics; others may sing effective songs with no experience of conspecific vocalizations at all. There still seems to be no comprehensive account of the functional correlates of this tremendous variation in the way song develops, and perhaps none is possible.

The problem is even more severe with developmental phenomena like those studied by Gottlieb (e.g. 1978), which Johnston considers important material for an ecological theory of learning. Such phenomena as the development of auditory preferences in ducklings are far from the traditional concerns of learning theory in those cases where there is a need for adjustment within the animal's own lifetime to events that are in principle unpredictable, except on the basis of individual experience, and that may vary within the animal,s life (cf. Plotkin \& Odling-Smee 1979). [See also Plotkin \& Odling-Smee: "A Multiple-Level Model of Evolution and its Implications for Sociobiology" BBS 4(2) 1981.] In addition, concern has usually been with how an animal,s experience of environmental events affects its behavior with respect to those events. In cases that have these characteristics, the requirements of adaptation should constrain quite narrowly the form that learning principles may take, thus indirectly determining learning-mechanisms. An ecological theory of learning can more profitably begin with a concern with experience of this sort rather than with the broad atheoretical description suggested by Johnston. Indeed, collecting analyses of development carries no guarantee that any but the most unspecific effects of experience will be found.

For learning that is narrowly constrained by the requirement to adjust to a priori unknown events, a detailed and productive ecological theory may be possible, and indeed, is already starting to develop (Hollis in press; McNamara 1 Houston 1980; Shettleworth in press). In order to optimize some parameter that is presumed to be related to reproductive success (such as rate of energy intake while foraging) animals must sometimes have information about some aspect of their individual environment such as the location and distribution of food. In such cases, we can predict what the properties of learning or memory in that situation should be. Possible mechanisms that could generate the optimum or near optimum behavior can be hypothesized and data can be collected to determine which behavior the animal is using. This is already going on in the analysis of foraging (Lea 1979; 
McNamara \& Houston 1980). As in Johnston's approach, this enterprise will lead in the short run to "local principles," but these will deal with the relationship between mechanism and adaptive function. It is interesting that McNamara and Houston,s (1980) investigation has already provided an example of how a learning mechanism that can produce an adaptive outcome in a natural situation is less than optimal in some laboratory partial reinforcement paradigms.

In summary, Johnston has made a valuable contribution to learning theory by carrying to their logical conclusion the implications of "biological boundaries" data. But in one respect he has gone too far: he expects learning theorists to broaden their concerns to phenomena that are too remote from those with which they have traditionally been concerned. In another respect, he has not gone far enough: his program is too unfocused and atheoretical. There already exist examples of how the analysis of adaptation in particular situations can make contact with the details of learning mechanisms. Further developments of this sort are more likely than Johnston's program to bring some ecological content into theories of learning.

\section{Missing variables in studies of animal learning}

\section{Wally Welker}

Department of Neurophysiology, The University of Wisconsin, Madison, Wisc. 53706

The aggregate behavioral-neurobiological sciences have revealed that "learning" phenomena consist of multiple processes, are anatomically distributed, and are polydeterminate. There are many kinds of learning. All levels of the nervous system play a role in learning. In fact, neural substrates of learning phenomena are not localized to any structure or circuit. Rather, they appear to be distributed among several neural systems. To properly understand phenomena of learning, six major codeterminants must be addressed: environmental stimulus contexts, nonneural anatomical characteristics, perceptual selectivity, cognitive competence, motivational pertinence, and the available behavioral repertoire. Johnston's review emphasizes the importance of environmental and behavioral factors, particularly those that occur in "natural" environments.

All learning is dependent on spatiotemporal features of the environment, and Johnston does well to emphasize that the study of animals in "natural" stimulus contexts is the most pertinent strategy, evolutionarily and developmentally, for analyzing adaptive learning. Ethologists and ecologists have known about and demonstrated this for over a century, but the most numerous, convincing, and articulate demonstrations have been produced during the last 25 to 50 years. Johnston rightly advises learning theorists to acknowledge such cumulative wisdom.

All studies of learning have been forced to deal with, to describe and measure, specific behavioral sequences - the second half of the Stimulus-Response (S-R) equation. Here too, Johnston acts as spokesman for the many ethologically and ecologically oriented behaviorists who have demonstrated the importance of studying the alterations of specific "natural" behaviors in their "natural" situations.

Topics that are not dealt with (but surely implied) in Johnston,s discussion of the three contrasting approaches to a theory of learning are (1) an animal's specialized skeletal muscular and other nonneural anatomy, (2) its sensory- perceptual neural specializations, (3) its cognitive capabilities, and (4) its unique motivational dispositions. A large body of experimental, analytic, and descriptive research attests that an essential contribution to learning phenomena has been made by these several neurobiological substrates. Because this is true, such features, which are "natural" features of each animal's makeup, are as important as "natural environments" and "species-typical" behaviors in assessing an animal's learning capabilities. It certainly must be clear by now that the spatiotemporal organization of the circuits and systems of the brain comprise the biological substrates to which any biologically adequate theory of learning must be addressed. This is particularly important because each animal's "natural" adaptive behavioral transactions, and the modification of behavior by learning, are directly determined by the several "natural" specializationsof its brain anatomy and physiology.

I do not see how purely environmental and behavioral theories of learning can proceed far if they do not concurrently examine neurobiological phenomena in depth. "Natural" environments and "natural" behaviors constitute only two of the essential ingredients that must be examined, understood, and utilized in searches for understanding of the growth, modification, and development of an animal's adaptive transactions with its environment.

There are several other issues to which theories of learning (general and special) must attend. First of all, there are several varieties of learning phenomena, that are operationally distinguishable from one another and that are referred to by such terms as "conditioning," "imprinting," "association," .. adaptation," "familiarization," "incubation," "fixation," etc. These abstractions only touch the surface of the problem. To be credible, general theories of learning must consider these several aspects.

There is also a problem regarding the dichotomous use of the terms "natural" and "artificial" in referring to either environments or behaviors. Although it is important to study learning in environments within which an animal group has evolved, I think the case is somewhat overstated when it is explicitly assumed that any response an animal makes under the constraints of laboratory environments is not "natural." Indeed, it seems well demonstrated that all animals are able to respond to some stimulus energies and patterns that they rarely encounter in nature, which indicates only that they are often constitutionally prepared to deal adaptively with novel niches, or at least with niches different from those in which they may now exist. I think that the more appropriate emphasis for an ecological approach to learning would be one in which "task descriptions" are made for several environments that are parametrically varied so as to include a wide variety of possible situations, including some laboratory conditions which may otherwise be characterized as "artificial." If such an approach were followed, it is possible that an animal's specific learnings would be found to be tailored to environments that differed from those in which it was originally found. Such a possibility cannot be excluded a priori. It is certainly known that many animals inhabit widely changing niches or habitats - some complex and diverse, some simple and constrained - such as occur in normal diurnal, seasonal and annual cycles. The question is: What and how do animals learn in these different situations?

These few comments are to be viewed as addenda to, not critiques of, Johnston's suggestions for promoting more environmentally relevant studies of learning. His critiques of alternate approaches, and his ecological and species-typical emphases are valuable and constructive. His fine review should provide another corrective contribution to a shortsighted controversy of long standing, for the issue is still not fully resolved. 


\section{Author's Response}

\section{An ecological approach to a theory of learning}

Timothy D. Johnston

North Carolina Division of Mental Health, Research Branch, Dorothea Dix Hospital, Raleigh, N.C. 27611

Of the various kinds of criticism one's work may elicit, there are two that I find most valuable: The first kind comes from those who agree enthusiastically with one's effort and, in that spirit, offer suggestions for its improvement. The second kind comes from those who feel that the whole enterprise is thoroughly misguided, and so force one to defend the assumptions on which it is based. Having elicited both kinds of criticism (as well as some other kinds), I hope that I can use this response to gratefully acknowledge my supporters and, perhaps, to encourage my detractors to reconsider their objections. Some highly sympathetic commentators have received less attention in my response than they deserve. The commentaries of Charlesworth, Kamil, Kruse \& Reed, Lachman \& Lachman, Petrinovich, and Welker, in particular, raise a number of important points that amplify and extend my arguments - points with which I am in substantial agreement. For obvious reasons, I have concentrated instead on providing rebuttals to those commentators who have been especially critical. In addition, I have organized my response in the way that I have found most helpful as a reader of previous issues of this journal: in terms of topics, cross-referenced with the names of commentators (see Table 1). This will, I hope, make it easy for both readers and commentators to evaluate the strength of my rebuttals.

1. Generality and theories of learning. Several commentators argued that the very existence of general principles propounded within the general process tradition is an effective argument against my position. Logue suggests that one may focus either on general principles of learning, or on species typicalities; she contends that psychologists have simply chosen to focus on the former rather than the latter. However, it seems logically muddled to claim that principles exist as objects of study in the same way that, say, the phenomena of food selection do. General principles are constructed, not studied directly, which is demonstrated by the fact that they can be declared wrong or inapplicable, and then discarded. The point at issue is whether the general principles constructed by learning theorists are applicable to the natural phenomena of learning and, if not, whether we should retain them.

Revusky's argument is similar in spirit to Logue's: He claims that the general principles successfully constructed from studies of learning under artificial conditions must also be important for learning under natural conditions. I cannot accept the logic of this argument. It is surely an empirical matter whether or not the conditions under which such principles hold in the laboratory also occur in nature; for the great

majority of laboratory paradigms, this matter still remains to be investigated. If such conditions did not occur, then clearly the general principles that were based on them would indeed be irrelevant in nature. Nor do I claim that general learning principles are necessarily irrelevant in nature (cf. the last sentence of Nevin's commentary), for that would simply be

Table 1. (Johnston). Issues discussed in this response and the commentators who raised them

Issue Commentator(s)

1. Generality and theories of learning

2. Functions and mechanisms of learning

3. Adaptation and the evolution of learning

4. The concept of naturalness

5. Experimental design and the ecological approach

6. What is a task description?

7. The ethological tradition

8. What are psychologists really interested in?

9. Describing the environment

10. Learning and development
Bateson; Ghiselin; Logue; Nevin; Plotkin \& Odling. Smee; Revusky; Rozin

Bateson; Garcia; Gonzalez \& Yarczower; Hinde; Kalat; Kamil; Nevin; Revusky; Shettleworth Alley \& Shaw; Barlow \& Glickman; Garcia; Ghiselin; Hailman; Kamil; Lachman \& Lachman; Logue; Malone; Nevin; Plotkin \& Odling-Smee; Revusky; Shettleworth; Welker

Alley \& Shaw; Kruse \& Reed; Lachman \& Lachman; Rachlin; Revusky; Schleidt; Schwartz

Gonzalez \& Yarczower; Nevin; Rachlin

Bateson; Barlow \& Glickman; Gonzalez \& Yarczower; Hailman; Hinde; Lachman \& Lachman; Malone; Plotkin \& Odling-Smee; Rachlin; Revusky; Rozin; Schwartz

Barlow \& Glickman; Gonzalez \& Yarczower; Charlesworth; Hailman: Hinde; Schleidt

Garcia; Hinde; Kalat; Revusky; Schwartz; Shettleworth

Alley \& Shaw; Charlesworth; Kruse \& Reed; Malone; Petrinovich; Plotkin \& Odling-Smee; Rozin; Schleidt

Kruse \& Reed; Plotkin \& Odling-Smee; Rachlin; Schleidt 
making Revusky's error in reverse. However, so long as those studying learning in the laboratory show no inclination to find out what conditions obtain in the natural world, we will never know one way or the other.

There are a number of good ecological reasons to suppose that there exist general principles that are applicable to learning under natural conditions. The existence of phyletic constraints on the evolution of learning (Ghiselin) is one, and the probable similarity of adaptive problems faced by many species is another. Plotkin \& Odling-Smee and Rozin make the latter point cogently, and I see no reason to doubt that they are correct in principle. However, it will be important to determine which of the adaptive problems faced by different species exhibit the relevant similarities, and that issue cannot be decided by reflection [See also Plotkin \& Odling-Smee: "A Multiple-Level Model of Evolution and its Implications for Sociobiology" BBS 4(2) 19811

Revusky claims that logical rules for predicting the future are transsituational and that, insofar as learning involves predicting the future (a metaphor with which I am not entirely happy), principles of learning must be transsituational too. That argument takes too much for granted. The rules of logic are one way of predicting the future, and they have been developed by logicians precisely because they are transsituational. But that is no reason to suppose that a set of rules that successfully predicts the future for a particular animal in its particular environment must either look like the rules of logic or be transsituational beyond the limited range of conditions that the animal must face. What is important for the animal is that it be able to learn in the particular environment it does inhabit, not in all environments it might inhabit. The fact that animals can learn the rules of logic to some degree is certainly interesting, but it does not demonstrate that those rules are of any significance to the animal in its natural environment. That bears can learn to ride bicycles does not mean bicycle riding plays any role in the animal's relationship with its natural environment; it is only, in Dennett,s (1978, p. 569) elegant phrase, "a surprising fact of elusive theoretical significance. - This does not mean that it has no theoretical significance, but rather that whatever significance it may have is not apparent and requires demonstration. [See also Cohen: "Can Human Irrationality Be Experimentally Demonstrated?" BBS 4(3) 1981.]

It may well be, as Logue and Revusky suggest, that the ecological methodology I propose would obscure the general principles of learning discovered in the laboratory. That would surely be a fault of the principles, not of the method; general principles are not important only because they are general. As Bateson rightly remarks, they must also be relevant. If the ecological approach obscures the prominence of existing general principles of learning, it is because such principles are of relatively limited relevance in nature.

I do not mean to imply that the ecological approach has no faults; I am sure that it does, but obscuring existing general principles of learning does not count as one of them. Nevin also seems to feel that the fact that the traditional approach has produced general princi- ples constitutes a point in its favor that I have overlooked. I question neither the existence of those principles, nor their applicability to the psychological laboratory; what I question is their relevance to the world outside that laboratory (see also topic 4 , below).

2. The functions and mechanisms of learning. The question of the relation between functional and mechanistic explanations of learning pervades much of the current debate in the field and was raised in a number of ways by several commentators. Although the ecological approach emphasizes functional explanations - partly by redressing the imbalance that characterizes much of the study of learning - no adequate theory of learning can be either purely functional or purely mechanistic.

Several commentators (Bateson, Hinde, Kalat, and Shettleworth) claim that an ecological theory of learning will be purely functional, and they criticize me for implying that an understanding of mechanisms will follow from an approach based on functional considerations. It is certainly true, as Bateson, Hinde, and Shettleworth remark, that knowing the functional context in which a particular kind of learning normally occurs does not yet make it possible to predict its mechanisms. I suspect that the opportunism of natural selection means that this kind of prediction will always be rather imprecise, but I see no compelling reason to rule out the possibility that certain kinds of adaptive problems will turn out to be associated with particular kinds of learning mechanisms. Surely such correlations are precisely the kinds that have been explored so successfully by behavioral ecologists studying foraging behavior, mating systems, and social communication, providing the basis for our evolutionary understanding of these phenomena. It turns out that certain kinds of foraging behavior (mechanism) are best adapted for the consumption of certain kinds of food (function), although the fit is certainly imprecise. I hypothesize that there will turn out to be equivalent relationships between learning mechanisms and the functions that they serve. The precision of those relationships will have important implications for our understanding of the evolutionary lability of learning, that is, the extent to which phyletic constraints limit the adaptive tailoring of learning mechanisms (see topic 3, below).

Both Kalat and Shettleworth suggest that functional concerns are orthogonal to mechanistic concerns, implying that there is an even weaker relationship between ecological principles of learning and the mechanisms by which learning occurs. Certainly it is possible to study either mechanisms or functions independently; indeed, nonfunctional investigations of mechanism characterize the tradition in psychology that I criticize. My argument is that functional considerations should be used to provide a focus for studies of mechanism, the focus being defined by task descriptions. Task descriptions identify functions for which we must then supply mechanistic explanations, using the experimental paradigms currently available when possible, and devising new paradigms when existing ones fail us. Thus, in my view, the ecological approach to learning involves a much closer relationship between 
functional and mechanistic explanations than Kalat and Shettleworth believe, but it is not necessarily a relationship in which functional explanations entail or predict mechanistic ones (cf. Bateson and Hinde). Kamil provides an excellent statement of the proper relationship in his commentary; I am in close agreement with the points that he makes in this regard.

But is it not possible, as Gonzalez \& Yarczower, Kalat, Nevin, Revusky, and Shettleworth suggest, to use functional principles to interpret the results of mechanistic studies executed in the absence of any ecological concerns? In principle it is, but I think a research strategy that puts functional considerations before, rather than after, the analysis of mechanisms is greatly to be preferred for at least two reasons: First, as past experience has shown, it will probably be difficult enough to understand learning without expending a lot of needless effort studying mechanistic phenomena that have no functional significance. Putting functional considerations first could help to eliminate a lot of work that might prove in the end to have been unnecessary. Second, by continuing to put the study of mechanisms first, our investigations are likely to be constrained by the experimental paradigms and theoretical questions already characteristic of a nonfunctional tradition. This would reduce the chance that important functional questions not readily apparent from the traditional perspective could ever be answered, or even posed, except by accident. I think that Revusky recognizes this at the end of his commentary when he acknowledges that the traditional approach may cause some kinds of learning to be overlooked. My concern is that, from a functional point of view, these kinds of learning may turn out to be among the most important.

Garcia would place the study of mechanisms, especially physiological mechanisms, at the center of research on learning. While I agree that understanding the physiological bases of learning is an important goal, I do not agree with Garcia that nonphysiological accounts of learning are necessarily circular. Explaining the fact that an animal avoids certain foods on the basis of prior (behavioral) encounters of a particular (perhaps species-typical) kind seems to me to provide a valuable extension to our understanding of learning.

Incidentally, the history of comparative psychology does not bear out Shettleworth's claim that functional issues are "remote" from the traditional concerns of psychology (see Hull 1929, 1937; Brunswik 1952; Petrinovich 1979). Like Kalat, I think that a return to the traditional functional orientation of comparative psychology, an orientation that was abandoned in the middle of the century, would be in order.

3. Adaptation and the evolution of learning. Any approach to the study of learning that takes functional considerations seriously will have to deal with problems of the evolution of learning and the question of adaptation. Like most investigators concerned with ecological and evolutionary problems, I consider the concept of adaptation to be central to any ecological understanding of learning (Johnston \& Turvey 1980). Thus I am surprised that Malone writes that I argue "against the supremely vacuous term 'adaptation'. - I did issue some cautions about its use, suggesting that the term was often abused in biology, but I did not imply that this diminished its importance if the concept was used correctly. Rigorous treatments of the concept of adaptation are available (e.g., Sommerhoff 1950), and I doubt that any biologist would seriously consider abandoning it altogether.

Ghiselin points out that organismic structure and function are not completely determined by adaptive processes, and I appreciate his valuable comments on phyletic constraints in the evolution of learning (see also Gould 1980). This is an important consideration (also raised tangentially by Barlow \& Glickman) that is all too easily overlooked when one approaches a problem with an ecological rather than an evolutionary emphasis. I also agree with the suggestions of Alley \& Shaw and Plotkin \& Odling -Smee that the overall adaptive and evolutionary context must be considered by the ecological approach, and with Welker's underscoring of the relation between learning and other aspects of the animal's biology.

Revusky raises the issue of phyletic constraints when he notes the evolutionary conservatism of the vertebrate nervous system. However, the relevance of such conservatism to the issue of general phylogenetic principles of learning must depend on the extent to which the nature of learning is determined by those aspects of brain function that remain unchanged in evolution. Our present understanding of the neural basis of vertebrate learning does not seem secure enough to support Revusky's strong assertions on this point. Although I am sure that some such general effect of neuroevolutionary constraints will be found to exist, I am reluctant to assume this as a basis for research into animal learning.

I also doubt that there is an adequate basis for Revusky's remarks about independent evolution and the usefulness of learning. Intuitive assessments of how "useful" a trait may be to a given animal are exceedingly misleading in discussions of evolution. The potential usefulness of a trait has nothing whatever to do with the probability that it will evolve; this probability depends instead on the selective benefits conferred by the trait and on the available genetic and phenotypic variability in the population (see Williams 1966, for a clear discussion of this issue). The phylogenetic generality of learning cannot be defended by claims of .. general usefulness." If Revusky wants to make the modified claim that "learning is always of selective benefit, - then he will have to gather evidence to support that claim, and I doubt that such evidence will be forthcoming (Johnston, in press).

These problems with the concept of adaptation also conflict with Hailman's suggestion that my remarks about adaptation are self-evident. They may well seem self-evident to an evolutionary biologist, but I doubt that they do to many students of learning. There is, for example, an unfortunate tendency to regard everything an animal does as necessarily adaptive, just because the animal does it. Nevin's remark that "properties of the behavior of real organisms ... must be presumed to be adaptive in the real world just as in arbitrary environments" shows that my concern on this point is not unfounded. In describing taste aversion learning as essential to the existence of all animals, 
Logue seems to believe that the adaptiveness of particular kinds of learning can be determined intuitively. This is the "adaptive story-telling" that has been so vigorously criticized by Lewontin (1979). The mere fact that we can tell a good universal adaptive story about taste aversion learning does not mean that it is in fact universally adaptive. Nor, I might add, does it mean that all animals necessarily learn taste aversions under natural conditions, regardless of what they do in the laboratory. The adaptiveness of a trait must be demonstrated, not assumed or assessed intuitively, and this is never an easy undertaking. Nevin's analogy with processes of vision is in fact a poorly chosen one in view of recent work by Breitmeyer (1980) on visual masking, which strongly suggests that at least some "properties of vision, worked out under the artificial constraints of the psychophysics laboratory," (quote from Nevin's commentary) are indeed irrelevant to the perception of the natural world.

Barlow \& Glickman suggest that the concept of adaptation would be made more useful if it were defined in more-or-less rather than all-or-none terms. I agree, but I do not think that the route they propose to take is the right one. Although the issue continues to be debated by evolutionary theorists, I think that there are compelling arguments against treating adaptation and fitness as synonymous terms (Dobzhansky 1956, 1968). Fitness is a measure of reproductive success; hence Barlow \& Glickman's strategy produces, for example, the odd result that a mule is not adapted to its environment because it has zero fitness.

Alley \& Shaw chide me for not respecting the mutuality of animal and environment in my discussion of adaptation, but I see the concept of adaptation as a way to unpack that mutuality, rather than simply as a statement of it (Johnston \& Turvey 1980, p. $155 \mathrm{ff}$ ). Indeed, Alley \& Shaw seem to place too much emphasis on adaptation and come perilously close to asserting that animals are necessarily adapted to their environments in every respect, despite cogent arguments to the contrary (Lewontin 1978; Gould \& Lewontin 1979). While I am certainly sympathetic with their thoroughgoing ecological attitude, I have difficulty translating it into a program for research on learning without first taking some intermediate steps that will unpack the intimate relationship that they espouse between the animal and its environment - a relationship about whose existence I agree. I am proposing that we attempt to begin that unpacking.

Two commentators raise a point that may be relevant to the issue of preadaptations (Bock 1959) - those traits that an animal already possesses and that later turn out to be adaptive under changed environmental conditions. Lachman \& Lachman and Welker suggest that an animal's performance in ecologically arbitrary learning tasks may say something about the species' ability to deal with a novel niche. This kind of performance calls to mind Kuo's (1967) concept of a behavioral neophenotype for which he made much the same claim. I would like to see further discussion of potential insights that this idea may produce.

Garcia suggests that studies of the physiology of learning should form the basis for our attempts to understand the evolution of learning. However, the
Functional properties of learning abilities, rather than their physiological basis, gives them their selective value; and I suspect that adaptive explanations of the physiology of learning will turn out to be virtually unattainable. Physiological studies may help to answer some questions about the evolution of learning, especially those that concern phyletic constraints on evolutionary change, but I doubt that the relation between physiology and function is sufficiently close to allow physiological study to produce major insights into the problem of adaptedness.

There is no doubt that it will be both conceptually and empirically difficult to study learning from the adaptive point of view. It may be, as Malone suggests, that even for simple behaviors working out principles of adaptation will take years, but I do not see this as a cogent criticism of the ecological approach. If it turns out to be difficult to understand learning, and I fully expect it will, so be it. We will just have to live with that fact and find ways to simplify the problems. Shettleworth suggests that we focus initially on those examples of learning in which there is a fairly direct relation between the experience on which learning is based and the skill being learned. This is a useful suggestion, and the literature on foraging behavior to which Shettleworth alludes, and which Kamil discusses in more detail, is an excellent example of such a strategy in action. More heuristic principles of this type are needed

4. The concept of naturalness. The terms "ecology" and "natural" have suffered from popular overexposure in recent years. Gould (1978, p. 119) remarked that "Common usage threatens to make 'ecology' a label for anything good that happens far from cities." Both terms have legitimate technical uses, however, and I think that Rachlin is too harsh when he dismisses the term "natural" as more appropriate to advertising than to science.

Arguments about the importance of the concept of naturalness often seem to reflect a fundamental disagreement about the aims of science; these arguments are therefore philosophical rather than technical. This is not the place for an extended philosophical discussion, so I shall briefly describe my own philosophical position (which is by no means an unusual one), and then evaluate the commentators' criticisms of "naturalness" from that point of view. Science begins as an attempt to explain such natural phenomena as the movement of the planets, the existence of diverse species, or the coordination of complex behavior. In some cases, we employ experimental techniques to elucidate the phenomena that interest us, and such experiments are most useful when they test hypotheses about those phenomena. If we hypothesize, for example, that the specific anatomical connections between nerves and muscles are responsible for the coordination of some behavior, then we may test that hypothesis by altering those connections and observing the behavioral results of our intervention. Surgical manipulation of the connections between motor nerves and limb muscles in amphibians produces characteristic behavioral abnormalities that are important in deciding between competing hypotheses about the development 
of locomotor coordination (see Jacobson 1970, Ch. 7, for a review of these experiments). But any neurobiologist who pursued the study of those abnormalities for its own sake would be rightly accused of wasting time and effort. He would have confused the phenomenon to be explained (normal locomotion) with a different phenomenon (the locomotor abnormalities), which was created to decide between competing hypotheses. The experimentally produced abnormalities are of no interest in themselves, but only insofar as they help explain normal locomotion; if they did not help explain normal locomotion, then they would be mere curiosities - "surprising facts of elusive theoretical significance" (Dennett 1978, p. 569). Although these experimental abnormalities share certain physical characteristics with normal locomotion (for example, they involve movements of the same limb segments), they occupy a logical position that is quite distinct, for logically, normal locomotion and locomotor abnormalities stand on opposite sides of the interface between scientific inquiry and the phenomena to which that inquiry is directed.

If the preceding example provides an accurate analysis of the experimental method in general, then the relevance of the concept of naturalness to the study of learning should be apparent. The learning in which animals engage when we are not investigating them ("natural learning") is what we have to explain. In pursuing an explanation, we may create certain developmental abnormalities ("artifacts"), such as chaffinches that sing a species-atypical song, or pigeons that peck at colored keys. These phenomena are of interest only insofar as they clarify the natural phenomena of learning. If this example seems less convincing than the locomotor example preceding it, I think it may be for two reasons: First, normal locomotion is much easier to observe and describe than is normal learning, so the artificial nature of the locomotor abnormalities is much easier to appreciate. Second, the kinds of manipulations that produce developmental abnormalities in studies of learning are much more benign than those involved in studies of locomotion. However, I maintain that these differences reflect differences in technique, not in logical form.

According to this argument, it appears that Revusky's claim that all instances of learning, under any conditions, are equally relevant to the understanding of learning, is too sweeping and imprecise. Some instances of learning are relevant because they are natural; others are relevant because they explicate natural learning, and others are simply not relevant at all. A decision, for any particular instance, as to which of these characterizations applies cannot be made without appealing to the concept of naturalness.

To call a stimulus, situation, or experience "natural" isto identify it as characteristic of the animal's natural environment, since what is natural for one animal may be unnatural for another. Attempts to define "natural environment" are, I freely admit, fraught with all sorts of theoretical and empirical difficulties, but of course that in no way reduces their importance. As a first step toward an adequate definition, I have suggested that we define the natural environment as that environment which the animal occupies by virtue of its phylogeny and species-typical ontogeny. There is in this definition an attempt to treat the environment as a dynamic entity with a temporal as well as a spatial structure (Mason \& Langenheim 1957). Environments change through evolutionary time as well as through ontogenetic time; as the individual grows and matures, its relationship to its surroundings changes (on the latter point, see Alley \& Shaw's commentary). The problem of incorporating dynamic considerations into the definition of "natural environment" is a very difficult one, for which I know of no solution at present. Further development of the excellent points raised by Alley \& Shaw, Kruse \& Reed, and Schleidt with regard to environmental description (see topic 9, below) will, I am sure, be part of any solution.

Defining the natural environment for some species will be much more difficult than for others; Homo sapiens is the most obvious problem case. Schwartz correctly raises this issue and Lachman \& Lachman (1979) have made a very interesting attempt to resolve it (see also Lachman \& Lachman's commentary).

5. Experimental design and the ecological approach.I am accused by Gonzalez \& Yarczower and Nevin of believing that research done under artificial laboratory conditions is necessarily irrelevant to ecological concerns. It is important to be clear on this point, so let me reiterate what I stated explicitly a number of times in my target article: The use of artificial experimental conditions is essential to the understanding of learning from an ecological point of view. The important point is that such conditions cannot be chosen arbitrarily. The choice of experimental conditions must be based on knowledge of the animal's natural environment. This has been accomplished, for example, in studies of migratory orientation (Emlen 1972) and bird song (Marler 1970). The particular experimental conditions chosen by Emlen and by Marler for studying how such skills are learned were designed to test hypotheses based on their subjects' respective natural environments; these conditions were artificial but not arbitrary. Often the best way to analyze a complex developmental process is to simplify the conditions under which it occurs, but the nature of the simplification involved is crucial. Simplicity per se will not lead to useful insights (contrary to Rachlin's thesis). The aim of experimental simplification must be to tease out relevant experiential contributions to development, not simply to permit ease of measurement.

Gonzalez \& Yarczower observe that some principles derived from nonecological experimental designs have turned out to be applicable to ecologically relevant forms of learning. This is true, but it is hardly a good argument in favor of pursuing a nonecological approach. What we would like to find is an approach that allows us to formulate just those principles of learning that explain the natural phenomena of interest, not an approach that produces such principles only sporadically. The ecological approach may not turn out to be the best way to accomplish this, but it is a more viable candidate than one that ignores the role of ecological principles in experimental design.

6. What is a task description? The idea of task descriptions for learning generated more disagreement and 
misunderstanding than I had anticipated. Some ethologists (Barlow \& Glickman; Hailman; Hinde) felt that I was trying to claim too much originality for this stage in the analysis. Insofar as task description involves describing behavior, of course I claim no originality whatever; however, I do think that the systematic application of the idea of task descriptions to the study of learning would be a novel and important direction for future research.

The task description defines what the animal does, so that we can go on to determine how it learns (if it learns at all) to do it. Barlow \& Glickman, and Plotkin \& Odling-Smee attribute more to the task description stage than I had intended. Description cannot determine what aspects of behavior depend on what kinds of learning; rather, it provides a detailed account of the behavioral skills possessed by the animal at different times in its life. To determine how experience contributes to the development of these skills, that is, to determine what the local principles of adaptation are, requires that we go beyond description to experimental analysis. I think that part of the confusion may arise because these commentators would like to distinguish learned from unlearned behavior, and they recognize that observation alone will not suffice. However, I question whether this is a useful strategy in any case (see footnote 3 in the target article).

Bateson correctly observes that one contribution of the task description stage is to set limits on parameter values for experimental analyses of learning. I would extend this role to cover those that are varied as well as those that are held constant. In a similar, albeit more general vein, this allows the investigator to define the developmental problem in the laboratory in the same terms as in the natural environment. The more precise that definition can be made, the more penetrating will be the resulting analysis of development.

I am puzzled by Malone's complaint that task descriptions are "consciously" and "grossly" biased. Certainly, as Schwartz points out, any description of a complex event is bound to be selective - if that is what Malone means by "biased." The aim of task description is to make clear what one is selecting and why, and ethologists have usually done very well in that regard. But Malone's implied claim that the paradigms used by learning psychologists are not selective is simply bizarre! How a Skinner box can provide a less selective account of an animal's behavior than does careful observation of its behavior in nature is quite beyond me. I am equally puzzled by Rachlin's apparent concern that I propose to uncover animals' beliefs, desires, and intentions in the task description stage. Surely we are past the point of worrying about teleological implications whenever goals are mentioned in discussions of behavior. Or is Rachlin still haunted by the ghost of mentalism?

Gonzalez \& Yarczower, and to a lesser extent Revus$\mathrm{ky}$, criticize me for focusing on the animal's behavior in formulating the question, "What is learned?" But that is surely an appropriate focus for an ecological approach, since it is behavior that determines whether or not the animal will cope successfully with its environment. No amount of cognitive processing or associating of stimuli and responses is going to ensure survival unless it gets translated into adaptive behavior, as Lachman \& Lachman point out. Learning skills exist today because they generally produce adaptively successful behavior in their possessors' natural environments; examining the nature of that behavior is therefore the most appropriate starting point for an ecological study of learning.

The role of the task description may perhaps be highlighted with reference to the example of the vertebrate eye, which I discussed in my target article, and which Revusky and Rozin both use as grounds for proclaiming the value of a general process approach (although Rozin proposes this as only a supplement to a more ecological approach). It is true that there are many important similarities in visual anatomy and physiology throughout the vertebrate phylum, and that a general process approach to vision has enjoyed great success in many respects. I suggest that this is in large part because the "task description" for vertebrate vision is also very similar across a wide range of species; the structuring of light by environments is, after all, very closely determined by universal laws of physics. Whether such close determination will turn out to obtain for those aspects of the environment that set the task descriptions for learning is a moot point.

7. The ethological tradition. If there were an ethological tradition in the study of learning, then I am sure that it would look very like the approach that $I$ have advocated. Many of the concerns reflected in my target article are, as Barlow \& Glickman, Charlesworth, Gonzalez \& Yarczower, Hailman, Hinde, and Schleidt point out, the same as those that have motivated ethological studies of behavior. However, ethologists have not produced the kind of broad, conceptually unified approach to the study of learning that has emerged in the ethological study of other kinds of behavior, such as mating systems, foraging strategies, social organization, and animal communication. In all those areas there is a broad consensus among ethologists on a range of important theoretical questions to be addressed, and there is abundant evidence of a concerted effort toward resolving those problems. That is not the case with regard to learning.

I do not believe, as Barlow \& Glickman think I do, that ethology is inherently limited in its ability to produce general insights into learning. Rather, I feel that because of the historical focus by ethologists on problems other than learning, ethological studies of learning have not, in fact, produced many such insights. If my article had been about behavior in general, rather than learning in particular, then my arguments would indeed do a major disservice to the ethological tradition; with regard to learning, I think they do not.

8. What are psychologists really interested in? The psychology of learning, Schwartz argues, is really directed toward understanding human behavior, not animal learning; he thus implies that the arguments in my target article are simply beside the point. While achieving an understanding of human behavior may well have been part of the historical impetus for the development of the field, and while it may also be a 
concern of some modern workers such as Skinner, I do not think that Schwartz's arguments carry much critical weight. My own arguments are not concerned with the question of the applicability or inapplicability of studies of nonhuman animals to the understanding of human behavior. They are concerned with the question of whether current approaches to the study of animal learning are the best way to advance our understanding of animal learning. Unlike Schwartz, I think that many psychologists regard the study of animal learning not solely as a simple model of human behavior, but rather as a means of searching for a broader theoretical understanding of learning.

Some commentators argued that psychologists are really interested in the mechanisms of learning, not in the way those mechanisms are employed in nature. Kalat and Shettleworth expressed that point of view quite clearly, and Garcia, Hinde, and Revusky implied it. My arguments against the cogency of that argument will be found under topic 2, above.

9. Describing the environment. In contrast to the traditional approaches, the ecological approach requires that a description of the animal's natural environment figure prominently in the study of learning. That requirement raises important conceptual and empirical problems, and I am pleased that Charlesworth, Petrinovich, and Schleidt recognize the significance of those problems. Schleidt's reference to von Uexkull's concept of the Umwelt is especially appropriate, and I agree with his suggestion that refinement of the concept is a promising course to pursue. J. J. Gibson's concept of affordance (1977) illustrates what might be done in the way of such refinement. Kruse \& Reed provide an outline of affordance theory and give references to the pertinent literature (see also Johnston \& Turvey 1980). Alley \& Shaw's comments are also in the Gibsonian tradition, and emphasize the importance of seeking animal-relevant descriptions of the environment, that is, descriptions that are given with respect to the particular developmental characteristics of the species of interest.

Detailed, sophisticated environmental descriptions are the key to discovering general principles of learning that reflect environmental similarities among different animal species. Surely such similarities exist, as Plotkin \& Odling-Smee and Rozin suggest, and investigators concerned with the ecological study of learning must devise ways to describe them. It is not unreasonable to suppose that, as a result of convergent evolution, more general principles of learning will hold among animals that occupy broadly similar niches than among animals that have widely different lifestyles. I do not agree with Malone that such groups of species are oddly defined. There are many well-known evolutionary convergences in morphological and behavioral traits among phylogenetically unrelated but ecologically similar species, and it is quite likely that the same will be true in the case of learning.

10. Learning and development. The relation between learning and behavioral development is obviously close. Both involve changes in behavior over the course of an individual's lifetime, and both emphasize the role of experience in promoting such change. There are perhaps some kinds of behavior (like food selection) that seem best described as being learned, and other kinds (like locomotion) that seem best described as being developed. However, for me, most behavior does not fit neatly into either category. Indeed, I think that it would be a good idea to try to find some way of transcending this particular dichotomy. The distinction between behavior that is learned and behavior that develops is very similar to the distinction between learning and maturation, which is unpleasantly close to the old dichotomy between learned and innate behavior. Surely no one wants to resurrect that argument. Those who would distinguish sharply between learning and development will need to be wary of the unhelpful intellectual baggage that seems fated to go along with the distinction.

Although Plotkin \& Odling-Smee call for a unified approach to the study of adaptation, they think that a distinction between learning and development is worth preserving. However, the value of that distinction can really only be assessed in relation to specific instances, and Plotkin \& Odling-Smee do not discuss enough instances in sufficient depth to determine whether the distinction is indeed worthwhile. They refer to an "accepted sense" of learning, by which I think they may mean conditioning, but it seems unwise to prejudge the learning-development issue by making learning synonymous with the nondevelopmental concept of conditioning. There are certainly a variety of mechanisms, of which conditioning is probably one, that underly normal patterns of behavioral change, and my suggestion that the hard-and-fast distinction between learning and development should be moderated is an attempt to foster a more unified study of their operation. I agree with Schleidt's comments on this point, although I would not insist, as he does, that only adaptive behavioral changes are of interest to an ecological theory of learning. The emphasis in the approach that I advocate is on whether the change occurs naturally, not on whether it is adaptive when it does occur.

Rachlin also objects to my position on the learningdevelopment issue, although his reasons are not at all clear to me. He seems to imply that it must somehow be pointless to attempt to understand how learning is involved in the development of species-typical behavior. I confess that I do not understand the basis for that position. Kruse \& Reed, on the other hand, are much more sympathetic to my position. They suggest that learning is, in fact, a form of perceptual development. in the course of which an animal becomes able to detect the affordances of its environment. That is an interesting suggestion, and one that might be further elaborated as a conceptual paradigm for the ecological study of learning.

11. Summary: Ecological thinking in the behavioral sciences. The commentators on my article can be divided into two groups: One group accepts the view that ecological considerations must be given an impor- 
tant place in the study of animal learning, although those who hold that view differ in their evaluations of my contribution to that end. The other group denies that ecology has any useful role to play in the study of learning, except perhaps as a peripheral concern for those whose interests lead them to study forms of learning that resemble those typically found in nature. It should be emphasized that these two opinions are not merely differences in research interest or in preferred methodology (although they may certainly subsume such differences). Affirming rather than denying the importance of ecological considerations is not equivalent to expressing an interest in, say, territoriality rather than escape conditioning, or a preference for field studies over laboratory experiments. The latter would be individual decisions of no real theoretical concern. But science does not progress simply because individual scientists follow their personal interests in an uncoordinated manner; rather, it progresses because those scientists harness their interests to a broadly accepted set of theoretical problems and issues - what Thomas Kuhn (1962) called a paradigm. The ecological approach is, I think, such a paradigm, although at present it is in a somewhat undeveloped state, and it differs from other paradigms in the field in the theoretical issues that it identifies as crucial for the study of learning. Central among those issues, of course, is the relationship between an animal's learning abilities and its natural environment. That relationship is seen as the fulcrum on which the analysis of learning must turn.

Several commentators pointed out the close similarity between the ecological approach to learning and the ethological approach that has been so productive in the study of other behavioral problems. But it was also pointed out (by Charlesworth and Kalat for example) that ecological thinking has often played an important role in psychology as well. Especially significant ecological contributions to psychology have been made by Barker and Wright (1955) in the field of social psychology, by Brunswik (1952) and Gibson (1966) in perception, and by Schneirla (1971) in comparative psychology. Indeed, there are definite signs that ecological thinking is becoming more and more important in many fields of psychology. That trend is exemplified by the many recent articles in American Psychologist (Miller 1977; Bronfenbenner 1977; Gibbs 1979; Petrinovich 1979), as well as by the volumes that Charlesworth cites in his commentary. Two leading spokesmen for the relatively new field of cognitive science, a productive and rapidly-moving synthesis of cognitive psychology and artificial intelligence, have recently stressed the need for a deeper concern in that field with the problems posed by the natural environment and with the way that intelligence tackles those problems (Norman 1980; Simon 1980; see also Neisser 1976).

It seems that there is reason to hope that ecological thinking may someday occupy as central a position in psychological theory as it does in biology. The study of animal learning is well-placed, by virtue of its subject matter and the concerns of at least some current practitioners, to play a decisive role in maintaining and directing that trend.

\section{References}

Alcock, J. (1979) Animal behavior: An evolutionary approach. Sunderland, Mass.: Sinauer. [TDJ]

Argyris, C. (1975) Dangers in applying results from experimental social psychology. American Psychologist 30:469-85. [LP]

Barker, R. B. \& Wright, H. F. (1955) Midwest and its children: The psychological ecology of an American town. New York: Harper \& Row. [TDJ]

Bateson, P. P. G. (1973) Internal influences on early learning in birds. In: Constraints on Learning, ed. R.A. Hinde \& J. S. Hinde, pp 101-16. London: Academic Press. [PB]

Baum, W. M. (1973a) The correlation-based law of effect. Journal of the Experimental Analysis of Behavior 20:137-53. [JAN]

(1973b) Time allocation and negative reinforcement. Journal of the Experimental Analysis of Behavior 20:313 22. [JAN]

Bekoff, M. \& Fox, M. W. (1972) Postnatal neural ontogeny: Environment-dependent and/or environment-expectant? Developmental Psychobiology 5:323-41. [TDJ]

Bitterman, M. E. (1975) The comparative analysis of learning. Science 188:699-709. [RCG, TDJ]

(1976) Flavor aversion studies. Science 192:266-67. [AWL]

(1976) Issues in the comparative psychology of learning. In: Evolution of brain and behavior in vertebrates, ed. R. B. Masterton, M. E. Bitterman, C. B. G. Campbell \& N. Hotton, pp. 217-25. Hillsdale, N. J.: Erlbaum. [TDJ]

\& Woodard, W. T. (1976) Vertebrate learning: Common processes. In: Evolution of brain and behavior in vertebrates, ed. R. B. Masterton, M. E. Bitterman, C. B. G. Campbell \& N. Hotton, pp. 169-89. Hillsdale, N. J.: Erlbaum. [TDJ]

; LoLordo, V. M.; Overmier, J. B. \& Rashotte, M. E., eds. (1979) Animal learning: Survey and analysis. New York: Plenum Press. [TDJ]

Bock, W. J. (1959) Preadaptation and multiple evolutionary pathways. Evolution 13:194-211. [TDJ]

(1980) The definition and recognition of biological adaptation. American Zoologist 20:217-27. [TDJ]

Bock, W. J. \& von Wahlert, G. (1965) Adaptation and the form-function complex. Evolution 19:269-99. [TDJ]

Bolles, R. C. (1970) Species-specific defense reactions and avoidance learning. Psychological Review 77:32-48. [TDJ, AWL, JAN]

(1971) Species-specific defense reactions. In: Aversive conditioning and learning, ed. F. R. Brush, pp. 183-233. New York: Academic Press. [TDJ]

(1972) The avoidance learning problem. In: The psychology of learning and motivation, vol. 6, ed. G. H. Bower, pp. 97-145. New York: Academic Press. [TDJ]

Brainerd, C. J. (1978) The stage question in cognitive-developmental theory. The Behavioral and Brain Sciences 1:173-213. [WRC]

Brandon, R. N. (1978) Adaptation and evolutionary theory. Studies in History and Philosophy of Science 9:181 206. [TDJ]

Breitmeyer, B. G. (1980) Unmasking visual masking: A look at the - why - behind the veil of the "how." Psychological Review 87:52-69. [TDJ]

Breland, K. \& Breland, M. (1961) The misbehavior of organisms. American Psychologist 16:681-84. [TDJ]

Bronfenbrenner, U. (1977) Toward an experimental ecology of human development. American Psychologist 32:513-531. [TDJ]

(1979) The ecology of human development: Experiments by nature and design. Cambridge, Mass.: Harvard University Press. [WRC]

Brower, L. P.; Cook, L. M. \& Croze, H. J. (1967) Predator responses to artificial Batesian mimics released in a neotropical environment. Evolution 21:11-23. [TDJ]

Brown, J. L. (1975) The evolution of behavior. New York: Norton. [TDJ]

Brown, P. L. \& Jenkins, H. M. (1968) Auto-shaping of the pigeon's key-peck. Journal of the Experimental Analysis of Behavior 11:1 -8. [AWL]

Brunswik, E. (1952) The conceptual framework of psychology. Chicago: University of Chicago Press. [MTG, LP, TDJ]

Burtt, E. H., Jr. (1979) The behavioral significance of color. New York and London: Garland STPM Press. [JPH]

Cabanac, M. (1974) Thermoregulatory behavior. In: Environmental physiology, ed. D. Robertshaw, pp. 231-69. London: Butterworths. [TDJ]

Campbell, D. T. (1974) Evolutionary epistemology. In: The philosophy of Karl Popper, vol. 1, ed. P. A. Schlipp, pp. 413-63. LaSalle, Ill.: Open Court Press. [TDJ]

Charlesworth, W. R. (1976) Human intelligence as adaptation: An ethological approach. In: The nature of intelligence, ed. L. Resnick, pp. 147-68. Hillsdale, N. J.: Erlbaum. [TDJ]

(1978) Ethology: Understanding the other half of intelligence. Social Science Information 17:231-77. [TDJ] 
(1979) An ethological approach to studying intelligence. Human Development 22:212-16. [TDJ]

Clark, R. W. (1971) Einstein: The life and times. New York: World Publishing Co. [TRA]

Clarke, J. C.; Westbrook, R. F. \& Irwin, J. (1979) Potentiation instead of overshadowing in the pigeon. Behavioral and Neural Biology 25:18-29. [SR]

Clutton-Brock, T. H. \& Harvey, P. H. (1979) Comparison and adaptation. Proceedings of the Royal Society of London, Series B 205:547-65. [TDJ]

Collias, N. E. \& Collias, E. C. (1956) Some mechanisms of family integration in ducks. Auk 73:378-400. [TDJ]

Cook, J. 0. (1953) A gradient theory of multiple-choice learning. Psychological Review 60:15-22. [JWK]

Curio, E. (1976) The ethology of predation. Berlin: Springer-Verlag. [ACK]

Darwin, C. R. (1859) On the origin of species by means of natural selection, or the preservation of favoured races in the struggle for life. London: John Murray. [TDJ]

(1876) The movements and habits of climbing plants. 2nd ed. New York: D. Appleton. [MTG]

Dennett, D. C. (1978) Beliefs about beliefs. Behavioral and Brain Sciences 1:568-70. [TDJ]

de Villiers, P. A. (1977) Choice in concurrent schedules and a quantitative formulation of the law of effect. In: Handbook of operant behavior, ed. W. K. Honig \& E. R. Staddon, pp. 233-87. Englewood Cliffs, N.J.: Prentice-Hall. [AWL, JAN]

Dobzhansky, Th. (1956) What is an adaptive trait? American Naturalist 90:337-347. [TDJ]

(1968) On some fundamental concepts of Darwinian biology. In: Evolutionary biology, vol. 2, ed. Th. Dobzhansky, M. K. Hecht \& W. M. Steere, pp. 1-34. New York: Appleton. [TDJ]

Emlen, J. M. (1973) Ecology: An evolutionary approach. Reading, Mass.: Addison-Wesley. [TDJ]

Emlen, S. T. (1967a) Migratory orientation in the indigo bunting, Passerina cyanea. Part I: Evidence for the use of celestial cues. Auk 84:30942. [TDJ]

(1967b) Migratory orientation in the indigo bunting, Passerina cyanea. Part II: Mechanism of migratory orientation. Auk 84:463-89. [TDJ]

(1969) The development of migratory orientation in young indigo buntings. Living Bird 8:113-26. [TDJ]

(1970) Celestial rotation: Its importance in the development of migratory orientation. Science 170:1198-1201. [TDJ]

(1972) The ontogenetic development of orientation capabilities. In: Animal orientation and navigation, ed. S. R. Galler, K. Schmidt-Koenig, G. J. Jacobs \& R. F. Belleville, pp. 191-210. Washington, D.C.: NASA (SP262). [TDJ, JLL]

(1975) Migration: Orientation and navigation. In: Avian biology, vol. 5, ed. D. S. Farner \& J. R. King, pp. 129-219. New York: Academic Press [TDJ, JLL, HCP]

Emlen, S. T. \& Oring, L. W. (1977) Ecology, sexual selection and the evolution of mating systems. Science 197: 215-23. [PB]

Galef, B. G. \& Osborne, B. (1978) Novel taste facilitation of the association of visual cues with toxicosis in rats. Journal of Comparative and Physiological Psychology 92:907-16. [MTG, TDJ, SR]

Garcia, J., Ervin, F. R. \& Koelling, R. A. (1966) Learning with prolonged delay of reinforcement. Psychonomic Science 5:121-22. [TDJ]

Garcia, J. \& Ervin, F. R. (1968) Gustatory-visceral and telereceptor-cutaneous conditioning - adaptations in internal and external milieus. Communica tions in Behavioral Biology Part A, 1:389-415. [JG]

Garcia, J. \& Koelling, R. A. (1966) Relation of cue to consequence in avoidance learning. Psychonomic Science 4:123-24. [JG, TDJ]

Garcia, J.; McGowan, B. K. \& Green, K. F. (1969) Biological constraints on conditioning. In: Classical Conditioning II: Current research and theory, ed. A. H. Black \& W. F. Prokasy, Hamilton, Ontario: McMaster University Press. [JG]

Garcia, J. \& Hankins, W. G. (1975) The evolution of bitter and the acquisition of toxiphobia. In: Olfaction and taste V, ed. D. A. Denton \& J. P. GoghIan, pp. 39-45. New York: Academic Press. FIG]

Garcia, J.; Palmerino, C. C.; Rusiniak, K. W. \& Kiefer, S. W. (1980, in press) Taste aversions and the nurture of instinct. In: The Neurobiology of Learning and Memory, ed. J. L. McGaugh \& R. F. Thompson. New York: Plenum Publishing Corporation. [JG]

Garcia, J. \& Rusiniak, K. W. (1980) What the nose learns from the mouth. In: Chemical Signals, ed. D. Muller-Schwarze \& R. M. Silverstein, pp. 14156. New York: Plenum Publishing Corporation. [JG, SR]

Ghiselin, M. T. (1966) On semantic pitfalls of biological adaptation. Philosophy of Science 33:147-53. [MTG]

(1974) The economy of nature and the evolution of sex. Berkeley: University of California Press. [TDJ]

Gibbon, J.; Berryman, R. \& Thompson, R. L. (1974) Contingency spaces and measures in classical and instrumental conditioning. Journal of the Exper- imental Analysis of Behavior 21:585-605. [JAN]

Gibbs, J. A. (1979) The meaning of ecologically oriented inquiry in contemporary psychology. American Psychologist 34:127-40. [TDJ]

Gibson, J. J. (1966) The senses considered as perceptual systems. Boston: Houghton-Mifflin. [TDJ, JK]

(1977) The theory of affordances. In: Perceiving, acting and knowing: To wards an ecological psychology, ed. R. Shaw \& J. Branford, pp. 67-82. Hillsdale, N.J.: Erlbaum. [TDJ, JK]

(1979) The ecological approach to visual perception. Boston: Houghton Mifflin. [WRC, JK]

Glassman, R. B. (1977) How can so little brain hold so much knowledge? Applicability of the principle of natural selection to mental processes. Psychological Record 27:393-415. [TDJ]

Gonzalez, R. C.; Ferry, M. \& Powers, A. S. (1974) The adjustment of goldfish to reduction in magnitude of reward in massed trials. Animal Learning and Behavior 2:23-26. [RCG]

Gonzalez, R. C., Potts, A., Pitcoff, K. \& Bitterman, M. E. (1972) Runway performance of goldfish as a function of complete and incomplete reduction in amount of reward. Psychonomic Science 27:305-07. [RCG]

Goss-Custard, J. D. (1980) Feeding behavior of Redshank, Tringa totanus, and optimal foraging theory. In: Foraging behavior: Ecological, ethological, and psychological approaches, ed. A. C. Kamil \& T. D. Sargent, pp. 11533. New York: Garland Publishing Inc. [ACK]

Gottlieb, G. (1971) Development of species identification in birds. Chicago: University of Chicago Press. [TDJ]

(1976) The roles of experience in the development of behavior and the nervous system. In: Neural and behavioral specificity, ed. G. Gottlieb, pp. 25-54. New York: Academic Press. [TDJ]

(1978) Development of species identification in ducklings: IV. Change in species-specific perception caused by auditory deprivation. Journal of Comparative and Physiological Psychology 92:375-87. [TDJ, SJS]

(1979) Development of species identification in ducklings: V. Perceptual differentiation in the embryo. Journal of Comparative and Physiological Psychology 93:831-54. [TDJ]

(1980) Development of species identification in ducklings: VI. Specific experience required to maintain species-typical perception in Peking ducklings. Journal of Comparative and Physiological Psychology 94:57987. [TDJ]

Gould, S. J. (1977) Ontogeny and phylogeny. Cambridge: Harvard University Press. [TRA]

(1978) Ever since Darwin. New York: Norton. [TDJ]

(1980) The evolutionary biology of constraint. Daedalus 109(2):39-52. [TDJ]

Gould, S. J. \& Lewontin, R. C. (1979) The spandrels of San Marco and the Panglossian paradigm: A critique of the adaptationist program. Proceedings of the Royal Society of London, Series B 205:581 -98. [GWB, TDB

Gray, L. \& Tardif, R. R. (1979) The development of feeding diversity in deermice. Journal of Comparative and Physiological Psychology 93:112735. [TDJ]

Hailman, J. P. (1967) The ontogeny of an instinct: The pecking response in chicks of the laughing gull (Larus atricilla L.) and related species. Behav iour Supplement 15:1-159. [JPH, TDJ]

(1969) How an instinct is learned. Scientific American 221:98-106. [RCG] (1976) Uses of the comparative study of behavior. In: Evolution, brain, and behavior: Persistent problems, ed. R. B. Masterton, W. Hodos \& J. Jenson, pp. 13-22. Hillsdale, N.J.: Erlbaum. [JPH]

(1977a) Bee dancing and evolutionary epistemology. American Naturalist, 111:187-89. [JPH]

(1977b) Optical signals: Animal communication and light. Bloomington and London: Indiana University Press. [JPH]

(1979) Environmental light and conspicuous colors. In: The behavioral significance of color, ed. E. H. Burtt, Jr., pp. 289-359. New York and London: Garland STPM Press. [JPH]

Hanson, N. (1969) Perception and discovery. An introduction to scientific inquiry. San Francisco: Freeman. [TDJ]

Herrnstein, R. J. (1970) On the law of effect. Journal of the Experimental Analysis of Behavior 13:243-66. [JAN]

Herrnstein, R. J. \& Hineline, P. N. (1966) Negative reinforcement as shock frequency reduction. Journal of the Experimental Analysis of Behavior 9:421-30. [JAN]

Hinde, R. A. (1973) Constraints on learning - an introduction to the problems In: Constraints on learning: Limitations and predispositions, ed. R. A. Hinde \& J. Stevenson-Hinde, pp. 1-19. New York: Academic Press. [P1, TDJ]

(1975) The concept of function. In: Function and evolution in behavior: Essays in honor of Professor Niko Tinbergen, FRS, ed. G. Baerends, C. G. Beer \& A. Manning, pp. 3-15. Oxford: Clarendon Press. [TDJ]

Hoffman, H. S. \& Ratner, A. (1973) A reinforcement model of imprinting: Inn plications for socialization in monkeys and man. Psychological Review 
80:527-544. [RCG]

Hogan, J. A. (1977) The ontogeny of food preferences in chicks and other animals. In: Learning mechanisms in food selection, ed. L. M. Baker, M. Best \& M. Domjan, pp. 71-97. Waco, Tex.: Baylor University Press. [TDJ]

Hollis, K. L. (in press) Pavlovian conditioning of signal-centered action patterns and autonomic behavior: A biological analysis of function. Advances in the Study of Behavior. [SJS]

Hovland, C. I. (1951) Human learning and retention. In: Handbook of experimental psychology, ed. S. S. Stevens, pp. 613-89. New York: John Wiley \& Sons. [WMS]

Hull, C. L. (1929) A functional interpretation of the conditioned reflex. Psychological Review 36:498_511. [TDJ]

(1937) Mind, mechanism and adaptive behavior. Psychological Review 44:1-32. [TDJ]

(1945) The place of innate individual and species differences in a naturalscience theory of behavior. Psychological Review 52:55-60. [MTG]

Hutchinson, G. E. (1957) Concluding remarks. Cold Spring Harbor Symposia on Quantitative Biology, 22:415_27. [WMS]

(1967) The ecological theater and the evolutionary play. New Haven, Conn.: Yale University Press. [TDJ]

(1978) An introduction to population ecology. New Haven, Conn.: Yale University Press. [JK]

Immelmann, K. (1975) Ecological significance of imprinting and early learning. Annual Review of Ecology and Systematics 6:15_37. [WRC]

Jacobson, M. (1970) Developmental neurobiology. New York: Holt. [TDJ]

Jeffords, M. R.; Sternburg, J. G. \& Waldbauer, G. P. (1979) Batesian mimicry: Field demonstration of the survival value of pipevine swallowtail and monarch color patterns. Evolution 33:275-86. [TDJ]

Jenkins, H. M. (1979) Animal learning and behavior theory. In: The first century of experimental psychology, ed. E. Hearst, pp. 177-228. Hillsdale, N.J.: Erlbaum. [TDJ]

Johnson, C.; Beaton, R. \& Hall, K. (1975) Poison-based avoidance learning in non-human primates: Use of visual cues. Physiology and Behavior 14:40307. [TDJ]

Johnston, T. D. (in press) Selective costs and benefits in the evolution of learning. In: Advances in the study of behavior, vol. 13, ed. J. S. Rosenblatt, R. A. Hinde, C. G. Beer \& M.-C. Busnel. New York: Academic Press. [TDJ]

(in preparation) "Learning" and "development" as concepts in the study of behavioral change. To appear in: Readings and essays in evolutionary epistemology, ed. H. C. Plotkin. London: John Wiley \& Sons. [TDJ]

Johnston, T. D. \& Turvey, M. T. (1980) An ecological metatheory for theories of learning. In: The psychology of learning and motivation, vol. 14, ed. G. H. Bower, pp. 147-205. New York: Academic Press. [TRA, TDJ]

Kamil, A. C. (1978) Systematic foraging by a nectar-feeding bird, the amakihi (Loxops virens). Journal of Comparative and Physiological Psychology 92:388-96. [TDJ]

Kamil, A. C. \& Sargent, T. D. eds. (1980) Foraging behavior: Ecological, ethological and psychological approaches. New York: Garland Publishing Inc. [ACK]

Kamil, A. C.; Peters, J. \& Lindstrom, F. (in press) An ecological perspective on the study of the allocation of behavior. In: Quantitative analyses of behavior II. Matching and Maximizing Accounts, ed. M. L. Commons, R. J. Hermstein \& H. Rachlin. New York: Ballinger. [ACK]

Kamin, L. J. (1968) - Attention-like-processes in classical conditioning. In: Miami symposium on the prediction of behavior: Aversive stimulation, ed. M. R. Jones, pp. 9-31. Miami: University of Miami Press. [TDJ]

(1969) Predictability, surprise, attention, and conditioning. In: Punishment and aversive behavior, ed. B. A. Campbell \& R. M. Church, pp. 279-96. New York: Appleton-Century-Crofts. [TDJ]

Kantor, J. R. (1971) The aim and progress of psychology and other sciences. Chicago: Principia Press. [JCM]

Kimble, G. A. (1973) Scientific psychology in transition. In: Contemporary approaches to learning and memory, ed. F. J. McGuigan \& D. B. Lumsden, pp. 1-19. Washington, D.S.: V. H. Winston \& Sons. [TDJ]

Krebs, J. R. (1978) Optimal foraging: Decision rules for predators. In: Bahavioural ecology, ed. J. R. Krebs \& N. B. Davies, pp. 23-63. Oxford: Blackwell. [ACK]

Krebs, J. R.; Erichsen, J. T.; Webber, M. I. \& Charnov, E. L. (1977) Optimal prey selection in the great tit (Parus major). Animal Behaviour 25:3038. [ACK]

Kohler, Wolfgang (1925) The mentality of apes. London: Routledge \& K. Paul. (Translated from the German.) [JK]

Kuhn, T. S. (1962) The structure of scientific revolutions. Chicago: University of Chicago Press. [TDJ]

Kuo, Z. Y. (1922) How are our instincts acquired? Psychological Review 29:344-65. [TDJ]

(1929) The net result of the anti-heredity movement in psychology. Psycho- logical Review 36:181-99. [TDJ]

(1967) The dynamics of behavior development. New York: Random House. [TDJ]

Lachman, J. L. \& Lachman, R. (1979) Theories of memory organization and human evolution. In: Memory organization and structure, ed. R. C. Puff, pp. 133-193. New York: Academic Press. [TDJ, JLL]

(1980) Picture-naming: Retrieval and activation of long-term memory. In: New directions in memory and aging: Proceedings of the George Tolland Memorial Conference, ed. L. W. Poon, J. L. Fozard, L. S. Cermak, D. Arenberg, and L. W. Thompson, pp. 313-43. [JLL]

Lachman, R.; Lachman, J. L. \& Butterfield, E. C. (1979) Cognitive psychology and information processing: An introduction. Hillsdale, N.J.: Erlbaum. [JLL]

Lakatos, 1. (1970) Falsification and the methodology of scientific research programmes. In: Criticism and the growth of knowledge, ed. I. Lakatos \& A Musgrave, pp. 91-195. Cambridge: Cambridge University Press. [TDJ]

Lattal, K. A. (1974) Combinations of response-reinforcer dependence and independence. Journal of the Experimental Analysis of Behaviour 22:35762. [JAN]

Lea, S. E. G. (1979) Foraging and reinforcement schedules in the pigeon: Optimal and non-optimal aspects of choice. Animal Behavior 27:821828. [ACK, SJS]

Lehrman, D. S. (1953) A critique of Konrad Lorenz's theory of instinct. Quarterly Review of Biology 28:337 -63. [TDJ]

(1962) Varieties of learning and memory in animals. In: Macromolecular specificity and biological memory, ed. F. 0. Schmitt, pp. 108-10. Cambridge, Mass.: MIT Press. [TDJ]

(1970) Semantic and conceptual issues in the nature-nurture problem. In: Development and evolution of behavior. Essays in memory of T. C. Schneirla, ed. L. R. Aronson, E. Tobach, D. S. Lehrman \& J. S. Rosenblatt, pp. 17-52. San Francisco: Freeman. [TDJ, SJS]

Leigh, E. G. (1971) Adaptation and diversity. San Francisco: Freeman. [TRA]

Lett, B. T. (1980) Taste potentiates color-sickness associations in pigeons and quail. Animal Learning and Behavior 8:193-98. [AWL, SR]

Lewontin, R. C. (1978) Adaptation. Scientific American 239:212-30. [TRA, TDJ]

(1979) Sociobiology as an adaptationist program. Behavioral Science 24:5-14. [TDJ, BS]

Lockard, R. B. (1971) Reflections on the rise and fall of comparative psychology: Is there a message for us all? American Psychologist 26:168-179. [ACK]

Logue, A. W. (1978) John B. Watson and the continuity of the species. Behaviorism 6:71-79. [AWL]

(1979) Taste aversion and the generality of the laws of learning. Psychological Bulletin 86:276-296. [TDS, AWL]

(1980) Visual cues for illness-induced aversions in the pigeon. Behavioral and Neural Biology 28:372 -77. [AWL]

(in press a) The origins of behaviorism. In: A history of modern psychology: Concepts, methods, viewpoints, ed. C. Buxton. New York: Academic Press. [AWL]

(in press b) The growth of behaviorism. In: A history of modern psychology: Concepts, methods, viewpoints, ed. C. Buxton. New York: Academic Press. [AWL]

(in press c) The generality of taste aversion learning in humans. In: Quantitative analyses of behavior: Acquisition, ed. M. L. Commons, A. R. Wagner \& R. J. Herrnstein. Cambridge, Mass. Ballinger Publishing Co. [AWL]

LoLordo, V. M. (1979) Constraints on learning. In: Animal learning: Survey and analysis, ed. M. E. Bitterman, V. M. LoLordo, J. B. Overmier \& M. E. Rashotte, pp. 473-504. New York: Plenum Press. [TDJ]

Lorenz, K. (1941) Kant's doctrine of the a priori in the light of contemporary biology. General Systems, vol. VII. Ann Arbor: University of Michigan Press, 1962, pp. 23-35. (Originally published in Blatter für Deutsche Philosophie, 1941, 15:94-125.) [WRC]

(1965) Evolution and modification of behavior. Chicago: University of Chicago Press. [TDJ, WMS]

(1969) Innate bases of learning. In: On the biology of learning, ed. K. H. Pribram, pp. 13-93. New York: Harcourt, Brace \& World. [TDJ]

(1977) Behind the mirror: A search for a natural history of human knowledge. New York: Harcourt Brace Jovanovich. [WRC]

Lynch, K. (1961) The image of the city. Cambridge, Mass.: MIT Press. [TDJ]

MacArthur, R. H. \& Pianka, E. R. (1966) On optimal use of a patchy environment. American Naturalist 100:603-09. [ACK]

MacArthur, R. W. \& Wilson, E. 0. (1967) The theory of island biogeography. Princeton, N.J.: Princeton University Press. [TRA]

McCall, R. B. (1977) Challenges to a science of developmental psychology. Child Development 48:333_44. [TDJ]

McGurk, H., ed. (1977) Ecological factors in human development. New 
York: North Holland. [WRC]

McNamara, J. \& Houston, A. (1980) The application of statistical decision theory to animal behavior. Journal of Theoretical Biology 85:673-90. [SJS]

Maier, N. R. F. \& Schneirla, T. C. (1935) Principles of animal psychology. New York: McGraw-Hill. [JWK]

Malone, J. C., Jr. (1973) A note on general process learning theorists. Psychological Review 80:305. [JCM]

(1975) The "paradigms" of learning. Psychological Record 25:479-

89. [TDJ, JCM]

(1978) Beyond the operant analysis of behavior. Behavior Therapy 9:58491. [JCM]

Mader, P. R. (1970) A comparative approach to vocal development: Song learning in the white-crowned sparrow. Journal of Comparative and Physiological Psychology Monographs 71 (No. 2, Pt. 2):1 - 25. [TDJ, JAN]

(1977) Perception and innate knowledge. In: The nature of life, ed. W. H. Heidcamp, pp. 111-39. Baltimore: University Park Press. [TDJ]

Marler, P. R. \& Mundinger, P. (1971) Vocal learning in birds. In: Ontogeny of vertebrate behavior, ed. H. Moltz, pp. 389-450. New York: Academic Press. [TDJ, SJS]

Mason, H. L. \& Langenheim, J. H. (1957) Language analysis and the concept environment. Ecology 38:325_40. [TDJ]

Mayr, E. (1963) Animal species and evolution. Cambridge, Mass.: Belknap Press of Harvard University Press. [TDJ]

(1974) Behavior programs and evolutionary strategies. American Scientist 62:650-59. [TRA, WRC, HP]

(1974) Teleological and teleonomic: A new analysis. Boston Studies in the Philosophy of Science 14:91 - 117. [LP]

Medawar, P. B. (1969) Induction and intuition in scientific thought. Philadel phia: American Philosophical Society. [TM]

Miller, D. B. (1977) Roles of naturalistic observation in comparative psychology. American Psychologist 32:211 - 19. [TDJ]

Miller, D. B. \& Gottlieb, G. (1978) Maternal vocalizations of mallard ducks (Anas platyrhynchos). Animal Behaviour 26:1178_94. [TDJ]

Mills, W. (1898) The nature and development of animal intelligence. London: T. Fisher Unwin. [WRC]

Moore, B. R. (1973) The role of directed Pavlovian reactions in simple instrumental learning in the pigeon. In: R. A. Hinde and J. Stevenson-Hinde (eds.), Constraints on Learning. London: Academic Press. [SR]

Neisser, U. (1976) Cognition and reality. San Francisco: Freeman. [TDJ]

Nevin, J. A. (1974) Response strength in multiple schedules. Journal of the Ex perimental Analysis of Behavior 21:389_408. [JAN]

(1979) Reinforcement schedules and response strength. In: Reinforcement and the organization of behavior, ed. M. Zeiler \& P. Harzem. Chichester, England: Wiley. [JAN]

Norman, D. A. (1980) Twelve issues for cognitive science. Cognitive Science 4:1-32. [TDJ]

Nottebohm, F. (1970) Ontogeny of bird song. Science 167:950-56. [TDJ]

Novikoff, A. B. (1945) The concept of integrative levels and biology. Science 101:209-15. [WMS]

Odum, E. P. (1959) Fundamentals of ecology. Philadelphia: W. B. Saunders. [WMS]

Oppenheim, R. W. (in press) Preformation and epigenesis in the origins of the nervous system and behavior: Issues, concepts and their history. In: Per spectives in ethology, vol. 5, ed. P. P. G. Bateson \& P. H. Klopfer. New York: Plenum Press. [TDJ]

Orians, G. H. (1969) On the evolution of mating systems in birds and mammals. American Naturalist 103:589-603. [PB]

Pantin, C. F. A. (1951) Organic design. Advances in Science 30:1-13. [RAH] (1952) The elementary nervous system. Proceedings of the Royal Society B. 140:147-68. [RAH]

Pavlov, I. P. (1927) Conditioned reflexes, tr. G. V. Anrep. London: Oxford University Press. [JCM]

(1932) The reply of a physiologist to psychologists. Psychological Review 39:91-127. [JCM]

Pert, A. \& Gonzalez, R. C. (1974) Behavior of the turtle (Chrysemys Picta Picta) in simultaneous, successive and behavioral contrast situations. Jour nal of Comparative and Physiological Psychology 87:526_38. [RCG]

Petrinovich, L. (1973) Darwin and the representative expression of reality. In: Darwin and facial expression, ed. P. Ekman, pp. 223-56. New York: Academic Press. [LP]

(1976) Molar reductionism. In: Knowing, thinking, and believing, ed. L. Petrinovich \& J. L. McGaugh, pp. 11-27. New York: Plenum Press. [LP]

(1979) Probabilistic functionalism: A conception of research method. American Psychologist 34:373-90. [TDJ, LP]

(in press) A method for the study of development. In: Interdisciplinary study of development: The Bielefeld conference, ed. K. Immelmann, G.
Barlow, M. Main \& L. Petrinovich. New York: Cambridge University Press. [LP]

Pietrewicz, A. T. \& Kamil, A. C. (1977) Visual detection of cryptic prey by blue jays (Cyanocitta cristata). Science 195:580 - 82. [TDJ]

(1979) Search image formation in the blue jay (Cyanocitta cristata). Science 204:1332-33. [TDJ]

Piaget, J. (1971) Biology and knowledge. Edinburgh: The University of Edinburgh Press. [HCP]

Plotkin, H. C. (in press) The evolution of closed and open programmes of development. In: Development in the nervous system, ed. D. R. Garrod j, I Feldman. Cambridge University Press. [HCP]

Plotkin, H. C. \& Odling-Smee, F. J. (1979) Learning, change and evolution: An enquiry into the teleonomy of learning. Advances in the Study of Behaviour 10:1-41. [HCP, SJS]

Polanyi, K. (1944) The great transformation. New York: Rinehart. [BS]

Premack, D. (1976) Intelligence in ape and man. Hillsdale, N.J.: Erlbaum. [PR]

Pyke, G. H.; Pulliam, H. R. \& Charnov, E. L. (1977) Optimal foraging: A selective review of theory and tests. Quarterly Review of Biology 52:11754. $[\mathrm{ACK}]$

Pylyshyn, Z. (1978) Computational models and empirical constraints. The Behavioral and Brain Sciences 1:93-99. [JLL]

Rachlin, H. \& Baum, W. M. (1972) Effects of alternative reinforcement: Does the source matter? Journal of the Experimental Analysis of Behavior 18:231-41. [JAN]

Reed, E. S. (1979) An evolutionary analysis of intentional activity. Masters Thesis, University of Edinburgh. [WRC]

Reed, E. S. \& Jones, R. K. (1979) James Gibson's ecological revolution in psychology. Philosophy of Social Science 9:189_204. [JK]

Rescorla, R. A. (1967) Pavlovian conditioning and its proper control procedures. Psychological Review 74:71-80. [PR]

(1972) Informational variables in Pavlovian conditioning. In: The psychology of learning and motivation, vol. 6, ed. G. H. Bower, pp. 1 -46. New York: Academic Press. [TDJ]

(1980) Pavlovian second-order conditioning. Hillsdale, N.J.: Erlbaum. [AWL]

Rescorla, R. A. \& Holland, P. C. (1976) Some behavioral approaches to the study of learning. In: Neural mechanisms of learning and memory, ed. M. R. Rosenzweig \& E. L. Bennett, pp. 165-92. Cambridge, Mass.: MIT Press. [TDJ]

Rescorla, R. A. \& Wagner, A. R. (1972) A theory of Pavlovian conditioning: Variations in the effectiveness of reinforcement and non-reinforcement In: Classical conditioning II, ed. A. H. Black \& W. F. Prokasy, pp. 64-99. New York: Appleton-Century-Crofts. [TDJ]

Revusky, S. (1971) The role of interference in association over a delay. In: Animal memory, ed. W. K. Honig \& P. H. R. James, pp. 155-213. New York: Academic Press. [PB, TDJ]

(1977) Interference with progress by the scientific establishment: Examples from flavor aversion learning, In: Food aversion learning, ed. N. W. Milgram, L. Krames \& T. M. Alloway, pp. 53-71. New York: Plenum Press. [AWL]

(1977) Learning as a general process with an emphasis on data from feeding experiments. In: Food aversion learning, ed. N. W. Milgram, L. Krames $\&$ T. M. Alloway, pp. 1-51. New York: Plenum Press. [TDJ]

Revusky, S. \& Garcia, J. (1970) Learned associations over long delays. In: The psychology of learning and motivation, vol. 4, ed. G. H. Bower \& J. T. Spence, pp. 1-84. New York: Academic Press. [TDJ, JAN]

Riley, A. L. \& Baril, L. L. (1976) Conditioned taste aversions: a bibliography. Animal Learning and Behavior 4(B):1S - 13S. [TDJ]

Rozin, P. (1976a) The evolution of intelligence and access to the cognitive unconscious. In: Progress in psychobiology and physiological psychology, vol. 6, ed. J. A. Sprague \& A. N. Epstein, pp. 245-80. New York: Academic Press. [PR]

(1976b) The selection of foods by rats, humans and other animals. In: Advances in the study of behavior, vol. 6, ed. J. Rosenblatt, R. A. Hinde, C. Beer \& E. Shaw, pp. 21-76. New York: Academic Press. [PR]

(1977) The significance of learning mechanisms in food selection: Some biol ogy, psychology and sociology of science. In: Learning mechanisms in food selection, ed. L. M. Barker, M. Best \& M. Domjan, pp. 557-89. Waco, Texas: Baylor University Press. [PR]

Rozin, P. \& Kalat, J. W. (1971) Specific hungers and poison avoidance as adap tive specializations of learning. Psychological Review 78:459-86. [TDJ, JWK, PR]

(1972) Learning as a situation-specific adaptation. In: Biological boundries of learning, ed. M. E. P. Seligman \& J. L. Hager, pp. 66-96. New York. Appleton-Century-Crofts. [PR]

Sackett, G. P., ed. (1978) Observing behavior: Theory and applications in 
mental retardation. Baltimore: University Park Press. [WRC]

Schleidt, W. M. (1981) The behavior of organisms, as it is linked to genes and populations. In: Perspectives in Ethology, ed. P. P. G. Bateson \& P. H. Klopfer, Vol. 4, pp. 147-55. New York: Plenum Publishing Corporation. [WMS]

Schleidt, W. M. \& Crawley, J. N. (1980) Patterns in the behaviour of organisms. Journal for Social and Biological Structure 3:1 - 15. [WMS]

Schneirla, T. C. (1949) Levels in the psychological capacities of animals. In: Philosophy for the Future, ed. R. W. Sellars et al. New York: Macmillan. $[\mathrm{RAH}]$

(1950) The relationship between observation and experiment in the field study of behavior. Annals of the New York Academy of Science 51:102244. [ID), JWK]

(1956) Interrelationships of the "innate" and the "acquired" in instinctive behavior. In: L'Instinct dans le comportement des animaux et de l'homme, ed. P. P. Grasse, pp. 387 -952. Paris: Masson. [TDJ] (1971) Army ants: A study in social organization. San Francisco: Freeman. [TDJ]

Schwartz, B. (1979) On going back to nature: A review of Seligman and Hager's Biological Boundaries of Learning. Journal of the Experimental Analysis of Behavior 21:183_98. [TDJ, BS]

Seligman, M. E. P. (1970) On the generality of the laws of learning. Psycholog ical Review 77:406-18. [TDJ, JCM, LP]

Seligman, M. E. P. \& Hager, J. L., eds. (1972) Biological boundaries of learning. Englewood Cliffs, N.J.: Prentice-Hall. [JG, TDJ, AWL]

Sevenster, P. (1973) Incompatibility of response and reward. In: Constraints on Learning, ed. R. A. Hinde and J. Stevenson-Hinde. Academic Press: London. [SR]

Shaw, R. \& Bransford, J., eds. (1977) Perceiving, acting, and knowing: Toward an ecological psychology. New York: John Wiley \& Sons. [WRC]

Shaw, R. E. \& Turvey, M. T. (in press) Coalitions as models for ecosystems: A realist perspective on perceptual organization. In: Perceptual organization, ed. M. Kubovy \& J. Pomerantz. Hillsdale, N.J.: Erlbaum. [TRA, JK]

Shettleworth, S. J. (1972) Constraints on learning. In: Advances in the study of behavior, vol. 4, ed. D. S. Lehrman, R. A. Hinde \& E. Shaw, pp. 1-68. New York: Academic Press. [TDJ]

(1975) Reinforcement and the organization of behavior in golden hamsters: Hunger, environment, and food reinforcement. Journal of Experimental Psychology: Animal Behavior Processes 104:56-87. [TDJ]

(in press) Function and mechanism in learning. In: Advances in analysis of behavior, vol. 3: Biological factors in learning, ed. M. Zeiler \& P. Harzem. New York: John Wiley \& Sons. [SJS]

Simon, H. A. (1969) The sciences of the artificial. Cambridge, Mass.: MIT Press. [TDJ]

(1980) Cognitive science: The newest science of the artificial. Cognitive Science 4:33-46. [TDJ]

Skinner, B. F. (1931) The concept of the reflex in the description of behavior. Journal of General Psychology 5:427-58. [JCM]

(1950) Are theories of learning necessary? Psychological Review 57:193216. [TDJ]

(1959) A case history in scientific method. In: Psychology: A study of a science, vol 2, ed. S. Koch, pp. 359-79. New York: McGraw-Hill. [JG, AWL]

(1966) The phylogeny and ontogeny of behavior. Science 153:1205-13. [JCM]

Slobodkin, L. B. (1968) Toward a predictive theory of evolution. In: Population biology and evolution, ed. R. C. Lewontin, pp. 187-205. Syracuse, N.Y.: Syracuse University Press. [TDJ]

Slobodkin, L. B. \& Rapoport, A. (1974) An optimum strategy of evolution.
Quarterly Review of Biology 49:181 - 200. [TDJ]

Small, W. S. (1901) Experimental study of the mental processes of the rat. II American Journal of Psychology 12:206-39. [TDJ, HR]

Smart, J. J. C. (1963) Philosophy and scientific realism. London: Routledge and Kegan Paul. [MTG]

Sommerhoff, G. (1950) Analytical biology. London: Oxford University Press. [TDJ]

Spence, K. W. (1947) The role of secondary reinforcement in delayed reward learning. Psychological Review 54:1-8. [JG]

Staddon, J. E. R. (1975) Learning as adaptation. In: Handbook of learning and cognitive processes, vol. 2, ed. W. K. Estes, pp. 37-98. Hillsdale, N.J.: Erlbaum. [TDJ]

Staddon, J. E. R. ed. (1980) Limits to action: The allocation of individual behavior. New York: Academic Press. [JAN]

Staddon, J. E. R. \& Simmelhag, V. L. (1971) The "superstition" experiment: A reexamination of its implications for the principles of adaptive behavior. Psychological Review 78:3_43. [TDJ]

Sutherland, N. S. \& Mackintosh, N. J. (1971) Mechanisms of animal discrimination learning. New York: Academic Press. [TDJ]

Taylor, E. F. \& Wheeler, J. A. (1966) Spacetime physics. San Francisco: Freeman. [TRA]

Thorndike, E. L. (1965) Animal intelligence. New York: Hafner. [RCG, TDJ, AWL]

Thorpe, W. H. (1963) Learning and instinct in animals, 2nd ed. London: Methuen. [TDJ]

Tinbergen, N. (1951) The study of instinct. London: Oxford University Press. [RAH, TDJ, LP]

Tinbergen, N. (1963) On aims and methods of ethology. Zeitschrift far Tierpsychologie 20:410-29. [TDJ]

Tinbergen, N.; Broekhuysen, G. K.; Feekes, F.; Houghton, J. C. W.; Kruuk, H. \& Szulc, E. (1962) Egg shell removal by the black-headed gull, Larus ridibundus, L.; A behaviour component of camouflage. Behaviour 19:74117. $[\mathrm{PB}]$

Tolman, E. C. (1938) The determiners of behavior at a choice point. Psychological Review 45:1 -41. [TDJ]

(1949) There is more than one kind of learning. Psychological Review 56:144-55. [TDJ]

Turvey, M. T. \& Shaw, R. E. (1979) The primacy of perceiving: an ecological reformulation of perception for understanding memory. In: Perspectives on memory research, ed. L.-G Nilsson, pp. 167-222. Hillsdale, N.J.: Erlbaum. [TRA, TDJ] stinctive behavior, ed. \& trans. C. H. Schiller, pp. 5-80. New York: International University Press. [WMS]

Walls, G. L. (1942) The vertebrate eye and its adaptive radiation. Bloomfield Hills, Mich.: Cranbrook Institute of Science. [TDJ, PR, SR]

Whittaker, R. H., Levin, S. A. \& Root, R. B. (1973) Niche, habitat, and ecotope. American Naturalist 107:321-38. [TDJ]

Wiens, J. A. (1970) Effects of early experience on substrate pattern selection in Rana aurora tadpoles. Copeia 1970:543-48. [TDJ]

Wigner, E. (1967) Symmetries and reflections. Cambridge, Mass.: MIT Press. [TRA]

Wilcoxon, H. C., Dragoin, W. B. \& Kral, P. A. (1971) Illness-induced aversions in rat and quail: Relative salience of visual and gustatory cues. Science 171:826-28. [TDJ, AWL]

Williams, C. G. (1966) Adaptation and natural selection. Princeton, N.J.: Princeton University Press. [TDJ]

Yarczower, M. \& Hazlett, L. (1977) Evolutionary scales and anagenesis. Psychological Bulletin 84:1088-97. [RCG] 